\title{
Comet Halley, Parameter Study I
}

W. F. Huebner

M. M. Fikani

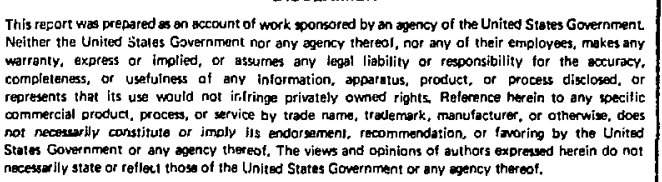

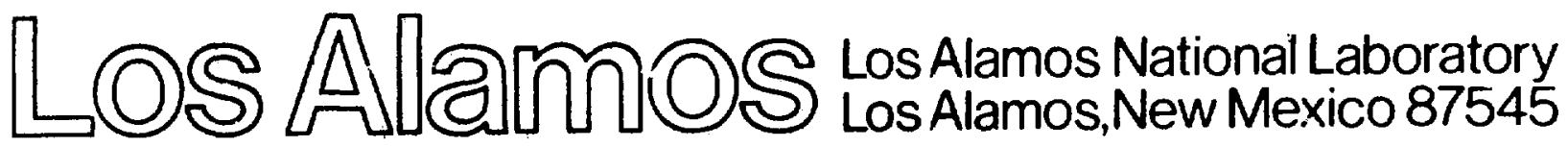


COMET HALLEY, PARAMETER STUDY I

$$
\text { by }
$$

W. F. Huebner and M. M. Fikant

\begin{abstract}
To ald In defining a mission to comet P/Halley, its Inner coma is simulated by a computer program that models time-dependent chemical reactions in a radially and isentropically expanding gas, taking tnto account attenuation of solar ultraviolet radiation in the subsolar direction. Column density predictions are based on intelligently selected combinations of poorly known values for nucleus parameters that include size, visual albedo, and Infrared emissivity. Only one chemical composition and a minor modification of 1 t are considered here; the dust-to-gas ratio in this model 18 zero. Although the somewhat optimistically volatile composition chosen here favors a smaller nucleus, a mean nuclear radius of only $0.5 \mathrm{~km} 1 \mathrm{~s}$ unlikely. No significant increase of molecular column density 18 predicted by this model as a gpacecraft approaches, once it is less than a few $10^{4} \mathrm{~km}$ from the nucleus. Predictions are made for various heliocentric distances of interest for comet missions and for ground observations.
\end{abstract}


Presented in Table I are observational data from the 1910 apparition of periodic comet Halley that are of primary interest for comparison with the model's predictions. In particular, three species $\left(\mathrm{CN}, \mathrm{C}_{2}\right.$, and $\left.\mathrm{C}_{3}\right)$ are traced through an Important segment of comet Halley's orbit -- roughly 3 AU heliocentric distance pre-perihelion to $1.4 \mathrm{AU}$ post-perihelion. Given are the physical extent in the coma of the species based on spectral images in thousands of kilometers, as well as their relative logarithmic intensity (determined by photographic densities as discussed by Bobrovnikof $f^{1}$ ). Relevant comments are included for clarity where needed along with those references that proved most valuable in making the trace. It should be borne in mind that, because of the observational quality that existed around the time of the 1910 apparition (and to purported corrections made thereafter), the values presented are not absolute. It is felt that the proper behavior is intact and it is this which is important for comparison.

General observational features include an apparent spectral change -- solar $\left(\lambda \approx 470 \AA^{\circ}\right)$ - violet $(\lambda \approx 4000 \AA)$ - occurring at approximate1y $1.2 \mathrm{AU}$. Comparison with the cometary average ${ }^{2}$ at $\sim 0.8$ AU indicates this possibly to be an important effect. However, the reality of this spectral change appears to be in some doubt. It is felt by some that; in the pre-perihelion approach (constituting a violet-to-solar change), the decrease in brightness of $\mathrm{CN}, 1 . e .$, the violet spectrum, was only apparent -- $\mathrm{CN}$ and $\mathrm{C}_{3}$ actually do increase but are overshadowed by the reflection spectrum and not by a significant increase in the $\mathrm{C}_{2} / \mathrm{CN}$ and $\mathrm{C}_{2} / \mathrm{C}_{3}$ ratios. Other $\mathrm{s}^{3}$ feel the spectral change can be attributed to "bad procedures," 1.e., poor quality of observations as well as fallacious corrections. In any event, the face value of the observations shows a spectral change that must be accounted for.

Among other observational features were noted: Na-D 11nes observed in envelopes and extending into the tall during perihelion passage; $\mathrm{CH}$ and various bands of $\mathrm{C}_{2}, \mathrm{C}_{3}$, and $\mathrm{CN}$ were positively Identified; no $\mathrm{CO}^{+}$found in envelopes (parabolic or elliptical) but definitely in tall along with $\mathrm{N}_{2}^{+}$. It should be mentioned in confunction with the last statement that both the parabolic and elliptical envelopes were essentially unfform in mixture of $\mathrm{CN}$ and $\mathrm{C}_{2}$. 
Table I

Extent $\rho\left(10^{3} \mathrm{~km}\right) /$ Intensity $[\log \mathrm{I}]$ of Spectral Images of P/Ha11ey (1910 II)

$\underline{r / d a t e} \quad \underline{C N} \quad C_{2} \quad \underline{C_{3}} \quad \underline{R e f e r e n c e s}$ and Congents

$2.95 \mathrm{AU} \quad$ Faint uniform continuum, CN and

(Oct 22, 1909)

$\sim 2.5 \mathrm{AU}$

(Dec 18,1909 )

$\sim 2.3 \mathrm{AU}$

(Dec 30, 1909)

$\sim 2.1 \mathrm{AU}$

(Jan 09, 1910)

$\sim 2.0$ AU

(Jan 15, 1910)

$1.7-1.6 \mathrm{AJ}$

(Feb 04, 1910)

$1.6 \mathrm{AU}$

(Feb 10, 1910)

$1.2-1.1 \mathrm{AU}$

(Mar 08, 1910)

$0.6 \mathrm{AU}$

(Apr 21, 1910)

$0.7 \mathrm{AU}$

(May 05, 1910)

$20.85 \mathrm{AU}$

(May 27, 1910)

$1.0 \mathrm{AU}$

(Jun 06, 1910)

$1.4 \mathrm{AU}$

(Jun 26, 1910)

$c_{2}$ suspect (faint), $C_{3}$ (4050A) observed faintly

$95.2 / 0.35$

$74.6 / 0.2$

$77.1 /-$

$135 / 0.45 \quad 97 / 0.08 \quad 64.9 /-$

$90 / 0.25$

$84.5 / 0.17$

$64.5 /-$

$65.2 / 0.4$

$57.7 / 0.3$

$42.0 /-$

$62.5 / 0.25 \quad 69 / 0.15 \quad 42.8 /-$

$69.1 / 0.05 \quad 106.6 / 0.05 \quad(35)^{\mathrm{b} /-}$

$21.2 / 0.55 \quad 40.5 / 1.0 \quad 8.0 /-$

$59.7 / 0.15 \quad 48.1 / 0.1 \quad 46.5 /-$
$71.9 / 0.25 \quad 49.5 / 0.05 \quad 49.6 /-\quad 1 /$

$1 /$

1/; note: maximum extension of $\mathrm{CN}$

$1 /$

$1 /$

$1 / 2$; $C N$ and $C_{2}$ become comparable in strength

$1 / 1$; reported spectral change;

note: $\max$ extension of $c_{2}$

$(38)^{b} / 0.65 \quad 46.1 / 0.8 \quad(20)^{b} /-$

1/; near perthelion (Apr 19, 1910)

1/1; primar1ly in reflected light, fluctuations;

note: throughout May envelopes

are at minimum extension

$15.4 / 1.0 \quad 14.3 / 1.4 \quad 9.4 /-\quad 1 /$

$23.2 / 1.3 \quad 37.3 / 1.4 \quad 16.7 /-\quad 1 /$

$1 / 1$; CN becomes dontnant, $\mathrm{C}_{3}$ and $C N$ exhibit oimilar behavior

a F1rst number 18 reference for extent; second number is reference

for intensity.

b Estimate from supplied graph in Ref. 1 . 
II. THE COMPUTER MODEL

\section{A. Physics}

The model nucleus is assumed to be a homogenous mixture of volatile frozen gases assembled into a spherical shape of radius $R_{0}$. Solar radiation, mostly in the visual range, impinges on the surface. Depending on the albedo, $A$, part of the radiation is reflected, the rest is absorbed. The infrared enlssivity, $\varepsilon$, determines the fraction of absorbed energy that is re-emitted. The rest of the absorbed energy causes sublimation (phase change from solid to gas) of the frozen gases. Because the nucleus rotates, it is assumed that energy for sublimation is distributed unfformly over the surface of the sphere.

Using the adiabatic exponent as the polytropic exponent, the supersonic von Mises ${ }^{4}$ solution describes the isentropic outflow of the coma gas. Solar ultraviolet attenuation is calculated, in wavelength bins a few to $50 \AA$ wide, from the density-distance relationshtp of the coma gas,

$$
n=n_{0} \frac{v_{0} R_{0}}{v R}
$$

and the photoelectric cross sections and abundances of the mother molecules. In Eq.(1) $n$ is the gas density and $v$ is the outstream speed in the subsolar direction at a distance $R$ from the center of the nucleus. The symbols with subscript o Indicate corresponding variables at the nucleus surface. Two-dimensional test calculations of attenuation indicate no large deviation of the chemistry from the subsolar direction except in a narrow cone in the antisolar direction. Solar ultraviolet radiation causes dissociation, ionization, and dissoclative lonlzation producing radicals and ions that initiate a complex network of chemical reactions. For more details on the physics see Refs. 5-7.

\section{B. Chenistry}

The nucleus is assumed to have composition 6 a (according to our numbering scheme, see Ref. 8). This composition is reproduced in Table II. 


\section{Intital Composition}

\begin{tabular}{lc} 
Species & Percer:t Number Abundance \\
\hline $\mathrm{H}_{2} \mathrm{O}$ & 43.0 \\
$\mathrm{H}_{2} \mathrm{CO}$ & 22.1 \\
$\mathrm{CH}_{4}$ & 13.4 \\
$\mathrm{CO}_{2}$ & 12.0 \\
$\mathrm{~N}_{2}$ & 5.2 \\
$\mathrm{CO}$ & 2.8 \\
$\mathrm{HCN}$ & 0.5 \\
$\mathrm{CH}_{3} \mathrm{CN}$ & 0.4 \\
$\mathrm{CH}_{3} \mathrm{NH}_{2}$ & 0.2 \\
$\mathrm{H}_{2} \mathrm{C}_{3} \mathrm{H}_{2}$ & 0.2 \\
$\mathrm{NH}_{3}$ & 0.1 \\
$\mathrm{C}_{2} \mathrm{H}_{2}$ & 0.1
\end{tabular}

It was pointed out by $A^{\prime}$ Hearn in a private communication ${ }^{9}$ that a recently quoted oscillator strength used by hIm and others is too low by a factor of about 40. For this reason we have revised the relative abundance of two trace molecules $\mathrm{C}_{2} \mathrm{H}_{2}$ from $0.1 \%$ to $0.16 \%$ and $\mathrm{H}_{2} \mathrm{C}_{3} \mathrm{H}_{2}$ from $0.2 \%$ to $0.005 \%$. This modified composition will be referred to as $6 \mathrm{a}^{\prime}$. These modifications do not change the relative elemental abundances (see Ref. 8).

The 1ist of species used in the time-dependent chemical kinetics is given In Table III, and the reactions and the assoclated coefflcients $A, B$, and $C$ for the Arrhenius equation are given in Table IV. The reactions and assoctated rate coefficients are a summary of those that have been complled and 1 isted In Refs. 5,6 , and 8 . The rate coefflclents for the chemlcal reactions are of the form

$$
k=A\left(\frac{T}{300}\right)^{B} e^{-C / T}
$$

For photolytic processes the rate coefficient is independent of $B$ and $C$ but has to be corrected for ultraviolet attenuation. 
Species Included in Models

Electrons and Monatomics

\begin{tabular}{lllllllll}
\hline e & $\mathrm{H}$ & $\mathrm{H}^{+}$ & $\mathrm{C}$ & $\mathrm{C}\left({ }^{1} \mathrm{D}\right)$ & $\mathrm{C}^{+}$ & $\mathrm{N}$ & $\mathrm{N}^{+}$ \\
0 & $O\left({ }^{1} \mathrm{D}\right)$ & $\mathrm{O}^{+}$ & & & & & &
\end{tabular}

\section{Diatomics}

\begin{tabular}{llllllll}
\hline $\mathrm{H}_{2}$ & $\mathrm{H}_{2}{ }^{+}$ & $\mathrm{CH}$ & $\mathrm{CH}^{+}$ & $\mathrm{NH}$ & $\mathrm{NH}^{+}$ & $\mathrm{OH}$ & $\mathrm{OH}^{+}$ \\
$\mathrm{C}_{2}$ & $\mathrm{C}_{2}{ }^{+}$ & $\mathrm{CN}$ & $\mathrm{CN}^{+}$ & $\mathrm{CO}$ & $\mathrm{CO}\left(\mathrm{a}^{3} \mathrm{II}\right)$ & $\mathrm{CO}^{+}$ & $\mathrm{N}_{2}$ \\
$\mathrm{~N}_{2}{ }^{+}$ & $\mathrm{NO}$ & $\mathrm{NO}^{+}$ & $\mathrm{O}_{2}$ & $\mathrm{O}_{2}^{+}$ & & &
\end{tabular}

Triatomics

\begin{tabular}{llllllll}
\hline $\mathrm{H}_{3}^{+}$ & $\mathrm{CH}_{2}$ & $\mathrm{CH}_{2}{ }^{+}$ & $\mathrm{NH}_{2}$ & $\mathrm{NH}_{2}{ }^{+}$ & $\mathrm{N}_{2} \mathrm{H}$ & $\mathrm{H}_{2} \mathrm{O}$ & $\mathrm{H}_{2} \mathrm{O}^{+}$ \\
$\mathrm{C}_{2} \mathrm{H}$ & $\mathrm{C}_{2} \mathrm{H}^{+}$ & $\mathrm{HCN}$ & $\mathrm{HCN}^{+}$ & $\mathrm{HNC}$ & $\mathrm{HCO}$ & $\mathrm{HCO}^{+}$ & $\mathrm{N}_{2} \mathrm{H}^{+}$ \\
$\mathrm{HNO}$ & $\mathrm{HNO}^{+}$ & $\mathrm{HO}_{2}{ }^{+}$ & $\mathrm{NCO}$ & $\mathrm{C}_{3}$ & $\mathrm{C}_{3}{ }^{+}$ & $\mathrm{C}_{2} \mathrm{~N}^{+}$ & $\mathrm{CO}_{2}$
\end{tabular}

$\mathrm{CO}_{2}{ }^{+}$

Tetratomics

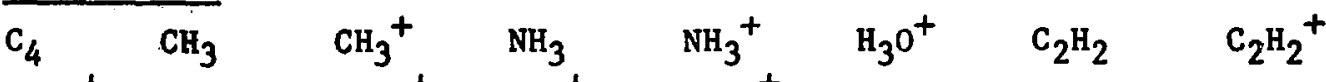
$\mathrm{H}_{2} \mathrm{CN}^{+} \quad \mathrm{H}_{2} \mathrm{CO} \quad \mathrm{H}_{2} \mathrm{CO}^{+} \quad \mathrm{C}_{3} \mathrm{H}^{+} \quad \mathrm{CO}_{2} \mathrm{H}^{+}$

Pentatomics

\begin{tabular}{|c|c|c|c|c|c|c|}
\hline & $\mathrm{CH}_{4}$ & $\mathrm{CH}_{4}^{+}$ & $\mathrm{C}_{4} \mathrm{H}$ & $\mathrm{N}_{2} \mathrm{H}_{2}$ & $\mathrm{NH}_{4}^{+}$ & $\mathrm{CH}_{3} \mathrm{O}^{+}$ \\
\hline $\mathrm{C}_{2} \mathrm{H}_{3}{ }^{+}$ & $\mathrm{C}_{3} \mathrm{H}_{2}{ }^{+}$ & $\mathrm{CH}_{2} \mathrm{OH}^{+}$ & $\mathrm{H}_{2} \mathrm{CO}_{2}$ & $\mathrm{H}_{2} \mathrm{CO}_{2}^{+}$ & $\mathrm{CH}_{2} \mathrm{CN}^{+}$ & $\mathrm{CH}_{3} \mathrm{~N}$ \\
\hline
\end{tabular}

Hexatomics
$\mathrm{CH}_{5}+\mathrm{C}_{2} \mathrm{H}_{4}$
$\mathrm{C}_{2} \mathrm{H}_{4}{ }^{+} \quad \mathrm{CH}_{4} \mathrm{~N}^{+}\left(=\mathrm{CH}_{2} \mathrm{NH}_{2}^{+}\right)$
$\mathrm{C}_{3} \mathrm{H}_{3}{ }^{+}$
$\mathrm{CH}_{3} \mathrm{OH}$
$\mathrm{CH}_{3} \mathrm{OH}^{+}$
$\mathrm{CH}_{3} \mathrm{CN} \quad \mathrm{CH}_{3} \mathrm{CO}^{+} \quad \mathrm{HC}_{4} \mathrm{H}$
$\mathrm{C}_{4} \mathrm{H}_{2}+\mathrm{N}_{2} \mathrm{H}_{3}$
$\mathrm{C}_{3} \mathrm{H}_{2} \mathrm{~N}^{+}\left(=\mathrm{C}_{2} \mathrm{H}_{2} \mathrm{CN}^{+}\right.$?)
$\mathrm{H}_{2} \mathrm{~N}_{2} \mathrm{H}_{2}$

\section{Suprahexatomics}

$\begin{array}{llllllll}\mathrm{CH}_{3} \mathrm{NH}_{2} & \mathrm{C}_{2} \mathrm{H}_{5} & \mathrm{C}_{2} \mathrm{H}_{5}^{+} & \mathrm{H}_{2} \mathrm{C}_{3} \mathrm{H}_{2} & \mathrm{C}_{3} \mathrm{H}_{4}^{+} & \mathrm{C}_{4} \mathrm{H}_{3}^{+} & \mathrm{C}_{3} \mathrm{H}_{3} \mathrm{O}^{+} & \mathrm{H}_{3} \mathrm{O}^{+} \cdot \mathrm{H}_{2} \mathrm{O}\left(-\mathrm{H}_{3} \mathrm{O}^{+} \cdot \mathrm{W}\right) \\ \mathrm{C}_{2} \mathrm{H}_{6} & \mathrm{C}_{2} \mathrm{H}_{6}{ }^{+} & \mathrm{C}_{3} \mathrm{H}_{5}{ }^{+} & \mathrm{C}_{2} \mathrm{H}_{7}^{+} & \mathrm{C}_{3} \mathrm{H}_{6}{ }^{+} & \mathrm{C}_{4} \mathrm{H}_{5}^{+} & \mathrm{C}_{3} \mathrm{H}_{7}^{+} & \mathrm{C}_{2} \mathrm{H}_{9}^{+} \\ \mathrm{C}_{3} \mathrm{H}_{8}^{+} & \mathrm{C}_{4} \mathrm{H}_{7}^{+} & \mathrm{C}_{3} \mathrm{H}_{9}^{+} & \mathrm{C}_{3} \mathrm{H}_{10}{ }^{+} & \mathrm{C}_{4} \mathrm{H}_{9}^{+} & \mathrm{C}_{3} \mathrm{H}_{11}^{+} & & \end{array}$




\section{Reactions}

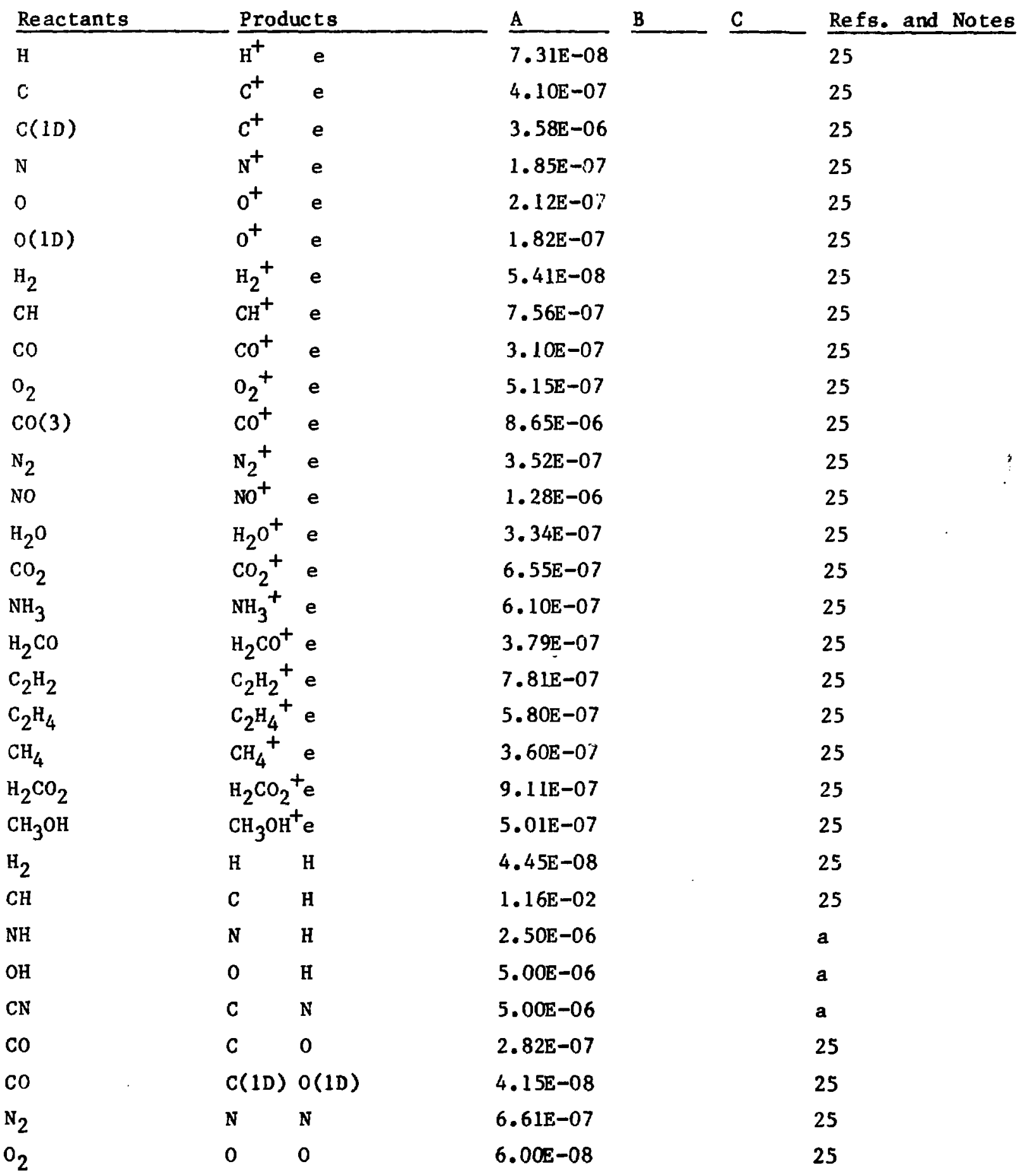


Table IV (cont)

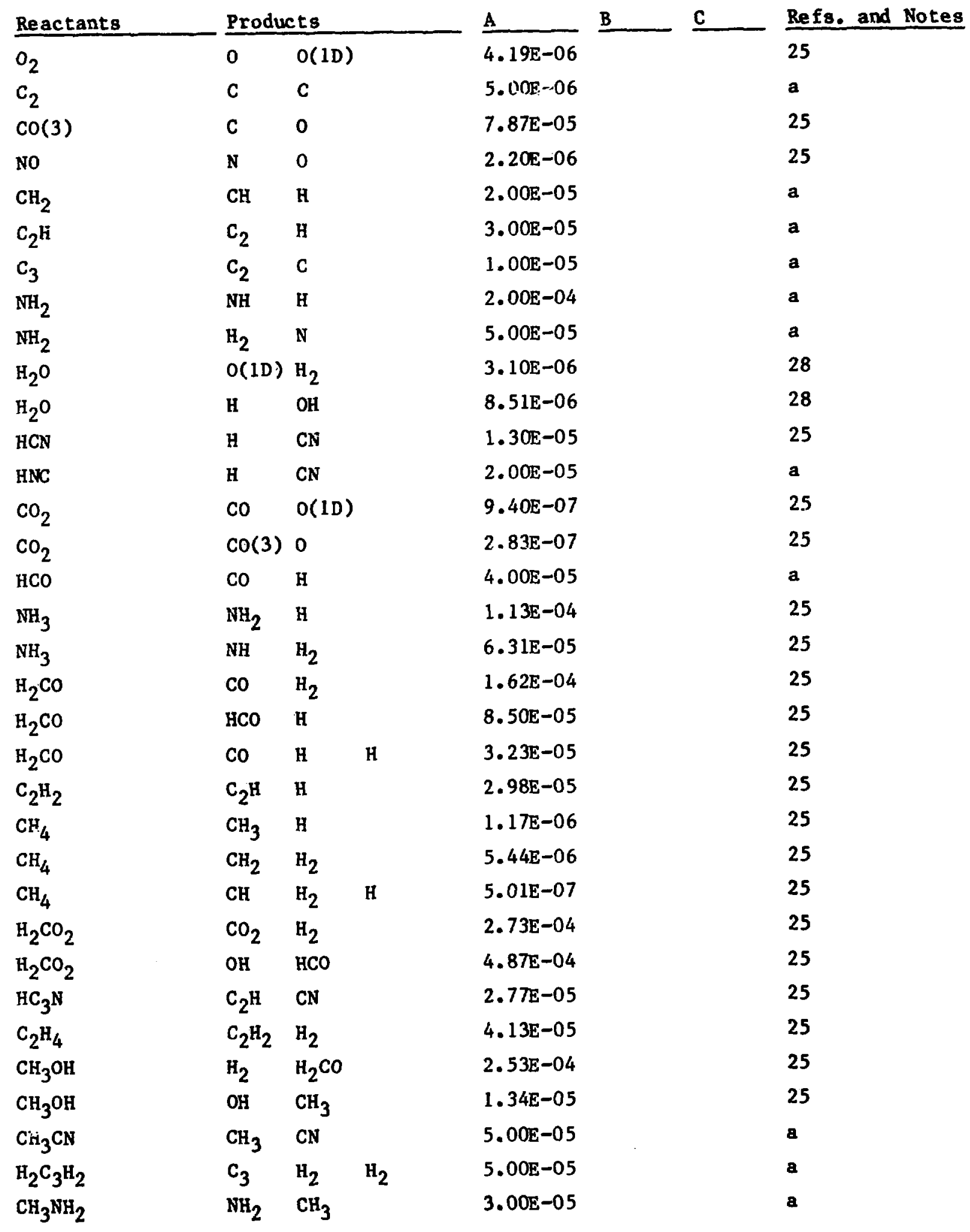


Table IV (cont)

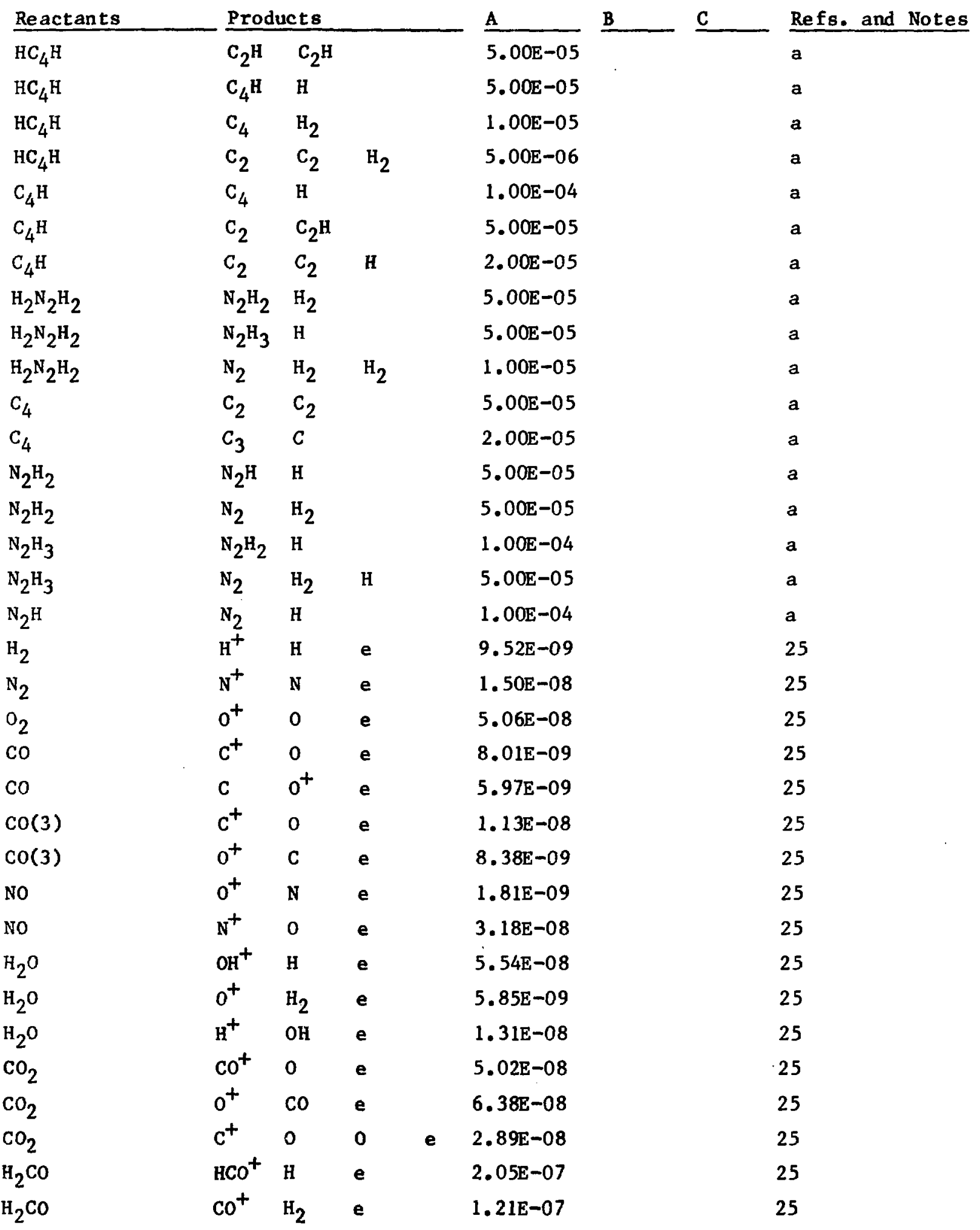


Table IV (cont)

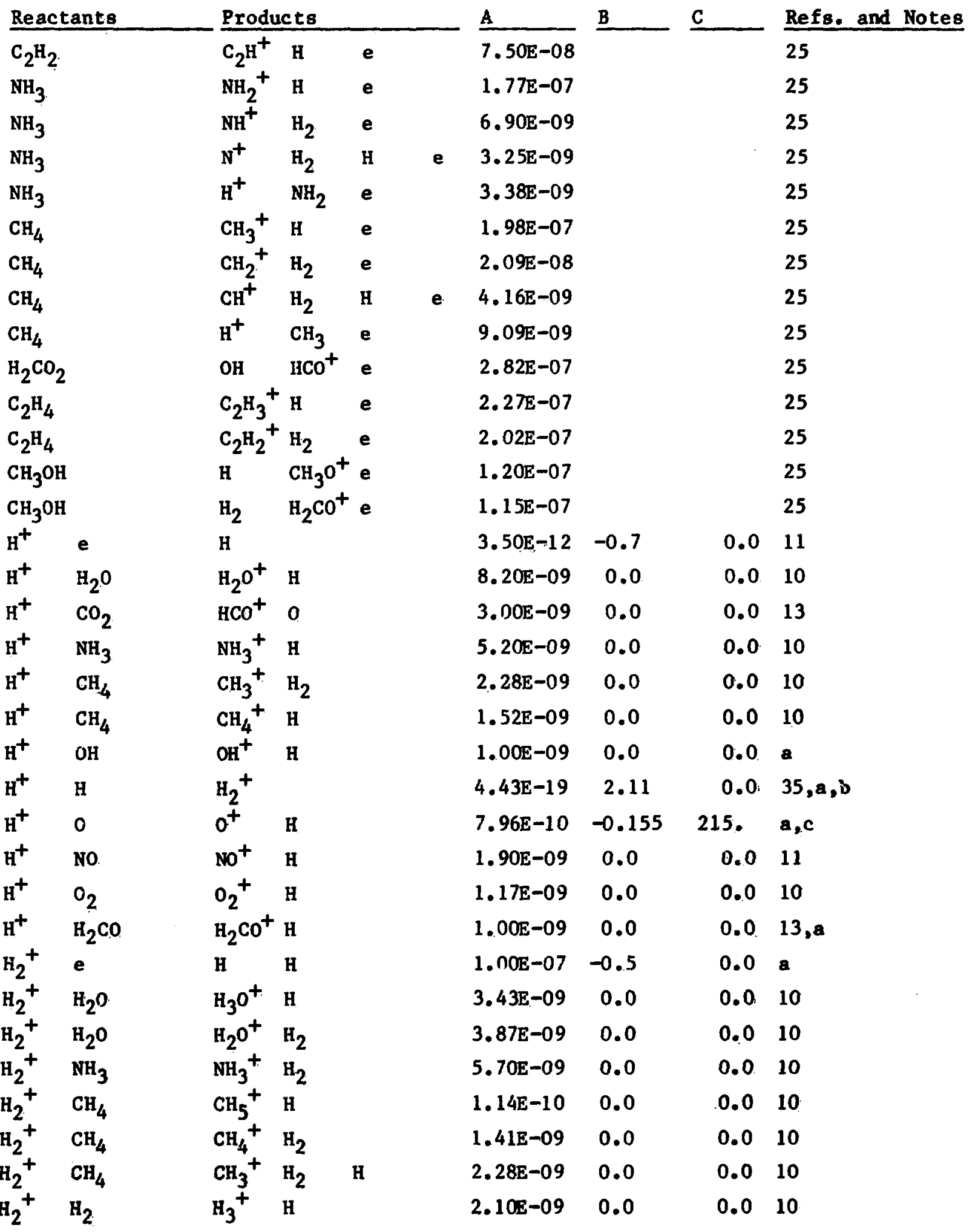


Table IV (cont)

\begin{tabular}{|c|c|c|c|c|c|c|c|}
\hline Reactants & Products & & & $\underline{A}$ & $\underline{\mathbf{B}}$ & $\underline{\mathbf{C}}$ & Refs. and \\
\hline $\mathrm{H}_{2}^{+} \quad \mathrm{C}$ & $\mathrm{CH}^{+} \quad \mathrm{H}$ & & & $1.00 \mathrm{~s}-09$ & 0.0 & 0.0 & $\mathbf{a}$ \\
\hline $\mathrm{H}_{2}{ }^{+} \quad \mathrm{N}$ & $\mathrm{NH}^{+}$ & & & $1.00 E-09$ & 0.0 & 0.0 & a \\
\hline $\mathrm{H}_{2}{ }^{+}$ & $\mathrm{OH}^{+}$ & & & $1.00 \mathrm{E}-09$ & 0.0 & 0.0 & $\mathbf{a}$ \\
\hline $\mathrm{H}_{2}^{+}$ & $\mathrm{CO}^{+}$ & & & $6.44 E-10$ & 0.0 & 0.0 & 10 \\
\hline $\mathrm{H}_{2}^{+}$ & $\mathrm{HCO}^{+}$ & & & $2.16 E-09$ & 0.0 & 0.0 & 10 \\
\hline $\mathrm{H}_{2}^{+}$ & $\mathrm{HO}_{2}{ }^{+}$ & & & $1.92 \mathrm{E}-09$ & 0.0 & 0.0 & 10 \\
\hline $\mathrm{H}_{2}^{+}$ & $\mathrm{N}_{2} \mathrm{H}^{+} \quad \mathrm{H}$ & & & $2.00 \mathrm{E}-09$ & 0.0 & 0.0 & 10 \\
\hline $\mathrm{H}_{2}^{+}$ & $\mathrm{O}_{2}^{+} \quad \mathrm{H}_{2}$ & & & $7.83 \mathrm{E}-10$ & 0.0 & 0.0 & 10 \\
\hline $\mathrm{C}_{2} \mathrm{H}_{2}$ & $\mathrm{C}_{2} \mathrm{H}_{3}^{+} \mathrm{H}$ & & & $4.77 \mathrm{E}-10$ & 0.0 & 0.0 & 10 \\
\hline $\mathrm{H}_{2}{ }^{+} \quad \mathrm{C}_{2} \mathrm{H}_{2}$ & $\mathrm{C}_{2} \mathrm{H}_{2}{ }^{+} \mathrm{H}_{2}$ & & & $4.82 \mathrm{E}-09$ & 0.0 & 0.0 & 10 \\
\hline $\mathrm{C}_{2} \mathrm{H}_{4}$ & $\mathrm{C}_{2} \mathrm{H}_{4}{ }^{+} \mathrm{H}_{2}$ & & & $2.20 \mathrm{E}-09$ & 0.0 & 0.0 & 10 \\
\hline $\mathrm{C}_{2} \mathrm{H}_{4}$ & $\mathrm{C}_{2} \mathrm{H}_{3}{ }^{+} \mathrm{H}_{2}$ & $\mathrm{H}$ & & $1.81 E-09$ & 0.0 & 0.0 & 10 \\
\hline $\mathrm{C}_{2} \mathrm{H}_{4}$ & $\mathrm{C}_{2} \mathrm{H}_{2}^{+} \mathrm{H}_{2}$ & $\mathrm{H}_{2}$ & & $8.80 E-10$ & 0.0 & 0.0 & 10 \\
\hline $\mathrm{C}_{2} \mathrm{H}_{6}$ & $\mathrm{C}_{2} \mathrm{H}_{6}^{+}{ }^{+} \mathrm{H}_{2}$ & & & $2.94 \mathrm{E}-10$ & 0.0 & 0.0 & 10 \\
\hline $\mathrm{C}_{2} \dot{\mathrm{H}}_{6}$ & $\mathrm{C}_{2} \mathrm{H}_{5}^{+} \mathrm{H}_{2}$ & $\mathrm{H}$ & & $1.37 E-09$ & 0.0 & 0.0 & 10 \\
\hline $\mathrm{c}_{2} \mathrm{H}_{6}$ & $\mathrm{C}_{2} \mathrm{H}_{4}^{+} \mathrm{H}_{2}$ & $\mathrm{H}_{2}$ & & $2.35 E-09$ & 0.0 & 0.0 & 10 \\
\hline $\mathrm{C}_{2} \mathrm{H}_{6}$ & $\mathrm{C}_{2} \mathrm{H}_{3}{ }^{+} \mathrm{H}_{2}$ & $\mathrm{H}_{2}$ & $H$ & $6.86 \mathrm{E}-10$ & 0.0 & 0.0 & 10 \\
\hline $\mathrm{C}_{2} \mathrm{H}_{6}$ & $\mathrm{C}_{2} \mathrm{H}_{2}^{+} \mathrm{H}_{2}$ & $\mathrm{H}_{2}$ & $\mathrm{H}_{2}$ & $1.96 \mathrm{E}-10$ & 0.0 & 0.0 & 10 \\
\hline $\mathrm{H}_{3}{ }^{+}$ & $\mathrm{H}_{2} \quad \mathrm{H}$ & & & $2.10 E-07$ & -0.5 & 0.0 & 52 \\
\hline $\mathrm{H}_{3}{ }^{+}$ & H & $\mathbf{H}$ & & $2.10 E-07$ & -0.5 & 0.0 & 52 \\
\hline $\mathrm{H}_{2} \mathrm{O}$ & $\mathrm{H}_{3} \mathrm{O}^{+} \quad \mathrm{H}_{2}$ & & & $5.90 \mathrm{E}-09$ & 0.0 & 0.0 & 10 \\
\hline $\mathrm{CO}_{2}$ & $\mathrm{CO}_{2} \mathrm{H}^{+} \mathrm{H}_{2}$ & & & $1.90 E-09$ & 0.0 & 0.0 & 13 \\
\hline $\mathrm{NH}_{3}$ & $\mathrm{NH}_{4}^{+} \mathrm{H}_{2}$ & & & $4.70 \mathrm{E}-09$ & 0.0 & 0.0 & 10 \\
\hline $\mathrm{CH}_{4}$ & $\mathrm{CH}_{5}{ }^{+}$ & & & $2.40 \mathrm{E}-09$ & 0.0 & 0.0 & 10 \\
\hline $\mathrm{H}_{3}{ }^{+}$ & $\mathrm{CH}^{+}$ & & & $2.00 E-09$ & 0.0 & 0.0 & $13, a$ \\
\hline $\mathrm{H}_{3}{ }^{+}$ & $\mathrm{OH}^{+}$ & & & $8.00 E-10$ & 0.0 & 0.0 & $16, a$ \\
\hline $\mathrm{H}_{3}{ }^{+}$ & $\mathrm{H}_{2} \mathrm{O}^{+}$ & & & $2.00 \mathrm{E}-09$ & 0.0 & 0.0 & $13, a$ \\
\hline $\mathrm{H}_{3}{ }^{+}$ & $\mathrm{HCO}^{+}$ & & & $1.70 E-09$ & 0.0 & 0.0 & 10 \\
\hline $\mathrm{H}_{3}{ }^{+}$ & $\mathrm{HO}_{2}{ }^{+} \mathrm{H}_{2}$ & & & $6.40 \mathrm{E}-10$ & 0.0 & 0.0 & 10 \\
\hline $\mathrm{H}_{2} \mathrm{CO}$ & $\mathrm{CH}_{2} \mathrm{OH}^{+} \mathrm{H}_{2}$ & & & $2.00 E-09$ & 0.0 & 0.0 & $13, a$ \\
\hline $\mathrm{C}_{2} \mathrm{H}_{2}$ & $\mathrm{C}_{2} \mathrm{H}_{3}{ }^{+} \mathrm{H}_{2}$ & & & $3.50 \mathrm{E}-09$ & 0.0 & 0.0 & 10 \\
\hline $\mathrm{C}_{2} \mathrm{H}_{4}$ & $\mathrm{C}_{2} \mathrm{H}_{5}{ }^{+} \mathrm{H}_{2}$ & & & $1.44 \mathrm{E}-09$ & 0.0 & 0.0 & 10 \\
\hline $\mathrm{C}_{2} \mathrm{H}_{4}$ & $\mathrm{C}_{2} \mathrm{H}_{3}{ }^{+} \mathrm{H}_{2}$ & $\mathrm{H}_{2}$ & & $2.16 \mathrm{E}-09$ & 0.0 & 0.0 & 10 \\
\hline $\mathrm{C}_{2} \mathrm{H}_{6}$ & $\mathrm{C}_{2} \mathrm{H}_{5}{ }^{+} \mathrm{H}_{2}$ & $\mathrm{H}_{2}$ & & $3.40 \mathrm{E}-09$ & 0.0 & 0.0 & 10 \\
\hline
\end{tabular}


Table IV (cont)

\begin{tabular}{|c|c|c|c|c|c|c|c|c|}
\hline Reactants & & Produc & cts & & $\underline{A}$ & $\underline{\mathbf{B}}$ & C & Refo. and \\
\hline $\mathrm{H}_{3}^{+} \quad \mathrm{HCN}$ & & $\mathrm{H}_{2} \mathrm{CN}^{+}$ & $\mathrm{H}_{2}$ & & $8.00 \mathrm{E}-09$ & 0.0 & 0.0 & 10 \\
\hline $\mathrm{H}_{3}^{+}$ & & $\mathrm{H}_{2} \mathrm{CO}^{+}$ & $\mathrm{H}_{2}$ & & $1.00 \mathrm{E}-09$ & 0.0 & 0.0 & $\mathbf{a}$ \\
\hline $\mathrm{H}_{3}^{+}$NO & & $\mathrm{HNO}^{+}$ & $\mathrm{H}_{2}$ & & $1.40 E-09$ & 0.0 & 0.0 & 12 \\
\hline $\mathrm{H}_{3}^{+} \quad \mathrm{N}_{2}$ & - & $\mathrm{N}_{2} \mathrm{H}^{+}$ & $\mathrm{H}_{2}$ & & $1.70 \mathrm{E}-09$ & 0.0 & 0.0 & 10 \\
\hline $\mathrm{H}_{3}^{+} \quad \mathrm{CN}$ & & $\mathrm{HCN}^{+}$ & $\mathrm{H}_{2}$ & & $2.00 \mathrm{E}-09$ & 0.0 & 0.0 & $13, a$ \\
\hline $\mathrm{H}_{3}^{+} \quad \mathrm{I}_{2}$ & $\mathbf{M}$ & $\mathrm{H}_{5}{ }^{+}$ & $\mathbf{M}$ & & $1.00 E-30$ & 0.0 & 0.0 & $17, \mathrm{a}$ \\
\hline $\mathrm{H}_{5}^{+}$ & & $\mathrm{H}_{2}$ & $\mathrm{H}_{2}$ & H & $3.00 \pm-06$ & -0.5 & 0.0 & $18, a$ \\
\hline $\mathrm{H}_{5}^{+} \quad \mathrm{CH}_{4}$ & & $\mathrm{CH}_{5}^{+}$ & $\mathrm{H}_{2}$ & $\mathrm{H}_{2}$ & $2.00 \mathrm{E}-09$ & 0.0 & 0.0 & $17, a$ \\
\hline$c^{+}$ & & C & & & $3.50 \mathrm{E}-12$ & -0.7 & 0.0 & $\mathbf{a}$ \\
\hline $\mathrm{H}_{2} \mathrm{O}$ & & $\mathrm{HCO}^{+}$ & $\mathrm{H}$ & & $2.70 E-09$ & 0.0 & 0.0 & 10 \\
\hline $\mathrm{CO}_{2}$ & & $\mathrm{Co}^{+}$ & $\mathrm{CO}$ & & $1.10 E-09$ & 0.0 & 0.0 & 10 \\
\hline $\mathrm{NH}_{3}$ & & $\mathrm{NH}_{3}{ }^{+}$ & C & & $1.15 E-09$ & 0.0 & 0.0 & 10 \\
\hline $\mathrm{NH}_{3}$ & & $\mathrm{HCN}^{+}$ & $\mathrm{H}_{2}$ & & $7.00 E-11$ & 0.0 & 0.0 & 10 \\
\hline $\mathrm{NH}_{3}$ & & $\mathrm{H}_{2} \mathrm{CN}^{+}$ & $\mathrm{H}$ & & $1.08 E-09$ & 0.0 & 0.0 & 10 \\
\hline $\mathrm{CH}_{4}$ & & $\mathrm{C}_{2} \mathrm{H}_{3}{ }^{+}$ & $\mathbf{H}$ & & $1.03 E-00$ & 0.0 & 0.0 & 10 \\
\hline $\mathrm{CH}_{4}$ & & $\mathrm{C}_{2} \mathrm{H}_{2}{ }^{+}$ & $\mathrm{H}_{2}$ & & $4,20 \mathrm{E}-10$ & 0.0 & 0.0 & 10 \\
\hline NH & & $\mathrm{H}^{+}$ & $\mathrm{CN}$ & & $1.00 \mathrm{E}-09$ & 0.0 & 0.0 & a \\
\hline NH & & $\mathrm{CN}^{+}$ & $\mathbf{H}$ & & $1.00 \mathrm{E}-09$ & 0.0 & 0.0 & $\mathbf{a}$ \\
\hline $\mathrm{NH}_{2}$ & & $\mathrm{H}^{+}$ & $\mathrm{HCN}$ & & $1.00 \mathrm{E}-09$ & 0.0 & 0.0 & $\mathbf{a}$ \\
\hline $\mathrm{NH}_{2}$ & & $\mathrm{HCN}^{+}$ & $\mathbf{H}$ & & $1.00 \mathrm{E}-09$ & 0.0 & 0.0 & $\mathbf{a}$ \\
\hline $\mathrm{H}_{2}$ & & $\mathrm{CH}_{2}{ }^{+}$ & & & $3.00 E-15$ & -1.0 & 0.0 & $19, a$ \\
\hline $\mathrm{OH}$ & & $\mathrm{CO}^{+}$ & $\mathbf{H}$ & & $1.00 E-09$ & 0.0 & 0.0 & $\mathbf{a}$ \\
\hline $\mathrm{OH}$ & & $\mathrm{H}^{+}$ & Co & & $1.00 E-09$ & 0.0 & 0.0 & $\mathbf{a}$ \\
\hline$c^{+}$ & & $\hat{v}_{2}{ }^{+}$ & $\mathbf{H}$ & & $2.00 E-09$ & 0.0 & 0.0 & $13, a$ \\
\hline $\mathrm{CH}_{2}$ & & $\mathrm{C}_{2} \mathrm{H}^{+}$ & H & & $2.00 z-09$ & 0.0 & 0.0 & $13, a$ \\
\hline $\mathrm{CH}_{3}$ & & $\mathrm{C}_{2} \mathrm{H}_{2}^{+}$ & $\mathbf{H}$ & & $1.00 \mathrm{E}-09$ & 0.0 & 0.0 & $\mathbf{a}$ \\
\hline $\mathrm{C}_{2} \mathrm{H}$ & & $\mathrm{c}_{3}{ }^{+}$ & $\mathbf{H}$ & & $1.00 \mathrm{E}-09$ & 0.0 & 0.0 & $\mathbf{a}$ \\
\hline $\mathrm{H}_{2} \mathrm{CO}$ & & $\mathrm{CH}_{2}^{+}$ & $\mathrm{CO}$ & & $2.11 E-09$ & 0.0 & 0.0 & 10 \\
\hline $\mathrm{n}_{2} \mathrm{CO}$ & & $\mathrm{HCO}^{+}$ & $\mathrm{CH}$ & & $9.20 \mathrm{E}-10$ & 0.0 & 0.0 & 10 \\
\hline $\mathrm{H}_{2} \mathrm{CO}$ & & $\mathrm{H}_{2} \mathrm{CO}^{+}$ & C & & $1.36 \mathrm{E}-09$ & 0.0 & 0.0 & 10 \\
\hline $\mathrm{o}_{2}$ & & $\mathrm{Co}^{+}$ & 0 & & $7.80 \mathrm{E}-10$ & 0.0 & 0.0 & 10 \\
\hline $\mathrm{O}_{2}$ & & $0^{+}$ & co & & $4.40 \mathrm{E}-10$ & 0.0 & 0.0 & 10 \\
\hline No & & $\mathrm{NO}^{+}$ & $\mathrm{C}$ & & $8.50 \mathrm{E}-10$ & 0.0 & 0.0 & 10 \\
\hline $\mathrm{HCN}$ & & $\mathrm{C}_{2} \mathrm{~N}^{+}$ & $\mathbf{H}$ & & $3.20 \mathrm{E}-09$ & 0.0 & 0.0 & 10 \\
\hline
\end{tabular}


Table IV (cont)

Reactants

$\mathrm{C}^{+} \mathrm{H}$

$\mathrm{CH}^{+} \mathrm{e}$

$\mathrm{CH}^{+} \mathrm{e}$

$\mathrm{CH}^{+} \quad \mathrm{H}_{2} \mathrm{O}$

$\mathrm{CH}^{+} \quad \mathrm{H}_{2} \mathrm{O}$

$\mathrm{CH}^{+} \quad \mathrm{H}_{2} \mathrm{O}$

$\mathrm{CH}^{+} \quad \mathrm{CO}_{2}$

$\mathrm{CH}^{+} \mathrm{NH}_{3}$

$\mathrm{CH}^{+} \mathrm{NH}_{3}$

$\mathrm{CH}^{+} \quad \mathrm{NH}_{3}$

$\mathrm{CH}^{+} \mathrm{CH}_{4}$

$\mathrm{CH}^{+} \mathrm{CH}_{4}$

$\mathrm{CH}^{+} \mathrm{CH}_{4}$

$\mathrm{CH}^{+} \quad \mathrm{H}_{2} \mathrm{CO}$

$\mathrm{CH}^{+} \mathrm{HCN}$

$\mathrm{CH}^{+} \quad \mathrm{C}_{2} \mathrm{H}_{2}$

$\mathrm{CH}^{+} \quad \mathrm{C}_{2} \mathrm{H}_{4}$

$\mathrm{CH}^{+} \mathrm{HCO}$

$\mathrm{CH}^{+} \quad \mathrm{NH}_{2}$

$\mathrm{CH}^{+} \quad \mathrm{H}_{2}$

$\mathrm{CH}^{+} \mathrm{N}$

$\mathrm{CH}^{+} \mathrm{O}$

$\mathrm{CH}^{+} \quad \mathrm{O}_{2}$

$\mathrm{CH}^{+} \mathrm{O}_{2}$

$\mathrm{CH}^{+} \mathrm{O}_{2}$

$\mathrm{CH}_{2}{ }^{+} \mathrm{e}$

$\mathrm{CH}_{2}{ }^{+} \mathrm{H}_{2} \mathrm{O}^{\circ}$

$\mathrm{CH}_{2}{ }^{+} \mathrm{CO}_{2}$

$\mathrm{CH}_{2}{ }^{+} \mathrm{NH}_{3}$

$\mathrm{CH}_{2}{ }^{+} \mathrm{NH}_{3}$

$\mathrm{CH}_{2}{ }^{+} \mathrm{CH}_{4}$

$\mathrm{CH}_{2}+\mathrm{CH}_{4}$

$\mathrm{CH}_{2}{ }^{+} \mathrm{CH}_{4}$

$\mathrm{CH}_{2}{ }^{+} \mathrm{CH}_{4}$
Products

$\mathrm{CH}^{+}$

C $\mathrm{H}$

$\mathrm{CH}$

$\mathrm{HCO}^{+} \mathrm{H}_{2}$

$\mathrm{H}_{2} \mathrm{CO}^{+} \mathrm{H}$

$\mathrm{H}_{3} \mathrm{O}^{+} \mathrm{C}$

$\mathrm{HCO}^{+} \mathrm{CO}$

$\mathrm{H}_{2} \mathrm{CN}^{+} \mathrm{H}_{2}$

$\mathrm{NH}_{3}{ }^{+} \mathrm{CH}$

$\mathrm{NH}_{4}{ }^{+} \mathrm{C}$

$\mathrm{C}_{2} \mathrm{H}_{4}+\mathrm{H}$

$\mathrm{C}_{2} \mathrm{H}_{3}{ }^{+} \mathrm{H}_{2}$

$\mathrm{C}_{2} \mathrm{H}_{2}{ }^{+} \mathrm{H}_{2} \quad \mathrm{H}$

$\mathrm{CH}_{2} \mathrm{OH}^{+} \mathrm{C}$

$\mathrm{H}_{2} \mathrm{CN}^{+} \mathrm{C}$

$\mathrm{C}_{2} \mathrm{H}_{3}+\mathrm{C}$

$\mathrm{C}_{2} \mathrm{H}_{5}{ }^{+} \mathrm{C}$

$\mathrm{H}_{2} \mathrm{CO}^{+} \mathrm{C}$

$\mathrm{NH}_{3}{ }^{+} \mathrm{C}$

$\mathrm{CH}_{2}{ }^{+} \mathrm{H}$

$\mathrm{CN}^{+} \mathrm{H}$

$\mathrm{CO}^{+} \mathrm{H}$

$\mathrm{HCO}^{+} \mathrm{O}$

$\mathrm{O}^{+} \mathrm{HCO}$

$\mathrm{CO}^{+} \mathrm{OH}$

$\mathrm{CH} \quad \mathrm{H}$

$\mathrm{CH}_{2} \mathrm{OH}^{+} \mathrm{H}$

$\mathrm{H}_{2} \mathrm{CO}^{+} \mathrm{CO}$

$\mathrm{NH}_{4}^{+} \mathrm{CH}$

$\mathrm{CH}_{4} \mathrm{~N}^{+} \mathrm{H}$

$\mathrm{C}_{2} \mathrm{H}_{5}+\mathrm{H}$

$\mathrm{C}_{2} \mathrm{H}_{4}+\mathrm{H}_{2}$

$\mathrm{C}_{2} \mathrm{H}_{3}+\mathrm{H}_{2} \quad \mathrm{H}$

$\mathrm{C}_{2} \mathrm{H}_{2}{ }^{+} \mathrm{H}_{2}$
$\frac{A}{5.67 \mathrm{E}-18}$

$\frac{B}{-1.0}$

C

1. OOE $-07-0.5$

$1.05 \mathrm{E}-10-0.7$

2. 32E-09 0.0

2. $90 \mathrm{E}-10 \quad 0.0$

2. $90 \mathrm{E}-10 \quad 0.0$

$1.60 \mathrm{E}-09 \quad 0.0$

$1.84 E-09 \quad 0.0$

4. $60 \mathrm{E}-10 \quad 0.0$

4. $00 \mathrm{E}-10 \quad 0.0$

$7.70 \mathrm{E}-11 \quad 0.0$

$1.06 \mathrm{E}-09 \quad 0.0$

$1.55 E-10 \quad 0.0$

$1.00 \mathrm{E}-09 \quad 0.0$

1.00E-09 0.0

1. $00 \mathrm{E}-09 \quad 0.0$

1. $00 \mathrm{E}-09 \quad 0.0$

$1.00 \mathrm{E}-090.0$

$1.00 \mathrm{E}-09 \quad 0.0$

1.01E-09 0.0

$1.00 \mathrm{E}-09 \quad 0.0$

$1.00 \mathrm{E}-09 \quad 0.0$

9.22E-10 0.0

$2.40 \mathrm{E}-110.0$

$2.40 \mathrm{E}-11 \quad 0.0$

5.10E-07 -0.5

1. 20E-09 0.0

1. 60E-09 0.0

$5.25 \mathrm{E}-10 \quad 0.0$

$1.98 \mathrm{E}-09 \quad 0.0$

$2.88 \mathrm{E}-10 \quad 0.0$

5.04E-10 0.0

2.64E-10 0.0

$1.44 \mathrm{E}-10 \quad 0.0$
Refs. and Notes

$0.0 \quad 33, a$

$0.020,34, a$

$0.020,33, a, d$

$0.038, e$

$0.038, e$

$0.038, e$

0.038

0.038

0.038

0.038

0.010

0.010

0.010

$0.0 \mathrm{a}$

0.0 a

$0.0 a$

0.0 a

0.0 a

0.0 a

$0.0 \quad 10$

$0.014, a$

0.0 a

$0.0 \quad 42$,e

0.042 ,e

0.042 ,e

0.052

0.010

0.038

0.010

0.010

0.010

0.010

0.010

0.010 
Table IV (cont)

Reactants

$\mathrm{CH}_{2}^{+} \mathrm{H}_{2}$

$\mathrm{CH}_{2}+\mathrm{O}$

$\mathrm{CH}_{2}+\mathrm{O}_{2}$

$\mathrm{CH}_{2}^{+} \mathrm{O}_{2}$

$\mathrm{CH}_{3}^{+} \mathrm{e}$

$\mathrm{CH}_{3}^{+} \mathrm{e}$

$\mathrm{CH}_{3}^{+} \mathrm{e}$

$\mathrm{CH}_{3}{ }^{+} \mathrm{NH}_{3}$

$\mathrm{CH}_{3}{ }^{+} \mathrm{NH}_{3}$

$\mathrm{CH}_{3}{ }^{+} \mathrm{CH}_{4}$

$\mathrm{CH}_{3}^{+} \mathrm{C}$

$\mathrm{CH}_{3}^{+} \mathrm{N}$

$\mathrm{CH}_{3}{ }^{+} \mathrm{N}$

$\mathrm{CH}_{3}{ }^{+} \mathrm{O}$

$\mathrm{CH}_{3}{ }^{+} \mathrm{O}_{2}$

$\mathrm{CH}_{3}^{+}$NO

$\mathrm{CH}_{3}{ }^{+} \mathrm{C}_{2} \mathrm{H}_{2}$

$\mathrm{CH}_{3}{ }^{+} \mathrm{C}_{2} \mathrm{H}_{4}$

$\mathrm{CH}_{3}+\mathrm{C}_{2} \mathrm{H}_{4}$

$\mathrm{CH}_{3}{ }^{+} \mathrm{C}_{2} \mathrm{H}_{4}$

$\mathrm{CH}_{3}{ }^{+} \mathrm{C}_{2} \mathrm{H}_{6}$

$\mathrm{CH}_{3}{ }^{+} \mathrm{C}_{2} \mathrm{H}_{6}$

$\mathrm{CH}_{3}{ }^{+} \mathrm{C}_{2} \mathrm{H}_{6}$

$\mathrm{CH}_{3}{ }^{+} \mathrm{H}_{2} \mathrm{CO}$

$\mathrm{CH}_{3}{ }^{+} \mathrm{H}_{2} \quad \mathrm{M}$

$\mathrm{CH}_{4}^{+} \mathrm{e}$

$\mathrm{CH}_{4}^{+} \mathrm{e}$

$\mathrm{CH}_{4}^{+} \mathrm{e}$

$\mathrm{CH}_{4}^{+} \mathrm{e}$

$\mathrm{CH}_{4}^{+} \mathrm{e}$

$\mathrm{CH}_{4}{ }^{+} \mathrm{H}_{2} \mathrm{O}$

$\mathrm{CH}_{4}{ }^{+} \mathrm{CO}_{2}$

$\mathrm{CH}_{4}{ }^{+} \mathrm{NH}_{3}$

$\mathrm{CH}_{4}{ }^{+} \mathrm{NH}_{3}$
Products

$\mathrm{CH}_{3}^{+} \mathrm{H}$

$\mathrm{HCO}^{+} \mathrm{H}$

$\mathrm{HCO}^{+} \mathrm{OH}$

$\mathrm{H}_{2} \mathrm{CO}^{+} \mathrm{O}$

$\mathrm{CH}_{2} \quad \mathrm{H}$

CH $\mathrm{H}_{2}$

$\mathrm{CH} \quad \mathrm{H}$

$\mathrm{NH}_{4}{ }^{+} \mathrm{CH}_{2}$

$\mathrm{CH}_{4} \mathrm{~N}^{+} \mathrm{H}_{2}$

$\mathrm{C}_{2} \mathrm{H}_{5}{ }^{+} \mathrm{H}_{2}$

$\mathrm{C}_{2} \mathrm{H}^{+} \mathrm{H}_{2}$

$\mathrm{H}_{2} \mathrm{CN}^{+} \mathrm{H}$

$\mathrm{HCN}^{+} \mathrm{H}_{2}$

$\mathrm{HCO}^{+} \mathrm{H}_{2}$

$\mathrm{HCO}^{+} \mathrm{H}_{2} \mathrm{O}$

$\mathrm{NO}^{+} \mathrm{CH}_{3}$

$\mathrm{C}_{3} \mathrm{H}_{3}{ }^{+} \mathrm{H}_{2}$

$\mathrm{C}_{2} \mathrm{H}_{3}+\mathrm{CH}_{4}$

$\mathrm{C}_{3} \mathrm{H}_{3}{ }^{+} \mathrm{H}_{2} \quad \mathrm{H}_{2}$

$\mathrm{C}_{3} \mathrm{H}_{5}+\mathrm{H}_{2}$

$\mathrm{C}_{2} \mathrm{H}_{5}+\mathrm{CH}_{4}$

$\mathrm{C}_{3} \mathrm{H}_{5}{ }^{+} \mathrm{H}_{2}$

$\mathrm{C}_{3} \mathrm{H}_{7}+\mathrm{H}_{2}$

$\mathrm{HCO}^{+} \mathrm{CH}_{4}$

$\mathrm{CH}_{5}{ }^{+} \mathrm{M}$

$\mathrm{CH}_{3} \quad \mathrm{H}$

$\begin{array}{ll}\mathrm{CH}_{2} & \mathrm{H}_{2}\end{array}$

$\mathrm{CH}_{2} \quad \mathrm{H} \quad \mathrm{H}$

$\mathrm{CH} \quad \mathrm{H}_{2} \quad \mathrm{H}$

$\mathrm{CH} \quad \mathrm{H} \quad \mathrm{H}$

$\mathrm{H}_{3} \mathrm{O}^{+} \mathrm{CH}_{3}$

$\mathrm{CO}_{2} \mathrm{H}^{+} \mathrm{CH}_{3}$

$\mathrm{CH}_{5}{ }^{+} \mathrm{NH}_{2}$

$\mathrm{NH}_{4}{ }^{+} \mathrm{CH}_{3}$
$\frac{A}{7.20 E-10}$

$\frac{B}{0.0}$

C

1.00E-09

0.0

7. $28 \mathrm{E}-10$

0.0

1. 82E-10

0.0

2. 53E-07

$-0.5$

2.54E-07 -0.5

$2.53 E-07-0.5$

$3.20 \mathrm{E}-10 \quad 0.0$

$1.28 \mathrm{E}-09 \quad 0.0$

$9.60 \mathrm{E}-10 \quad 0.0$

$1.00 \mathrm{E}-09 \quad 0.0$

3. 35E-11 0.0

3. 35E-11 0.0

4. $40 \mathrm{E}-10 \quad 0.0$

4. 30E-11 0.0

$9.40 \mathrm{E}-10 \quad 0.0$

$1.15 \mathrm{E}-09 \quad 0.0$

3. $50 \mathrm{E}-10 \quad 0.0$

4. $60 \mathrm{E}-11 \quad 0.0$

$5.24 \mathrm{E}-10 \quad 0.0$

$1.48 \mathrm{E}-09 \quad 0.0$

$1.57 \mathrm{E}-10 \quad 0.0$

$1.04 \mathrm{E}-10 \quad 0.0$

$1.00 \mathrm{E}-09 \quad 0.0$

$1.30 \mathrm{E}-28 \quad 0.0$

$1.20 \mathrm{E}-07-0.5$

$1.20 \mathrm{E}-07-0.5$

$1.20 \mathrm{E}-07-0.5$

$1.20 \mathrm{E}-07-0.5$

H

$1.20 \mathrm{E}-07-0.5$

$3.50 \mathrm{E}-090.0$

$1.20 \mathrm{E}-09 \quad 0.0$

$6.40 E-110.0$

$1.54 E-09 \quad 0.0$
Refs. and Notes

0.010

0.0 a

$0.0 \quad 42, e$

$0.0 \quad 42, e$

0.052

$0.0 \quad 52$

0.052

$0.0 \quad 10,39, f$

$0.0 \quad 10,39, f$

0.010

0.0 a

$0.010,16$, a

$0.010,16, a$

$0.010,16$

0.010

0.010

0.010

0.010

0.010

$0.0 \quad 10$

0.010

0.010

0.010

0.010

$0.0 \quad 17, a$

$0.0 \quad 52$

0.052

0.052

$0.0 \quad 52$

0.052

0.010

0.038

0.010

0.010 
Table IV (cont)

\begin{tabular}{|c|c|c|c|c|c|c|c|c|c|c|}
\hline \multicolumn{2}{|c|}{ Reactants } & & \multicolumn{3}{|c|}{ Products } & \multirow{2}{*}{$\frac{A}{1.60 E-09}$} & \multirow{2}{*}{$\frac{B}{0.0}$} & \multirow{2}{*}{$\frac{c}{0.0}$} & \multirow{2}{*}{$\frac{\operatorname{Refs} \text {. and }}{10}$} & Notes \\
\hline $\mathrm{CH}_{4}^{+}$ & $\mathrm{NH}_{3}$ & & $\mathrm{NH}_{3}{ }^{+}$ & $\mathrm{CH}_{4}$ & & & & & & \\
\hline $\mathrm{CH}_{4}^{+}$ & $\mathrm{CH}_{4}$ & & $\mathrm{CH}_{5}{ }^{+}$ & $\mathrm{CH}_{3}$ & & $1.15 E-09$ & 0.0 & 0.0 & 10 & \\
\hline $\mathrm{CH}_{4}^{+}$ & $\mathrm{H}_{2}$ & & $\mathrm{CH}_{5}{ }^{+}$ & $\mathrm{H}$ & & $4.10 E-11$ & 0.0 & 0.0 & 10 & \\
\hline $\mathrm{CH}_{4}^{+}$ & $0^{2}$ & & $\mathrm{H}_{2} \mathrm{CO}^{+}$ & $\mathrm{H}_{2}$ & & $1.00 \mathrm{E}-09$ & 0.0 & 0.0 & $\mathbf{a}$ & \\
\hline $\mathrm{CH}_{4}^{+}$ & $\mathrm{O}_{2}$ & & $\mathrm{o}_{2}^{+}$ & $\mathrm{CH}_{4}$ & & $4.40 E-10$ & 0.0 & 0.0 & 42 & \\
\hline $\mathrm{CH}_{4}^{+}$ & $\mathrm{CO}$ & & $\mathrm{CH}_{3} \mathrm{CO}^{+}$ & $t_{H}$ & & 7. $02 \mathrm{E}-11$ & 0.0 & 0.0 & $39, \mathrm{f}$ & \\
\hline $\mathrm{CH}_{4}^{+}$ & $\mathrm{CO}$ & & $\mathrm{HCO}^{+}$ & $\mathrm{CH}_{3}$ & & $7.10 \mathrm{E}-10$ & 0.0 & 0.0 & $39, f$ & \\
\hline $\mathrm{CH}_{4}^{+}$ & $\mathrm{C}_{2} \mathrm{H}_{2}$ & & $\mathrm{C}_{2} \mathrm{H}_{2}{ }^{+}$ & $\mathrm{CH}_{4}$ & & $1.13 \mathrm{E}-09$ & 0.0 & 0.0 & 10 & \\
\hline $\mathrm{CH}_{4}^{+}$ & $\mathrm{C}_{2} \mathrm{H}_{2}$ & & $\mathrm{C}_{2} \mathrm{H}_{3}{ }^{+}$ & $\mathrm{CH}_{3}$ & & $1.24 E-09$ & 0.0 & 0.0 & 10 & \\
\hline $\mathrm{CH}_{4}^{+}$ & $\mathrm{C}_{2} \mathrm{H}_{2}$ & & $\mathrm{C}_{3} \mathrm{H}_{3}{ }^{+}$ & $\mathrm{H}_{2}$ & $\mathrm{H}$ & $1.51 \mathrm{E}-10$ & 0.0 & 0.0 & 10 & \\
\hline $\mathrm{CH}_{4}^{+}$ & $\mathrm{C}_{2} \mathrm{H}_{4}$ & & $\mathrm{C}_{2} \mathrm{H}_{4}^{+}$ & $\mathrm{CH}_{4}$ & & $1.38 \mathrm{E}-09$ & 0.0 & 0.0 & 10 & \\
\hline $\mathrm{CH}_{4}^{+}$ & $\mathrm{C}_{2} \mathrm{H}_{4}$ & & $\mathrm{c}_{2} \mathrm{H}_{5}{ }^{+}$ & $\mathrm{CH}_{3}$ & & $4 \cdot 23 \mathrm{E}-10$ & 0.0 & 0.0 & 10 & \\
\hline $\mathrm{CH}_{4}^{+}$ & $\mathrm{C}_{2} \mathrm{H}_{4}$ & & $\mathrm{C}_{3} \mathrm{H}_{5}{ }^{+}$ & $\mathrm{H}_{2}$ & $\mathbf{H}$ & $5.50 E-11$ & 0.0 & 0.0 & 10 & \\
\hline $\mathrm{CH}_{4}^{+}$ & $\mathrm{C}_{2} \mathrm{H}_{6}$ & & $\mathrm{C}_{2} \mathrm{H}_{4}^{+}$ & $\mathrm{CH}_{4}$ & $\mathrm{H}_{2}$ & $1.91 E-09$ & 0.0 & 0.0 & 10 & \\
\hline $\mathrm{CH}_{5}{ }^{+}$ & e & & $\mathrm{CH}_{4}$ & H & & $7.00 \mathrm{E}-07$ & -0.5 & 0.0 & 52 & \\
\hline $\mathrm{CH}_{5}^{+}$ & $\mathrm{H}_{2} \mathrm{O}$ & & $\mathrm{H}_{3} \mathrm{O}^{+}$ & $\mathrm{CH}_{4}$ & & $3.70 E-09$ & 0.0 & 0.0 & $\mathbf{a}$ & \\
\hline $\mathrm{CH}_{5}{ }^{+}$ & $\mathrm{NH}_{3}$ & & $\mathrm{NH}_{4}^{+}$ & $\mathrm{CH}_{4}$ & & $2.50 \mathrm{E}-09$ & 0.0 & 0.0 & a & \\
\hline $\mathrm{CH}_{5}^{+}$ & $\mathrm{CO}$ & & $\mathrm{HCO}^{+}$ & $\mathrm{CH}_{4}$ & & $1.00 \mathrm{E}-09$ & 0.0 & 0.0 & $42, a$ & \\
\hline $\mathrm{CH}_{5}{ }^{+}$ & $\mathrm{CH}_{3}$ & & $\mathrm{C}_{2} \mathrm{H}_{6}{ }^{+}$ & $\mathrm{H}_{2}$ & & $5.00 E-10$ & 0.0 & 0.0 & $17, a$ & \\
\hline $\mathrm{CH}_{5}{ }^{+}$ & $\mathrm{C}_{2} \mathrm{H}_{6}$ & & $\mathrm{C}_{2} \mathrm{H}_{7}{ }^{+}$ & $\mathrm{CH}_{4}$ & & $1.50 E-09$ & 0.0 & 0.0 & $17, a$ & \\
\hline $\mathrm{CH}_{5}{ }^{+}$ & $\mathrm{CH}_{4}$ & M & $\mathrm{C}_{2} \mathrm{H}_{9}{ }^{+}$ & M & & $1.00 E-29$ & 0.0 & 0.0 & $17, a$ & \\
\hline $\mathrm{N}^{+}$ & e & & $\mathbf{N}$ & & & 3. $50 \mathrm{E}-12$ & -0.7 & 0.0 & 11 & \\
\hline $\mathrm{N}^{+}$ & $\mathrm{H}_{2} \mathrm{O}$ & & $\mathrm{H}_{2} \mathrm{O}^{+}$ & $\mathbf{N}$ & & $2.60 E-09$ & 0.0 & 0.0 & 11 & \\
\hline $\mathrm{N}^{+}$ & $\mathrm{CO}_{2}$ & & $\mathrm{CO}_{2}^{+}$ & $\mathrm{N}$ & & $1.10 E-09$ & 0.0 & 0.0 & 10 & \\
\hline $\mathrm{N}^{+}$ & $\mathrm{CH}_{4}$ & & $\mathrm{CH}_{3}{ }^{+}$ & $\mathbf{N}$ & H & $7.16 \mathrm{E}-10$ & 0.0 & 0.0 & 10 & \\
\hline $\mathrm{N}^{+}$ & $\mathrm{CH}_{4}$ & & $\mathrm{CH}_{4}^{+}$ & $\mathbf{N}$ & & $5.40 \mathrm{E}-11$ & 0.0 & 0.0 & 10 & \\
\hline $\mathrm{N}^{+}$ & $\mathrm{CH}_{4}$ & & $\mathrm{H}_{2} \mathrm{CN}^{+}$ & H & $\mathbf{H}$ & 4. $32 E-10$ & 0.0 & 0.0 & 10 & \\
\hline $\mathrm{N}^{+}$ & $\mathrm{CH}_{4}$ & & $\mathrm{HCN}^{+}$ & $\mathrm{H}_{2}$ & H & $1.35 E-10$ & 0.0 & 0.0 & 10 & \\
\hline $\mathrm{N}^{+}$ & $\mathrm{H}_{2}$ & & $\mathrm{NH}^{+}$ & H & & $4.80 E-10$ & 0.0 & 0.0 & 10 & \\
\hline $\mathrm{N}^{+}$ & $\mathrm{co}$ & & $\mathrm{CO}^{+}$ & $\mathbf{N}$ & & $4.90 \mathrm{E}-10$ & 0.0 & 0.0 & 10 & \\
\hline $\mathrm{N}^{+}$ & $\mathrm{CO}$ & & $\mathrm{NO}^{+}$ & C & & $6.00 E-11$ & 0.0 & 0.0 & 10 & \\
\hline $\mathrm{N}^{+}$ & NH & & $\mathrm{N}_{2}^{+}$ & $\mathrm{H}$ & & $1.00 \mathrm{E}-09$ & 0.0 & 0.0 & $\mathbf{a}$ & \\
\hline $\mathrm{N}^{+}$ & $o_{2}$ & & $0^{+}$ & NO & & $3.60 \mathrm{E}-11$ & 0.0 & 0.0 & 10 & \\
\hline $\mathrm{N}^{+}$ & $\mathrm{O}_{2}$ & & $\mathrm{NO}^{+}$ & 0 & & $1.70 \mathrm{E}-10$ & 0.0 & 0.0 & 10 & \\
\hline
\end{tabular}


Table IV (cont)

\begin{tabular}{|c|c|c|c|c|c|c|c|c|c|}
\hline \multicolumn{2}{|c|}{ Reactants } & \multicolumn{3}{|c|}{ Products } & \multirow{2}{*}{$\frac{A}{4.00 E-10}$} & \multirow{2}{*}{$\frac{B}{0.0}$} & \multirow{2}{*}{$\frac{\mathrm{C}}{0.0}$} & \multirow{2}{*}{\multicolumn{2}{|c|}{ Refs. and Notes }} \\
\hline $\mathrm{N}^{+}$ & $\mathrm{O}_{2}$ & $\mathrm{O}_{2}^{+}$ & $\mathbf{N}$ & & & & & & \\
\hline $\mathrm{N}^{+}$ & NO & $\mathrm{NO}^{+}$ & $\mathrm{N}$ & & $5.20 \mathrm{E}-10$ & 0.0 & 0.0 & 10 & \\
\hline $\mathrm{N}^{+}$ & NO & $\mathrm{N}_{2}^{+}$ & 0 & & $4.50 \mathrm{E}-11$ & 0.0 & 0.0 & 10 & \\
\hline $\mathrm{N}^{+}$ & 0 & $0^{+}$ & $\mathrm{N}$ & & $1.00 E-12$ & 0.0 & 0.0 & $11, a$ & \\
\hline $\mathrm{NH}^{+}$ & e & $\mathrm{N}$ & $\mathrm{H}$ & & $1.08 \mathrm{E}-07$ & -0.5 & 0.0 & 52 & \\
\hline $\mathrm{NH}^{+}$ & $\mathrm{H}_{2} \mathrm{O}$ & $\mathrm{H}_{3} \mathrm{O}^{+}$ & $\mathbf{N}$ & & $1.40 \mathrm{E}-09$ & 0.0 & 0.0 & 39,42 & \\
\hline $\mathrm{NH}^{+}$ & $\mathrm{NH}_{3}$ & $\mathrm{NH}_{3}{ }^{+}$ & NH & & $1.05 E-09$ & 0.0 & 0.0 & $10, a$ & \\
\hline $\mathrm{NH}^{+}$ & $\mathrm{NH}_{3}$ & $\mathrm{NH}_{4}{ }^{+}$ & $\mathbf{N}$ & & $1.05 E-09$ & 0.0 & 0.0 & $10, a$ & \\
\hline $\mathrm{NH}^{+}$ & $\mathrm{H}_{2}$ & $\mathrm{NH}_{2}{ }^{+}$ & $\mathbf{H}$ & & $9.50 \mathrm{E}-10$ & 0.0 & 0.0 & 10 & \\
\hline $\mathrm{NH}_{2}^{+}$ & e & $\mathrm{NH}$ & $\mathrm{H}$ & & $3.00 E-07$ & -0.5 & 0.0 & $\mathbf{a}$ & \\
\hline $\mathrm{NH}_{2}{ }^{+}$ & $\mathrm{H}_{2} \mathrm{O}$ & $\mathrm{H}_{3} \mathrm{O}^{+}$ & NH & & $1.58 \mathrm{E}-09$ & 0.0 & 0.0 & 10 & \\
\hline $\mathrm{NH}_{2}{ }^{+}$ & $\mathrm{H}_{2} \mathrm{O}$ & $\mathrm{NH}_{4}{ }^{+}$ & 0 & & $3.40 \mathrm{E}-11$ & 0.0 & 0.0 & 10 & \\
\hline $\mathrm{NH}_{2}{ }^{+}$ & $\mathrm{H}_{2} \mathrm{O}$ & $\mathrm{NH}_{3}{ }^{+}$ & $\mathrm{OH}$ & & $1.02 \mathrm{E}-10$ & 0.0 & 0.0 & 10 & \\
\hline $\mathrm{NH}_{2}{ }^{+}$ & $\mathrm{NH}_{3}$ & $\mathrm{NH}_{4}{ }^{+}$ & NH & & $9.68 \mathrm{E}-10$ & 0.0 & 0.0 & 10 & \\
\hline $\mathrm{NH}_{2}{ }^{+}$ & $\mathrm{NH}_{3}$ & $\mathrm{NH}_{3}{ }^{+}$ & $\mathrm{NH}_{2}$ & & $1.45 E-09$ & 0.0 & 0.0 & 10 & \\
\hline $\mathrm{NH}_{2}{ }^{+}$ & $\mathrm{H}_{2}$ & $\mathrm{NH}_{3}{ }^{+}$ & $\mathrm{H}$ & & $1.20 \mathrm{E}-10$ & 0.0 & 0.0 & 10 & \\
\hline $\mathrm{NH}_{3}{ }^{+}$ & e & $\mathrm{NH}_{2}$ & H & & $1.00 \mathrm{E}-07$ & -0.5 & 0.0 & $a, 8$ & \\
\hline $\mathrm{NH}_{3}{ }^{+}$ & e & $\mathrm{NH}$ & $\mathrm{H}_{2}$ & & $1.00 E-07$ & -0.5 & 0.0 & $a, 8$ & \\
\hline $\mathrm{NH}_{3}{ }^{+}$ & e & $\mathrm{NH}$ & $\mathrm{H}$ & $\mathbf{H}$ & $1.90 \mathrm{E}-07$ & -0.5 & 0.0 & $13, a, h$ & \\
\hline $\mathrm{NH}_{3}{ }^{+}$ & $\mathrm{H}_{2} \mathrm{O}$ & $\mathrm{NH}_{4}^{+}$ & $\mathrm{OH}$ & & $1.10 \mathrm{E}-10$ & 0.0 & 0.0 & .10 & \\
\hline $\mathrm{NH}_{3}{ }^{+}$ & $\mathrm{NH}_{3}$ & $\mathrm{NH}_{4}{ }^{+}$ & $\mathrm{NH}_{2}$ & & $2.30 \mathrm{E}-09$ & 0.0 & 0.0 & 10 & \\
\hline $\mathrm{NH}_{3}{ }^{+}$ & $\mathrm{CH}_{4}$ & $\mathrm{NH}_{4}{ }^{+}$ & $\mathrm{CH}_{3}$ & & $3.90 \mathrm{E}-10$ & 0.0 & 0.0 & 10 & \\
\hline $\mathrm{NH}_{3}{ }^{+}$ & $\mathrm{OH}$ & $\mathrm{NH}_{4}^{+}$ & 0 & & $1.00 E-09$ & 0.0 & 0.0 & $\mathbf{a}$ & \\
\hline $\mathrm{NH}_{3}{ }^{+}$ & $\mathrm{H}_{2}$ & $\mathrm{NH}_{4}{ }^{+}$ & $\mathrm{H}$ & & $1.70 E-11$ & 0.0 & 1043.1 & 10,22 & \\
\hline $\mathrm{NH}_{3}{ }^{+}$ & $\mathrm{H}_{2} \mathrm{CO}$ & $\mathrm{NH}_{4}{ }^{+}$ & $\mathrm{HCO}$ & & $4.90 \mathrm{E}-10$ & 0.0 & 0.0 & 10 & \\
\hline $\mathrm{NH}_{3}^{+}$ & $\mathrm{CH}_{2}$ & $\mathrm{CH}_{3}{ }^{+}$ & $\mathrm{NH}_{2}$ & & $1.00 E-09$ & 0.0 & 0.0 & a & \\
\hline $\mathrm{NH}_{4}^{+}$ & e & $\mathrm{NH}_{3}$ & $\mathrm{H}$ & & $1.17 \mathrm{E}-06$ & -0.5 & 0.0 & $11,13, a$ & \\
\hline $\mathrm{NH}_{4}^{+}$ & e & $\mathrm{NH}_{2}$ & $\mathrm{H}$ & $\mathbf{H}$ & $1.30 E-07$ & -0.5 & 0.0 & $11,13, a$ & \\
\hline $0^{+}$ & e & 0 & & & $3.50 \mathrm{E}-12$ & -0.7 & 0.0 & 11 & \\
\hline $0^{+}$ & $\mathrm{H}_{2} \mathrm{O}$ & $\mathrm{H}_{2} \mathrm{O}^{+}$ & 0 & & $2.30 E-09$ & 0.0 & 0.0 & 11 & \\
\hline $0^{+}$ & $\mathrm{CO}_{2}$ & $\mathrm{o}_{2}^{+}$ & $\mathrm{CO}$ & & $1.10 E-09$ & -0.3 & 0.0 & 11 & \\
\hline $0^{+}$ & $\mathrm{NH}_{3}$ & $\mathrm{NH}_{3}{ }^{+}$ & 0 & & $1.20 \mathrm{E}-09$ & $0: 0$ & 0.0 & 41 & \\
\hline $0^{+}$ & $\mathrm{CH}_{4}$ & $\mathrm{CH}_{4}^{+}$ & 0 &. & $8.90 \mathrm{E}-10$ & 0.0 & 0.0 & 41 & \\
\hline $0^{+}$ & $\mathrm{CH}_{4}$ & $\mathrm{CH}_{3}{ }^{+}$ & $\mathrm{OH}$ & & 1. $10 \mathrm{E}-10$ & 0.0 & 0.0 & 41 & \\
\hline
\end{tabular}


Table IV (cont)

\begin{tabular}{|c|c|c|c|c|c|c|c|c|}
\hline \multicolumn{2}{|c|}{ Reactants } & \multicolumn{2}{|c|}{ Products } & \multirow{2}{*}{$\frac{A}{1.58 \mathrm{E}-09}$} & \multirow{2}{*}{$\frac{B}{0.0}$} & \multirow{2}{*}{$\frac{\mathrm{C}}{0.0}$} & \multirow{2}{*}{$\frac{\text { Refs. and }}{10}$} & \multirow{2}{*}{ Notes } \\
\hline $0^{+}$ & $\mathrm{H}_{2}$ & $\mathrm{OH}^{+}$ & H & & & & & \\
\hline $0^{+}$ & $\mathrm{OH}$ & $\mathrm{OH}^{+}$ & 0 & $3.00 \mathrm{E}-10$ & 0.0 & 0.0 & $a$ & \\
\hline $0^{+}$ & $\mathrm{OH}$ & $\mathrm{H}^{+}$ & $\mathrm{O}_{2}$ & $2.70 \mathrm{E}-11$ & 0.13 & 0.0 & $a$ & \\
\hline $0^{+}$ & $\mathrm{O}_{2}$ & $\mathrm{O}_{2}^{+}$ & 0 & $2.00 \mathrm{E}-11$ & -0.5 & 0.0 & 11 & \\
\hline $0^{+}$ & $\mathrm{N}_{2}$ & $\mathrm{NO}^{+}$ & $\mathbf{N}$ & $1.20 E-12$ & $-1 \cdot 0$ & 0.0 & 11 & \\
\hline $0^{+}$ & $\mathrm{H}$ & $\mathrm{H}^{+}$ & 0 & $6.80 \mathrm{E}-10$ & 0.0 & 0.0 & 11 & \\
\hline $\mathrm{OH}^{+}$ & e & 0 & $\mathbf{H}$ & $8.22 E-08$ & -0.5 & 0.0 & 52 & \\
\hline $\mathrm{OH}^{+}$ & $\mathrm{H}_{2} \mathrm{O}$ & $\mathrm{H}_{3} \mathrm{O}^{+}$ & 0 & $1.30 \mathrm{E}-09$ & 0.0 & 0.0 & 10 & \\
\hline $\mathrm{OH}^{+}$ & $\mathrm{H}_{2} \mathrm{O}$ & $\mathrm{H}_{2} \mathrm{O}^{+}$ & $\mathrm{OH}$ & $1.59 \mathrm{E}-09$ & 0.0 & 0.0 & 10 & \\
\hline $\mathrm{OH}^{+}$ & $\mathrm{NH}_{3}$ & $\mathrm{NH}_{4}{ }^{+}$ & 0 & $1.19 E-09$ & 0.0 & 0.0 & 10 & \\
\hline $\mathrm{OH}^{+}$ & $\mathrm{NH}_{3}$ & $\mathrm{NH}_{3}{ }^{+}$ & $\mathrm{OH}$ & $1.19 \mathrm{E}-09$ & 0.0 & 0.0 & 10 & \\
\hline $\mathrm{OH}^{+}$ & $\mathrm{CH}_{4}$ & $\mathrm{H}_{3} \mathrm{O}^{+}$ & $\mathrm{CH}_{2}$ & $1.32 \mathrm{E}-10$ & 0.0 & 0.0 & $39, f$ & \\
\hline $\mathrm{OH}^{+}$ & $\mathrm{CH}_{4}$ & $\mathrm{CH}_{4}^{+}$ & $\mathrm{OH}$ & $9.68 \mathrm{E}-10$ & 0.0 & 0.0 & $39, f$ & \\
\hline $\mathrm{OH}^{+}$ & $\mathrm{H}_{2} \mathrm{CO}$ & $\mathrm{CH}_{2} \mathrm{Ol}$ & ${ }^{+} 0$ & $1.12 E-09$ & 0.0 & 0.0 & 15 & \\
\hline $\mathrm{OH}^{+}$ & $\mathrm{H}_{2} \mathrm{CO}$ & $\mathrm{H}_{2} \mathrm{CO}^{-1}$ & $\mathrm{OH}$ & $7.44 \mathrm{E}-10$ & 0.0 & 0.0 & 15 & \\
\hline $\mathrm{OH}^{+}$ & $\mathrm{CO}_{2}$ & $\mathrm{CO}_{2} \mathrm{H}^{-}$ & 0 & $1.10 \mathrm{E}-09$ & 0.0 & 0.0 & $39, f$ & \\
\hline $\mathrm{OH}^{+}$ & $\mathrm{HCN}$ & $\mathrm{H}_{2} \mathrm{CN}^{-1}$ & 0 & $1.00 \mathrm{E}-09$ & 0.0 & 0.0 & $a$ & \\
\hline $\mathrm{OH}^{+}$ & $\mathrm{CO}$ & $\mathrm{HCO}^{+}$ & 0 & $6.50 E-10$ & 0.0 & 0.0 & $39, f$ & \\
\hline $\mathrm{OH}^{+}$ & $\mathrm{N}_{2}$ & $\mathrm{~N}_{2} \mathrm{H}^{+}$ & 0 & $1.90 \mathrm{E}-10$ & 0.0 & 0.0 & 15 & \\
\hline $\mathrm{OH}^{+}$ & $\mathrm{OH}$ & $\mathrm{H}_{2} \mathrm{O}^{+}$ & 0 & $1.00 E-09$ & 0.0 & 0.0 & $\mathbf{a}$ & \\
\hline $\mathrm{OH}^{+}$ & $\mathrm{H}_{2}$ & $\mathrm{H}_{2} \mathrm{O}^{+}$ & $\mathrm{H}$ & $1.05 \mathrm{E}-09$ & 0.0 & 0.0 & 10 & \\
\hline $\mathrm{OH}^{+}$ & $\mathrm{C}$ & $\mathrm{CO}^{+}$ & $\mathrm{H}$ & $1.00 \mathrm{E}-09$ & 0.0 & 0.0 & $14, a$ & \\
\hline $\mathrm{OH}^{+}$ & C & $\mathrm{CH}^{+}$ & 0 & $1.00 \mathrm{E}-09$ & 0.0 & 0.0 & a & \\
\hline $\mathrm{OH}^{+}$ & $\mathrm{O}_{2}$ & $\mathrm{O}_{2}^{+}$ & $\mathrm{OH}$ & $2.00 \mathrm{E}-10$ & 0.0 & 0.0 & 11 & \\
\hline $\mathrm{OH}^{+}$ & $\mathrm{C}_{2} \mathrm{H}_{5}$ & $\mathrm{C}_{2} \mathrm{H}_{6}{ }^{-}$ & 0 & $\therefore .00 E-09$ & 0.0 & 0.0 & $\mathbf{a}$ & \\
\hline $\mathrm{H}_{2} \mathrm{O}^{+}$ & e & $\mathrm{OH}$ & $\mathbf{H}$ & $5.32 \mathrm{E}-07$ & -0.5 & 0.0 & 52 & \\
\hline $\mathrm{H}_{2} \mathrm{O}^{+}$ & e & 0 & $\mathrm{H}_{2}$ & $1.52 \mathrm{E}-07$ & -0.5 & 0.0 & 52 & \\
\hline $\mathrm{H}_{2} \mathrm{O}^{+}$ & e & $\mathrm{H}_{2}$ & $v(1 D)$ & $7.60 \mathrm{E}-08$ & -0.5 & 0.0 & 52 & \\
\hline $\mathrm{H}_{2} \mathrm{O}^{+}$ & $\mathrm{H}_{2} \mathrm{O}$ & $\mathrm{H}_{3} \mathrm{O}^{+}$ & $\mathrm{OH}$ & $2.05 E-09$ & 0.0 & 0.0 & 10 & \\
\hline $\mathrm{H}_{2} \mathrm{O}^{+}$ & $\mathrm{NH}_{3}$ & $\mathrm{NH}_{3}{ }^{+}$ & $\mathrm{H}_{2} \mathrm{O}$ & $2.20 E-09$ & 0.0 & 0.0 & 10 & \\
\hline $\mathrm{H}_{2} \mathrm{O}^{+}$ & $\mathrm{NH}_{3}$ & $\mathrm{NH}_{4}^{+}$ & $\mathrm{OH}$ & $9.45 E-10$ & 0.0 & 0.0 & 10 & \\
\hline $\mathrm{H}_{2} \mathrm{O}^{+}$ & $\mathrm{CH}_{4}$ & $\mathrm{H}_{3} \mathrm{O}^{+}$ & $\mathrm{CH}_{3}$ & $1.10 E-09$ & 0.0 & 0.0 & 10 & \\
\hline $\mathrm{H}_{2} \mathrm{O}^{+}$ & $\mathrm{H}_{2} \mathrm{CO}$ & $\mathrm{CH}_{2} \mathrm{OH}$ & OH & $6.62 \mathrm{E}-10$ & 0.0 & 0.0 & 15 & \\
\hline $\mathrm{H}_{2} \mathrm{O}^{+}$ & $\mathrm{H}_{2} \mathrm{CO}$ & $\mathrm{H}_{2} \mathrm{CO}^{-1}$ & $\mathrm{H}_{2} \mathrm{O}$ & $1.41 E-09$ & 0.0 & 0.0 & 15 & \\
\hline
\end{tabular}


Table IV (cont)

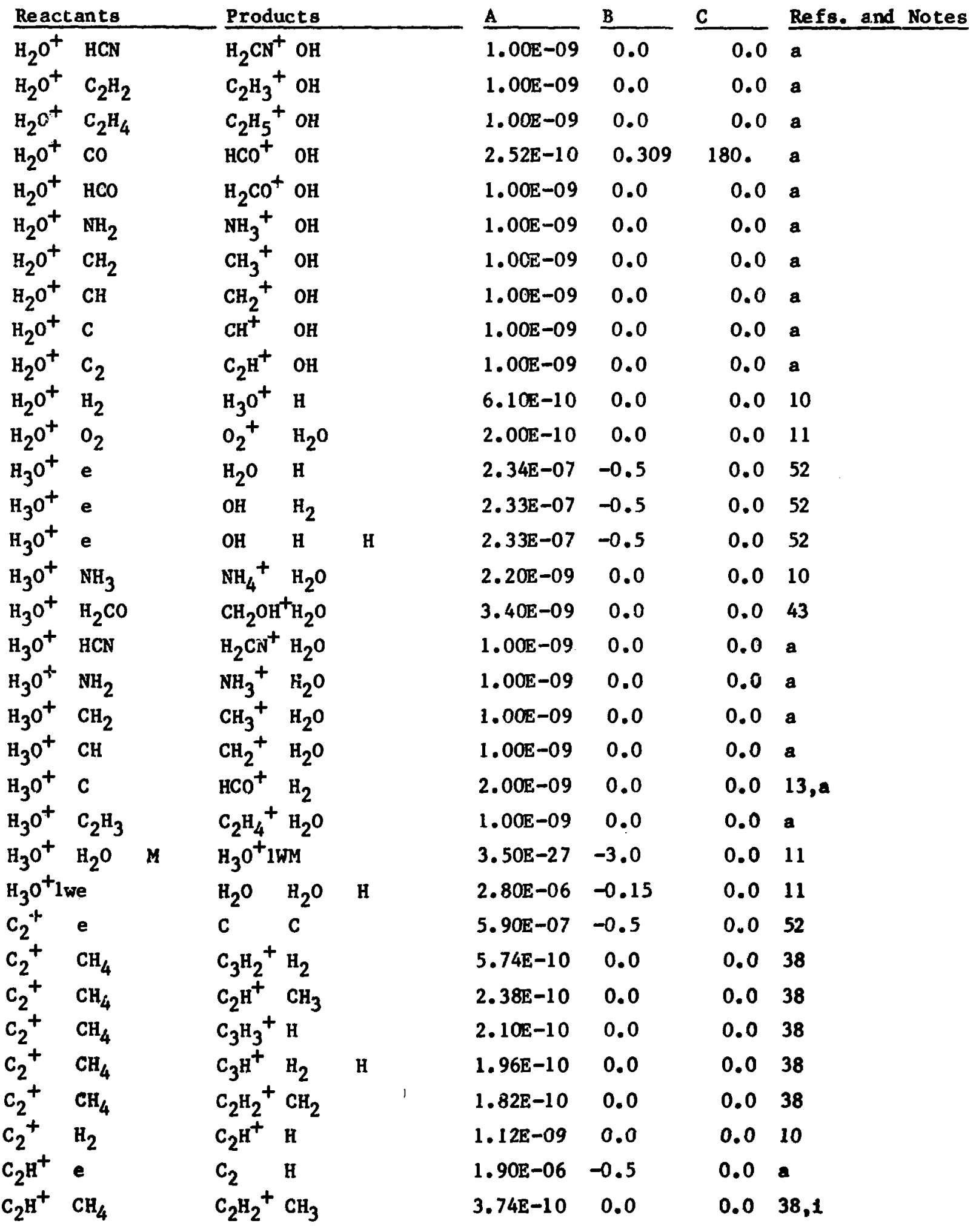


Table IV (cont)

\begin{tabular}{|c|c|c|c|c|c|c|c|c|c|}
\hline \multicolumn{3}{|c|}{ Reactants } & \multicolumn{3}{|c|}{ Products } & \multirow{2}{*}{$\frac{\mathrm{A}}{3.74 \mathrm{E}-10}$} & \multirow{2}{*}{$\frac{B}{0.0}$} & \multirow{2}{*}{$\frac{\mathrm{C}}{0.0}$} & \multirow{2}{*}{$\frac{\operatorname{Refs} . \text { and }}{38,1}$} \\
\hline $\mathrm{C}_{2} \mathrm{H}^{+}$ & $\mathrm{CH}_{4}$ & & $\mathrm{C}_{3} \mathrm{H}_{3}{ }^{+}$ & $\mathrm{H}_{2}$ & & & & & \\
\hline $\mathrm{C}_{2} \mathrm{H}^{+}$ & $\mathrm{CH}_{4}$ & & $\mathrm{c}_{3} \mathrm{H}_{4}{ }^{+}$ & $\mathrm{H}$ & & $1.32 \mathrm{E}-10$ & 0.0 & 0.0 & 38,1 \\
\hline $\mathrm{C}_{2} \mathrm{H}^{+}$ & $\mathrm{NH}_{3}$ & & $\mathrm{NH}_{4}^{+}$ & $c_{2}$ & & $1.00 \mathrm{E}-09$ & 0.0 & 0.0 & a \\
\hline $\mathrm{C}_{2} \mathrm{H}^{+}$ & $\mathrm{H}_{2} \mathrm{CO}$ & & $\mathrm{CH}_{2} \mathrm{OH}^{+}$ & ${ }^{+} \mathrm{C}_{2}$ & & $1.00 \mathrm{E}-09$ & 0.0 & 0.0 & $\mathbf{a}$ \\
\hline $\mathrm{C}_{2} \mathrm{H}^{+}$ & $\mathrm{HCN}$ & & $\mathrm{H}_{2} \mathrm{CN}^{+}$ & $c_{2}$ & & $1.00 \mathrm{E}-09$ & 0.0 & 0.0 & $\mathrm{a}$ \\
\hline $\mathrm{C}_{2} \mathrm{H}^{+}$ & $\mathrm{H}_{2}$ & & $\mathrm{C}_{2} \mathrm{H}_{2}{ }^{+}$ & $\mathrm{H}$ & & $7.80 \mathrm{E}-10$ & 0.0 & 0.0 & 10 \\
\hline $\mathrm{C}_{2} \mathrm{H}_{2}{ }^{+}$ & e & & $\mathrm{C}_{2} \mathrm{H}$ & H & & $2.95 E-07$ & -0.5 & 0.0 & 52 \\
\hline $\mathrm{C}_{2} \mathrm{H}_{2}{ }^{+}$ & e & & $c_{2}$ & $\mathbf{H}$ & $\mathrm{H}$ & $2.95 E-07$ & -0.5 & 0.0 & 52 \\
\hline $\mathrm{C}_{2} \mathrm{H}_{2}{ }^{+}$ & $\mathrm{H}_{2} \mathrm{O}$ & & $\mathrm{H}_{3} \mathrm{O}^{+}$ & $\mathrm{C}_{2} \mathrm{H}$ & & $2.20 \mathrm{E}-10$ & 0.0 & 0.0 & 10 \\
\hline $\mathrm{C}_{2} \mathrm{H}_{2}{ }^{+}$ & $\mathrm{NH}_{3}$ & & $\mathrm{NH}_{3}{ }^{+}$ & $\mathrm{C}_{2} \mathrm{H}_{2}$ & & $2.14 E-09$ & 0.0 & 0.0 & 10 \\
\hline $\mathrm{C}_{2} \mathrm{H}_{2}{ }^{+}$ & $\mathrm{NH}_{3}$ & & $\mathrm{NH}_{4}^{+}$ & $\mathrm{C}_{2} \mathrm{H}$ & & $9.61 \mathrm{E}-10$ & 0.0 & 0.0 & 10 \\
\hline $\mathrm{C}_{2} \mathrm{H}_{2}{ }^{+}$ & $\mathrm{CH}_{4}$ & & $\mathrm{C}_{3} \mathrm{H}_{4}{ }^{+}$ & $\mathrm{H}_{2}$ & & $1.76 \mathrm{E}-10$ & 0.0 & 0.0 & 10 \\
\hline $\mathrm{C}_{2} \mathrm{H}_{2}^{+}$ & $\mathrm{CH}_{4}$ & & $\mathrm{C}_{3} \mathrm{H}_{5}{ }^{+}$ & $\mathrm{H}$ & & $6.64 E-10$ & 0.0 & 0.0 & 10 \\
\hline $\mathrm{C}_{2} \mathrm{H}_{2}{ }^{+}$ & $\mathrm{C}_{2} \mathrm{H}_{2}$ & & $\mathrm{C}_{4} \mathrm{H}_{2}{ }^{+}$ & $\mathrm{H}_{2}$ & & $5.22 \mathrm{E}-10$ & 0.0 & 0.0 & 10 \\
\hline $\mathrm{C}_{2} \mathrm{H}_{2}{ }^{+}$ & $\mathrm{C}_{2} \mathrm{H}_{2}$ & & $\mathrm{C}_{4} \mathrm{H}_{3}{ }^{+}$ & $\mathrm{H}$ & & $8.88 E-10$ & 0.0 & 0.0 & 10 \\
\hline $\mathrm{C}_{2} \mathrm{H}_{2}{ }^{+}$ & $\mathrm{C}_{2} \mathrm{H}_{4}$ & & $\mathrm{C}_{2} \mathrm{H}_{4}{ }^{+}$ & $\mathrm{C}_{2} \mathrm{H}_{2}$ & & $4.01 E-10$ & 0.0 & 0.0 & 10 \\
\hline $\mathrm{C}_{2} \mathrm{H}_{2}^{+}$ & $\mathrm{C}_{2} \mathrm{H}_{4}$ & & $\mathrm{C}_{3} \mathrm{H}_{3}{ }^{+}$ & $\mathrm{CH}_{3}$ & & $5.07 \mathrm{E}-10$ & 0.0 & 0.0 & 10 \\
\hline $\mathrm{C}_{2} \mathrm{H}_{2}{ }^{+}$ & $\mathrm{C}_{2} \mathrm{H}_{4}$ & & $\mathrm{C}_{4} \mathrm{H}_{5}{ }^{+}$ & H & & $2.71 E-10$ & 0.0 & 0.0 & 10 \\
\hline $\mathrm{C}_{2} \mathrm{H}_{2}^{+}$ & $\mathrm{C}_{2} \mathrm{H}_{6}$ & & $\mathrm{C}_{2} \mathrm{H}_{4}^{+}$ & $\mathrm{C}_{2} \mathrm{H}_{4}$ & & $2.63 \mathrm{E}-10$ & 0.0 & 0.0 & 10 \\
\hline $\mathrm{C}_{2} \mathrm{H}_{2}^{+}$ & $\mathrm{C}_{2} \mathrm{H}_{6}$ & & $\mathrm{C}_{2} \mathrm{H}_{5}{ }^{+}$ & $\mathrm{C}_{2} \mathrm{H}_{3}$ & & $1.31 E-10$ & 0.0 & 0.0 & 10 \\
\hline $\mathrm{C}_{2} \mathrm{H}_{2}{ }^{+}$ & $\mathrm{C}_{2} \mathrm{H}_{6}$ & & $\mathrm{C}_{3} \mathrm{H}_{3}{ }^{+}$ & $\mathrm{CH}_{3}$ & $\mathrm{H}_{2}$ & $8.80 \mathrm{E}-11$ & 0.0 & 0.0 & 10 \\
\hline $\mathrm{C}_{2} \mathrm{H}_{2}^{+}$ & $\mathrm{C}_{2} \mathrm{H}_{6}$ & & $\mathrm{C}_{3} \mathrm{H}_{5}{ }^{+}$ & $\mathrm{CH}_{3}$ & & $7.88 \mathrm{E}-10$ & 0.0 & 0.0 & 10 \\
\hline $\mathrm{C}_{2} \mathrm{H}_{2}^{+}$ & $\mathrm{C}_{2} \mathrm{H}_{6}$ & & $\mathrm{C}_{4} \mathrm{H}_{5}{ }^{+}$ & $\mathrm{H}_{2}$ & $\mathrm{H}$ & $7.30 E-11$ & 0.0 & 0.0 & 10 \\
\hline $\mathrm{C}_{2} \mathrm{H}_{2}^{+}$ & $\mathrm{C}_{2} \mathrm{H}_{6}$ & & $\mathrm{C}_{4} \mathrm{H}_{7}{ }^{+}$ & H & & $1.31 E-10$ & 0.0 & 0.0 & 10 \\
\hline $\mathrm{C}_{2} \mathrm{H}_{2}^{+}$ & $\mathrm{H}_{2} \mathrm{CO}$ & & $\mathrm{HCO}^{+}$ & $\mathrm{C}_{2} \mathrm{H}_{3}$ & & $5.38 \mathrm{E}-10$ & 0.0 & 0.0 & 10 \\
\hline $\mathrm{C}_{2} \mathrm{H}_{2}^{+}$ & $\mathrm{H}_{2} \mathrm{CO}$ & & $\mathrm{H}_{2} \mathrm{CO}^{+}$ & $\mathrm{C}_{2} \mathrm{H}_{2}$ & & $8.60 E-10$ & 0.0 & 0.0 & 10 \\
\hline $\mathrm{C}_{2} \mathrm{H}_{2}^{+}$ & $\mathrm{H}_{2} \mathrm{CO}$ & & $\mathrm{CH}_{2} \mathrm{OH}^{+}$ & ${ }^{+} \mathrm{C}_{2} \mathrm{H}$ & & $4.09 E-10$ & 0.0 & 0.0 & 10 \\
\hline $\mathrm{C}_{2} \mathrm{H}_{2}^{+}$ & $\mathrm{H}_{2} \mathrm{CO}$ & & $\mathrm{C}_{3} \mathrm{H}_{3} \mathrm{O}^{+}$ & & & $6.50 \mathrm{E}-11$ & 0.0 & 0.0 & 10 \\
\hline $\mathrm{C}_{2} \mathrm{H}_{2}^{+}$ & $\mathrm{H}_{2} \mathrm{CO}$ & & $\mathrm{C}_{2} \mathrm{H}_{4}{ }^{+}$ & $\mathrm{CO}$ & & $2.80 E-10$ & 0.0 & 0.0 & 10 \\
\hline $\mathrm{C}_{2} \mathrm{H}_{2}^{+}$ & $\mathrm{HCN}$ & & $\mathrm{H}_{2} \mathrm{CN}^{+}$ & $\mathrm{C}_{2} \mathrm{H}$ & & 2. $20 E-11$ & 0.0 & 0.0 & 10 \\
\hline $\mathrm{C}_{2} \mathrm{H}_{2}{ }^{+}$ & $\mathrm{HCN}$ & & $\mathrm{C}_{3} \mathrm{H}_{2} \mathrm{~N}^{+}$ & & & 3. $1 \mathrm{GE}-11$ & 0.0 & 0.0 & 10 \\
\hline $\mathrm{C}_{2} \mathrm{H}_{2}^{+}$ & & $M$ & $\mathrm{C}_{2^{\mathrm{H}_{4}}}{ }^{+}$ & $M$ & & $1.20 E-27$ & 0.0 & 0.0 & $17, a$ \\
\hline $\mathrm{C}_{2} \mathrm{H}_{3}^{+}$ & e & & $\mathrm{C}_{2} \mathrm{H}_{2}$ & $\mathrm{H}$ & & $9.50 E-07$ & -0.5 & 0.0 & 52 \\
\hline $\mathrm{C}_{2} \mathrm{H}_{3}{ }^{+}$ & $\mathrm{H}_{2} \mathrm{O}$ & & $\mathrm{H}_{3} \mathrm{O}^{+}$ & $\mathrm{C}_{2} \mathrm{H}_{2}$ & & $1.11 E-09$ & 0.0 & 0.0 & 10 \\
\hline
\end{tabular}


Table IV (cont)

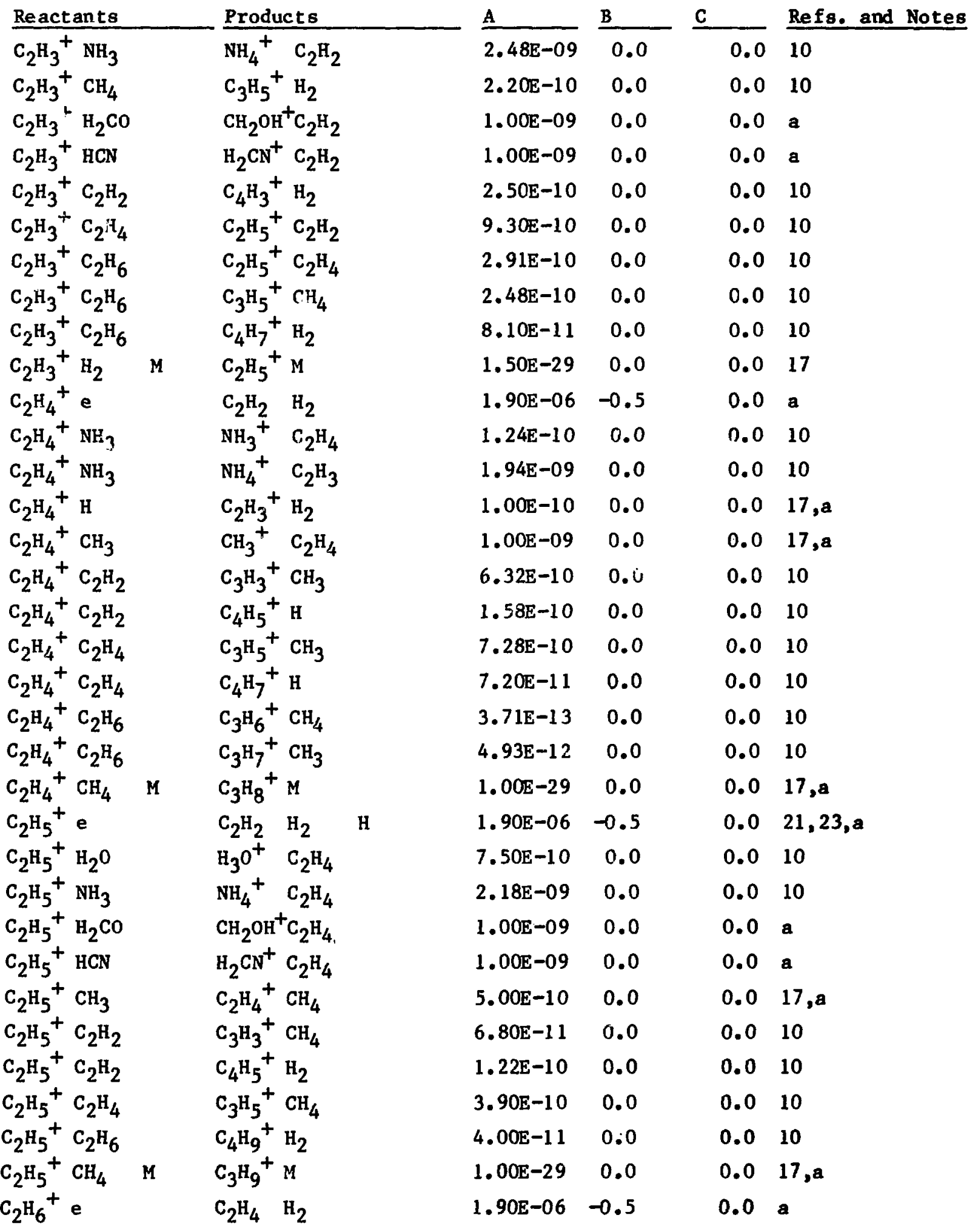


Table IV (cont)

\begin{tabular}{|c|c|c|c|c|c|c|c|c|c|c|c|}
\hline \multicolumn{3}{|c|}{ Reactants } & \multicolumn{4}{|c|}{ Products } & \multirow{2}{*}{$\frac{A}{2.95 E-09}$} & \multirow{2}{*}{$\frac{B}{0.0}$} & \multirow{2}{*}{$\frac{\mathrm{C}}{0.0}$} & \multirow{2}{*}{$\frac{\text { Refs. and }}{10}$} & Notes \\
\hline $\mathrm{C}_{2} \mathrm{H}_{6}{ }^{+}$ & $\mathrm{H}_{2} \mathrm{O}$ & & $\mathrm{H}_{3} \mathrm{O}^{+}$ & $\mathrm{C}_{2} \mathrm{H}_{5}$ & & & & & & & \\
\hline $\mathrm{C}_{2} \mathrm{H}_{6}{ }^{+}$ & $\mathrm{NH}_{3}$ & & $\mathrm{NH}_{3}{ }^{+}$ & $\mathrm{C}_{2} \mathrm{H}_{6}$ & & & $6.24 E-10$ & 0.0 & 0.0 & 10 & \\
\hline $\mathrm{C}_{2} \mathrm{H}_{6}{ }^{+}$ & $\mathrm{NH}_{3}$ & & $\mathrm{NH}_{4}^{+}$ & $\mathrm{C}_{2} \mathrm{H}_{5}$ & & & $1.61 E-09$ & 0.0 & 0.0 & 10 & \\
\hline & $\mathrm{H}_{2} \mathrm{CO}$ & & $\mathrm{CH}_{2} \mathrm{OH}$ & ${ }^{+} \mathrm{C}_{2} \mathrm{H}_{5}$ & & & $1.00 E-09$ & 0.0 & 0.0 & $\mathbf{a}$ & \\
\hline $\mathrm{C}_{2} \mathrm{H}_{6}{ }^{+}$ & $\mathrm{HCN}$ & & $\mathrm{H}_{2} \mathrm{CN}^{+}$ & $\mathrm{C}_{2} \mathrm{H}_{5}$ & & & $1,00 E-09$ & 0.0 & 0.0 & a & \\
\hline & $\mathrm{CH}_{3}$ & & $\mathrm{CH}_{3}^{+}$ & $\mathrm{C}_{2} \mathrm{H}_{6}$ & & & $1.00 E-09$ & 0.0 & 0.0 & $17, a$ & \\
\hline & $\mathrm{H}$ & & $\mathrm{C}_{2} \mathrm{H}_{5}{ }^{+}$ & $\mathrm{H}_{2}$ & & & $1.00 \mathrm{E}-10$ & 0.0 & 0.0 & $17, a$ & \\
\hline & $\mathrm{C}_{2} \mathrm{H}_{2}$ & & $\mathrm{C}_{2} \mathrm{H}_{5}{ }^{+}$ & $\mathrm{C}_{2} \mathrm{H}_{3}$ & & & $2.22 \mathrm{E}-10$ & 0.0 & 0.0 & 10 & \\
\hline & $\mathrm{C}_{2} \mathrm{H}_{2}$ & & $\mathrm{C}_{3} \mathrm{H}_{5}{ }^{+}$ & $\mathrm{CH}_{3}$ & & & $8.19 \mathrm{E}-10$ & 0.0 & 0.0 & 10 & \\
\hline & $\mathrm{C}_{2} \mathrm{H}_{2}$ & & $\mathrm{C}_{4} \mathrm{H}_{7}^{+}$ & $\mathrm{H}$ & & & $1.29 \mathrm{E}-10$ & 0.0 & 0.0 & 10 & \\
\hline $\mathrm{C}_{2}$ & $\mathrm{C}_{2} \mathrm{H}_{4}$ & & $\mathrm{C}_{2} \mathrm{H}_{4}^{+}$ & $\mathrm{C}_{2} \mathrm{H}_{6}$ & & & $1.15 E-09$ & 0.0 & 0.0 & 10 & \\
\hline & $\mathrm{C}_{2} \mathrm{H}_{6}$ & & $\mathrm{C}_{3} \mathrm{H}_{8}{ }^{+}$ & $\mathrm{CH}_{4}$ & & & $7.98 \mathrm{E}-12$ & 0.0 & 0.0 & 10 & \\
\hline & $\mathrm{C}_{2} \mathrm{H}_{6}$ & & $\mathrm{C}_{3} \mathrm{H}_{9}{ }^{+}$ & $\mathrm{CH}_{3}$ & & & $1.10 \mathrm{E}-11$ & 0.0 & 0.0 & 10 & \\
\hline & $\mathrm{CH}_{4}$ & $M$ & $\mathrm{C}_{3} \mathrm{H}_{10}$ & $t_{M}$ & & & $1.00 E-29$ & 0.0 & 0.0 & $17, a$ & \\
\hline & e & & $\mathrm{C}_{2} \mathrm{H}_{6}$ & $\mathbf{H}$ & & & $1.90 E-06$ & -0.5 & 0.0 & $a$ & \\
\hline & $\mathrm{CH}_{3}$ & & $\mathrm{C}_{2} \mathrm{H}_{6}^{+}$ & $\mathrm{CH}_{4}$ & & & $5.00 E-10$ & 0.0 & 0.0 & $17, a$ & \\
\hline & H & & $\mathrm{C}_{2} \mathrm{H}_{6}^{+}$ & $\mathrm{H}_{2}$ & & & $1.00 \mathrm{E}-10$ & 0.0 & 0.0 & $17, a$ & \\
\hline & $\mathrm{CH}_{4}$ & $\mathbf{M}$ & $\mathrm{C}_{3} \mathrm{H}_{11}$ & $+M$ & & & $1.00 \mathrm{E}-29$ & 0.0 & 0.0 & $17, \mathrm{a}$ & \\
\hline & e & & $\mathrm{C}_{2} \mathrm{H}_{6}$ & $\mathrm{H}_{2}$ & H & & $1.90 \mathrm{E}-06$ & -0.5 & 0.0 & a & \\
\hline & e & & $c_{2}$ & $\mathrm{C}$ & & & $5.00 \mathrm{E}-07$ & -0.5 & 0.0 & a & \\
\hline & $\mathrm{H}_{2}$ & & $\mathrm{C}_{3} \mathrm{H}^{+}$ & $\mathrm{H}$ & & & $1.00 \mathrm{E}-09$ & 0.0 & 0.0 & a & \\
\hline $\mathrm{C}_{3} \mathrm{H}^{+}$ & e & & $c_{3}$ & $\mathbf{H}$ & & & $1.90 \mathrm{E}-06$ & -0.5 & 0.0 & a & \\
\hline & e & & $c_{3}$ & $\mathbf{H}$ & $H$ & & $5.00 \mathrm{E}-07$ & -0.5 & 0.0 & $\mathbf{a}$ & \\
\hline & e & & $c_{3}$ & $\mathbf{H}$ & $\mathbf{H}$ & H & $5.00 E-07$ & -0.5 & 0.0 & $\mathbf{a}$ & \\
\hline & e & & $c_{3}$ & $\mathrm{H}_{2}$ & $\mathrm{H}_{2}$ & & $5.00 \mathrm{E}-07$ & -0.5 & 0.0 & $\mathbf{a}$ & \\
\hline & e & & $c_{3}$ & $\mathrm{H}_{2}$ & $\mathrm{H}_{2}$ & H & $2.50 E-07$ & -0.5 & 0.0 & a & \\
\hline & e & & $\mathrm{H}_{2} \mathrm{C}_{3} \mathrm{H}$ & & & & $2.50 \mathrm{E}-07$ & -0.5 & 0.0 & $\mathbf{a}$ & \\
\hline & e & & $\mathbf{N}$ & $\mathbf{N}$ & & & $3.60 E-07$ & -0.5 & 0.0 & 52 & \\
\hline $\mathrm{N}_{2}^{+}$ & $\mathrm{H}_{2} \mathrm{O}$ & & $\mathrm{N}_{2} \mathrm{H}^{+}$ & $\mathrm{OH}$ & & & $4.62 \mathrm{E}-10$ & 0.0 & 0.0 & $39, f$ & \\
\hline & $\mathrm{H}_{2} \mathrm{O}$ & & $\mathrm{H}_{2} \mathrm{O}^{+}$ & $\mathrm{N}_{2}$ & & & $1.64 \mathrm{E}-09$ & 0.0 & 0.0 & $39, \mathrm{f}$ & \\
\hline $\mathrm{N}_{2}^{+}$ & $\mathrm{CO}_{2}$ & & $\mathrm{CO}_{2}^{+}$ & $\mathrm{N}_{2}$ & & & $7.70 \mathrm{E}-10$ & 0.0 & 0.0 & 11 & \\
\hline $\mathrm{N}_{2}^{+}$ & $\mathrm{NH}_{3}$ & & $\mathrm{NH}_{3}{ }^{+}$ & $\mathrm{N}_{2}$ & & & $2.0 \mathrm{BE}-09$ & 0.0 & 0.0 & $39, f$ & \\
\hline $\mathrm{N}_{2}^{+}$ & $\mathrm{NH}_{3}$ & & $\mathrm{~N}_{2} \mathrm{H}^{+}$ & $\mathrm{NH}_{2}$ & & & $2.10 E-11$ & 0.0 & 0.0 & $39, f$ & \\
\hline $\mathrm{N}_{2}^{+}$ & $\mathrm{CH}_{4}$ & & $\mathrm{CH}_{4}^{+}$ & $\mathrm{N}_{2}$ & & & $1.65 E-10$ & 0.0 & 0.0 & $39, f$ & \\
\hline
\end{tabular}


Table IV (cont)

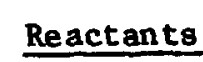

$\mathrm{N}_{2}^{+} \quad \mathrm{CH}_{4}$

$\mathrm{N}_{2}^{+} \quad \mathrm{CH}_{4}$

$\mathrm{N}_{2}{ }^{+} \quad \mathrm{H}_{2}$

$\mathrm{N}_{2}^{+} \mathrm{CO}$

$\mathrm{N}_{2}^{+} \quad 0$

$\mathrm{N}_{2}^{+} \quad 0$

$\mathrm{N}_{2}^{+} \mathrm{H}$

$\mathrm{N}_{2}^{+} \quad \mathrm{O}_{2}$

$\mathrm{N}_{2}^{+}$NO

$\mathrm{N}_{2} \mathrm{H}^{+}$e

$\mathrm{N}_{2} \mathrm{H}^{+} \quad \mathrm{H}_{2} \mathrm{O}$

$\mathrm{N}_{2} \mathrm{H}^{+} \quad \mathrm{CO}_{2}$

$\mathrm{N}_{2} \mathrm{H}^{+} \quad \mathrm{NH}_{3}$

$\mathrm{N}_{2} \mathrm{H}^{+} \quad \mathrm{CH}_{4}$

$\mathrm{N}_{2} \mathrm{H}^{+-} 60$

$\mathrm{N}_{2} \mathrm{H}^{+} \quad \mathrm{O}$

$\mathrm{O}_{2}^{+} \mathrm{e}$

$\mathrm{O}_{2}^{+}$e

$\mathrm{O}_{2}^{+} \quad \mathrm{NH}_{3}$

$\mathrm{O}_{2}^{+}$No

$\mathrm{O}_{2}^{+} \mathrm{C}$

$\mathrm{O}_{2}^{+} \mathrm{N}$

$\mathrm{HO}_{2}{ }^{+} \mathrm{e}$

$\mathrm{HO}_{2}^{+} \mathrm{H}_{2}$

$\mathrm{CO}^{+}$e

$\mathrm{CO}^{+}$e

$\mathrm{CO}^{+} \quad \mathrm{H}_{2} \mathrm{O}$

$\mathrm{CO}^{+} \quad \mathrm{H}_{2} \mathrm{O}$

$\mathrm{CO}^{+} \quad \mathrm{CO}_{2}$

$\mathrm{CO}^{+} \quad \mathrm{NH}_{3}$

$\mathrm{CO}^{+} \quad \mathrm{NH}_{3}$

$\mathrm{CO}^{+} \mathrm{CH}_{4}$

$\mathrm{CO}^{+} \mathrm{CH}_{4}$

$\mathrm{CO}^{+} \mathrm{CH}_{4}$
Products

$\mathrm{CH}_{3}{ }^{+} \mathrm{H} \quad \mathrm{N}_{2}$

$\mathrm{CH}_{2}^{+} \mathrm{H}_{2} \quad \mathrm{~N}_{2}$

$\mathrm{N}_{2} \mathrm{H}^{+} \mathrm{H}$

$\mathrm{CO}^{+} \mathrm{N}_{2}$

$\mathrm{NO}^{+} \mathrm{N}$

$\mathrm{O}^{+} \quad \mathrm{N}_{2}$

$\mathrm{H}^{+} \quad \mathrm{N}_{2}$

$\mathrm{O}_{2}^{+} \quad \mathrm{N}_{2}$

$\mathrm{NO}^{+} \mathrm{N}_{2}$

$\mathrm{N}_{2} \quad \mathrm{H}$

$\mathrm{H}_{3} \mathrm{O}^{+} \mathrm{N}_{2}$

$\mathrm{CO}_{2} \mathrm{H}^{+} \mathrm{N}_{2}$

$\mathrm{NH}_{4}^{+} \mathrm{N}_{2}$

$\mathrm{CH}_{5}{ }^{+} \mathrm{N}_{2}$

$\mathrm{HCO}^{+} \mathrm{N}_{2}$

$\mathrm{OH}^{+} \mathrm{N}_{2}$

$0 \quad 0$

$\mathrm{O}_{2}$

$\mathrm{NH}_{3}{ }^{+} \mathrm{O}_{2}$

$\mathrm{NO}^{+} \mathrm{O}_{2}$

$\mathrm{C}^{+} \mathrm{O}_{2}$

$\mathrm{NO}^{+} \mathrm{O}$

$\mathrm{O}_{2} \quad \mathrm{H}$

$\mathrm{H}_{3}{ }^{+} \mathrm{O}_{2}$

C $O(1 D)$

C(1D) 0

$\mathrm{H}_{2} \mathrm{O}^{+} \mathrm{CO}$

$\mathrm{HCO}^{+} \mathrm{OH}$

$\mathrm{CO}_{2}{ }^{+} \mathrm{CO}$

$\mathrm{NH}_{3}^{+} \mathrm{CO}$

$\mathrm{HCO}^{+} \mathrm{NH}_{2}$

$\mathrm{CH}_{4}^{+} \mathrm{CO}$

$\mathrm{HCO}^{+} \mathrm{CH}_{3}$

$\mathrm{CH}_{3} \mathrm{CO}^{+} \mathrm{H}$
A

$7.66 \mathrm{E}-10$

$3.88 \mathrm{E}-11 \quad 0.0$

$1.73 E-09 \quad 0.0$

$7.00 \mathrm{E}-11 \quad 0.0$

$1.30 \mathrm{E}-10 \quad-0.46$

$1.00 \mathrm{E}-11-0.23$

$1.20 \mathrm{E}-10 \quad 0.0$

$5.00 \mathrm{E}-11 \quad-0.8$

3. 30E-10 0.0

$7.60 \mathrm{E}-07 \quad-0.5$

$2.60 \mathrm{E}-09 \quad 0.0$

9.20₹-10 0.0

2. 30E-09 0.0

$8.90 \mathrm{E}-10 \quad 0.0$

$8.80 \mathrm{E}-10 \quad 0.0$

$1.40 \mathrm{E}-10 \quad 0.0$

$1.90 \mathrm{E}-07 \quad-0.5$

$4.00 \mathrm{E}-12 \quad-0.7$

$2.40 \mathrm{E}-09 \quad 0.0$

$4.40 \mathrm{E}-10 \quad 0.0$

$1.00 \mathrm{E}-09 \quad 0.0$

$1.80 \mathrm{E}-10 \quad 0.0$

$5.00 \mathrm{E}-07 \quad-0.5$

$3.20 \mathrm{E}-10 \quad 0.0$

$2.50 \mathrm{E}-07 \quad-0.5$

$2.50 E-07 \quad-0.5$

$1.72 \mathrm{E}-09 \quad 0.0$

8.84E-10 0.0

$1.10 E-09 \quad 0.0$

2.06E-09 0.0

$4.20 E-11 \quad 0.0$

$1.16 \mathrm{E}-09 \quad 0.0$

$2.10 \mathrm{E}-10 \quad 0.0$

$2.80 \mathrm{E}-11 \quad 0.0$
C

$0.039, f$

$0.039, f$

0.010

0.012

0.011

0.011

0.044

$0.0 \quad 11,12$

0.011

0.052

0.043

0.012

0.043

0.012

0.024

0.043

0.052

$0.011, a$

0.012

0.011

$0.013, a$

0.011

0.0 a

0.010

0.0 a

0.0 a

$0.039, f$

$0.039, f$

$0.0 \quad 45, e, j$

$0.0 \quad 39,6$

$0.039, f$

$0.039,6$

$0.039, f$

$0.039, f$ 
Table IV (cont)

\begin{tabular}{|c|c|c|c|c|}
\hline \multicolumn{2}{|c|}{ Reactants } & \multicolumn{3}{|c|}{ Products } \\
\hline $\mathrm{CO}^{+}$ & $\mathrm{H}_{2} \mathrm{CO}$ & $\mathrm{B}_{2} \mathrm{CO}^{+}$ & $\mathrm{CO}$ & \\
\hline $\mathrm{CO}^{+}$ & $\mathrm{H}_{2} \mathrm{CO}$ & $\mathrm{HCO}^{+}$ & $\mathrm{HCO}$ & \\
\hline $\mathrm{CO}^{+}$ & $\mathrm{H}_{2} \mathrm{CO}_{2}$ & $\mathrm{H}_{2} \mathrm{CO}_{2}{ }^{+}$ & ${ }^{+} \mathrm{CO}$ & \\
\hline $\mathrm{Co}^{+}$ & $\mathrm{HCN}$ & $\mathrm{HCN}^{+}$ & $\mathrm{Co}$ & \\
\hline $\mathrm{CO}^{+}$ & $\mathrm{HCN}$ & $\mathrm{HCO}^{+}$ & $\mathrm{CN}$ & \\
\hline $\mathrm{CO}^{+}$ & $\mathrm{H}_{2}$ & $\mathrm{HCO}^{+}$ & $\mathrm{H}$ & \\
\hline $\mathrm{CO}^{+}$ & $\mathrm{OH}$ & $\mathrm{OH}^{+}$ & Co & \\
\hline $\mathrm{CO}^{+}$ & NO & $\mathrm{NO}^{+}$ & Co & \\
\hline $\mathrm{CO}^{+}$ & $\mathrm{O}_{2}$ & $\mathrm{O}_{2}^{+}$ & CO & \\
\hline $\mathrm{CO}^{+}$ & 0 & $0^{+}$ & $\mathrm{CO}$ & \\
\hline $\mathrm{CO}^{+}$ & H & $\mathrm{H}^{+}$ & Co & \\
\hline $\mathrm{HCO}^{+}$ & e & $\mathrm{H}$ & $\mathrm{CO}$ & \\
\hline $\mathrm{HCO}^{+}$ & $\mathrm{H}_{2} \mathrm{O}$ & $\mathrm{H}_{3} \mathrm{O}^{+}$ & $\mathrm{CO}$ & \\
\hline $\mathrm{HCO}^{+}$ & $\mathrm{NH}_{3}$ & $\mathrm{NH}_{4}{ }^{+}$ & Co & \\
\hline $\mathrm{HCO}^{+}$ & $\mathrm{OH}$ & $\mathrm{CO}_{2} \mathrm{H}^{+}$ & $\mathbf{H}$ & \\
\hline $\mathrm{HCO}^{+}$ & $\mathrm{OH}$ & $\mathrm{H}_{2} \mathrm{O}^{+}$ & $\mathrm{CO}$ & \\
\hline $\mathrm{HCO}^{+}$ & $\mathrm{NH}_{2}$ & $\mathrm{NH}_{3}{ }^{+}$ & CO & \\
\hline $\mathrm{HCO}^{+}$ & $\mathrm{H}_{2} \mathrm{CO}$ & $\mathrm{CH}_{2} \mathrm{OH}^{-t}$ & ${ }^{+} \mathrm{Co}$ & \\
\hline $\mathrm{HCO}^{+}$ & $\mathrm{HCN}$ & $\mathrm{H}_{2} \mathrm{CN}^{+}$ & Co & \\
\hline $\mathrm{HCO}^{+}$ & $\mathrm{C}_{2} \mathrm{H}$ & $\mathrm{C}_{2} \mathrm{H}_{2}^{+}$ & $\mathrm{CO}$ & \\
\hline $\mathrm{HCO}^{+}$ & $\mathrm{C}_{2} \mathrm{H}_{2}$ & $\mathrm{C}_{2} \mathrm{H}_{3}{ }^{+}$ & $\mathrm{CO}$ & \\
\hline $\mathrm{HCO}^{+}$ & $\mathrm{C}_{2} \mathrm{H}_{4}$ & $\mathrm{C}_{2} \mathrm{H}_{5}{ }^{+}$ & $\mathrm{CO}$ & \\
\hline $\mathrm{HCO}^{+}$ & $\mathrm{HCO}$ & $\mathrm{H}_{2} \mathrm{CO}^{+}$ & $\mathrm{CO}$ & \\
\hline $\mathrm{HCO}^{+}$ & $\mathrm{CH}_{2}$ & $\mathrm{CH}_{3}{ }^{+}$ & $\mathrm{CO}$ & \\
\hline $\mathrm{HCO}^{+}$ & $\mathrm{CH}$ & $\mathrm{CH}_{2}{ }^{+}$ & CO & \\
\hline $\mathrm{HCO}^{+}$ & NH & $\mathrm{NH}_{2}{ }^{+}$ & $\mathrm{CO}$ & \\
\hline $\mathrm{HCO}^{+}$ & C & $\mathrm{CH}^{+}$ & $\mathrm{CO}$ & \\
\hline $\mathrm{H}_{2} \mathrm{CO}^{+}$ & e & $c 0$ & $\mathrm{H}$ & $\mathbf{H}$ \\
\hline $\mathrm{H}_{2} \mathrm{CO}_{2}{ }^{+}$ & te & Co & $\mathrm{H}$ & OHI \\
\hline $\mathrm{H}_{2} \mathrm{CO}^{+}$ & $\mathrm{H}_{2} \mathrm{O}$ & $\mathrm{H}_{3} \mathrm{O}^{+}$ & $\mathrm{HCO}$ & \\
\hline $\mathrm{H}_{2} \mathrm{CO}^{+}$ & $\mathrm{H}_{2} \mathrm{CO}$ & $\mathrm{CH}_{2} \mathrm{OH}^{+}$ & ${ }^{+} \mathrm{HCO}$ & \\
\hline $\mathrm{H}_{2} \mathrm{CO}^{+}$ & $\mathrm{NH}_{3}$ & $\mathrm{NH}_{3}{ }^{+}$ & $\mathrm{H}_{2} \mathrm{CO}$ & \\
\hline $\mathrm{H}_{2} \mathrm{CO}^{+}$ & $\mathrm{NH}_{3}$ & $\mathrm{NH}_{4}{ }^{+}$ & $\mathrm{HCO}$ & \\
\hline $\mathrm{H}_{2} \mathrm{CO}^{+}$ & $\mathrm{HCN}$ & $\mathrm{H}_{2} \mathrm{CN}^{+}$ & $\mathrm{HCO}$ & \\
\hline
\end{tabular}

$\frac{A}{2.00 E-09}$

1. $00 \mathrm{E}-09$

3. $00 \mathrm{E}-09$

1. 00E-09

$5.00 \mathrm{E}-10$

$1.39 \mathrm{E}-09$

3. $25 \mathrm{E}-10$

3. $30 \mathrm{E}-10$

2. $00 \mathrm{E}-10$

$1.40 \mathrm{E}-10$

9. $00 \mathrm{E}-11$

2. $00 \mathrm{E}-07$

2. $70 \mathrm{E}-09$

2. $60 \mathrm{E}-09$

1.00E-09

$1.00 \mathrm{E}-09$

$1.00 E-09$

1. $00 \mathrm{E}-09$

1. $14 \mathrm{E}-09$

$1.00 \mathrm{E}-09$

$1.00 \mathrm{E}-09$

$1.00 \mathrm{E}-09$

$1.00 \mathrm{E}-09$

$1.00 E-09$

$1.00 \mathrm{E}-09$

$1.00 \mathrm{E}-09$

$1.00 \mathrm{E}-09$

$1.90 \mathrm{E}-07-0.5$

$2.00 \mathrm{E}-07-0.5$

$1.60 \mathrm{E}-09$

0.0

1. $00 \mathrm{E}-09$

0.0

$1.15 \mathrm{E}-09$

0.0

1. 15E-09

0.0

$1.40 \mathrm{E}-09$

0.0
C Refs. and Notes

0.0 a

0.0 a

0.0 a

0.0 a

0.0 a

0.010

0.0 a

0.011

0.012

0.011

$0.0 \quad 37$

$0.0 \quad 13,32, a$

$0.0 \quad 40$

0.010

$0.0 \quad 13, a$

0.0 a

$0.013, a$

$0.0 \quad 13, a$

0.010

0.0 a

0.0 a

0.0 a

0.0 a

0.0 a

$0.0 \mathrm{a}$

1007. a

0.010

$0.013, \mathrm{a}, \mathrm{h}$

0.0 a

0.044

0.0 a

0.010

0.010

0.010 
Table IV (cont)

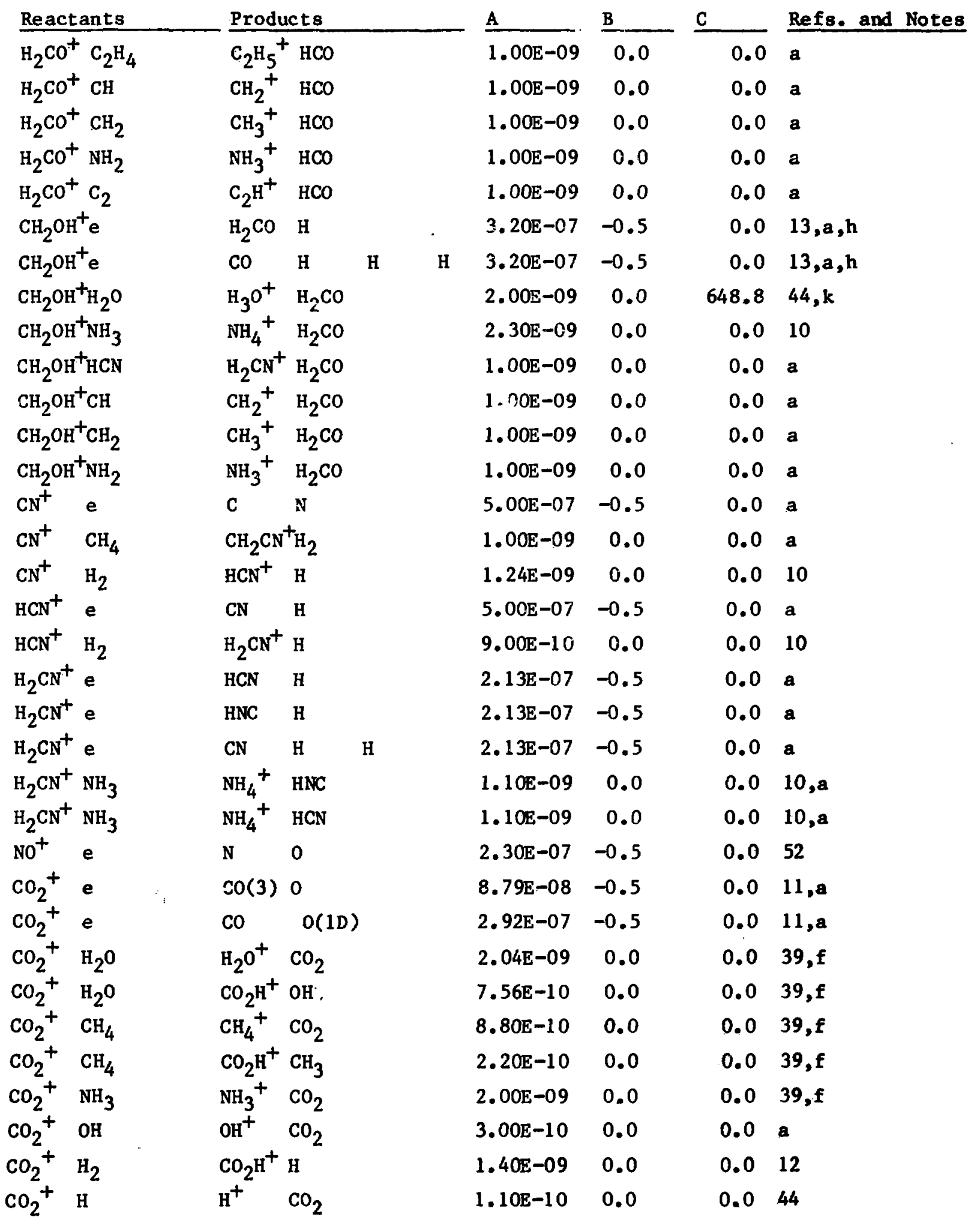


Table IV (cont)

\begin{tabular}{|c|c|c|c|c|c|c|c|c|c|}
\hline Reacta & ants & Produc & $\operatorname{cts}$ & & A & $\underline{B}$ & C & Refs. and & Notes \\
\hline $\mathrm{CO}_{2}^{+}$ & 0 & $0^{+}$ & $\mathrm{CO}_{2}$ & & $1.00 \mathrm{E}-10$ & 0.0 & 0.0 & 11 & \\
\hline $\mathrm{CO}_{2}^{+}$ & 0 & $\mathrm{O}_{2}^{+}$ & Co & & $1.60 \mathrm{E}-10$ & 0.0 & 0.0 & 11 & \\
\hline $\mathrm{CO}_{2}^{+}$ & $\mathrm{O}_{2}$ & $\mathrm{O}_{2}^{+}$ & $\mathrm{CO}_{2}$ & & $5.00 E-11$ & 0.0 & 0.0 & 11 & \\
\hline $\mathrm{CO}_{2}^{+}$ & NO & $\mathrm{NO}^{+}$ & $\mathrm{CO}_{2}$ & & $1.20 \mathrm{E}-10$ & 0.0 & 0.0 & 11 & \\
\hline $\mathrm{CO}_{2} \mathrm{H}^{+}$ & e & $\mathrm{CO}_{2}$ & $\mathbf{H}$ & & $3.20 E-07$ & -0.5 & 0.0 & $13, a, h$ & \\
\hline $\mathrm{CO}_{2} \mathrm{H}^{+}$ & e & $\mathrm{Co}$ & 0 & $\mathbf{H}$ & $3.20 \mathrm{E}-07$ & -0.5 & 0.0 & $13, a, h$ & \\
\hline $\mathrm{CO}_{2} \mathrm{H}^{+}$ & $\mathrm{H}_{2} \mathrm{O}$ & $\mathrm{H}_{3} \mathrm{O}^{+}$ & $\mathrm{CO}_{2}$ & & $3.00 E-09$ & 0.0 & 0.0 & 43 & \\
\hline $\mathrm{CO}_{2} \mathrm{H}^{+}$ & $\mathrm{NH}_{3}$ & $\mathrm{NH}_{4}^{+}$ & $\mathrm{CO}_{2}$ & & $1.00 \mathrm{E}-09$ & 0.0 & 0.0 & $43, a$ & \\
\hline $\mathrm{CO}_{2} \mathrm{H}^{+}$ & $\mathrm{CH}_{4}$ & $\mathrm{CH}_{5}^{+}$ & $\mathrm{CO}_{2}$ & & $7.80 \mathrm{E}-10$ & 0.0 & 0.0 & 43 & \\
\hline $\mathrm{CO}_{2} \mathrm{H}^{+}$ & $\mathrm{H}_{2} \mathrm{CO}$ & $\mathrm{CH}_{2} \mathrm{OH}^{-1}$ & ${ }^{+} \mathrm{CO}_{2}$ & & $1.00 E-09$ & 0.0 & 0.0 & $\mathbf{a}$ & \\
\hline $\mathrm{CO}_{2} \mathrm{H}^{+}$ & $\mathrm{HCN}$ & $\mathrm{H}_{2} \mathrm{CN}^{+}$ & $\mathrm{CO}_{2}$ & & $1.00 \mathrm{E}-09$ & 0.0 & 0.0 & $\mathbf{a}$ & \\
\hline $\mathrm{CO}_{2} \mathrm{H}^{+}$ & $\mathrm{CH}_{2}$ & $\mathrm{CH}_{3}{ }^{+}$ & $\mathrm{CO}_{2}$ & & $1.00 E-09$ & 0.0 & 0.0 & $\mathbf{a}$ & \\
\hline $\mathrm{CO}_{2} \mathrm{H}^{+}$ & $\mathrm{CO}$ & $\mathrm{HCO}^{+}$ & $\mathrm{CO}_{2}$ & & $1.00 E-09$ & 0.0 & 0.0 & $\mathbf{a}$ & \\
\hline $\mathrm{CH}_{4} \mathrm{~N}^{+}$ & e & $\mathrm{HCN}$ & $\mathrm{H}_{2}$ & $\mathbf{H}$ & $2.50 E-07$ & -0.5 & 0.0 & $a$ & \\
\hline $\mathrm{CH}_{4} \mathrm{~N}^{+}$ & e & CN & $\mathrm{H}_{2}$ & $\mathrm{H}_{2}$ & $2.50 \mathrm{E}-07$ & -0.5 & 0.0 & a & \\
\hline $\mathrm{CH}$ & $\mathrm{CH}_{4}$ & $\mathrm{C}_{2} \mathrm{H}_{4}$ & $\mathrm{H}$ & & $2.50 \mathrm{E}-12$ & 0.0 & 0.0 & 48 & \\
\hline $\mathrm{CH}_{2}$ & $\mathrm{CH}_{2}$ & $\mathrm{C}_{2} \mathrm{H}_{2}$ & $\mathrm{H}_{2}$ & & $5.30 E-11$ & 0.0 & 0.0 & 27 & \\
\hline $\mathrm{CH}_{2}$ & $\mathrm{CH}_{3}$ & $\mathrm{C}_{2} \mathrm{H}_{4}$ & $\mathbf{H}$ & & $5.00 E-11$ & 0.0 & 0.0 & $17, a$ & \\
\hline $\mathrm{C}_{2} \mathrm{H}_{3}$ & $\mathrm{H}_{2}$ & $\mathrm{C}_{2} \mathrm{H}_{4}$ & $\mathbf{H}$ & & $5.00 E-12$ & 0.0 & 3200 & 17 & \\
\hline $\mathrm{C}_{2} \mathrm{H}_{3}$ & $\mathrm{H}$ & $\mathrm{C}_{2} \mathrm{H}_{2}$ & $\mathrm{H}_{2}$ & & $7.00 \mathrm{E}-12$ & 0.0 & 0.0 & 17 & \\
\hline$c$ & OH & $\mathbf{H}$ & $\mathrm{CO}$ & & $4.00 \mathrm{E}-11$ & 0.0 & 0.0 & $13, a$ & \\
\hline 0 & $\mathrm{CH}_{3}$ & $\mathrm{H}_{2} \mathrm{CO}$ & $\mathrm{H}$ & & $9.00 \mathrm{E}-11$ & 0.0 & 0.0 & 46 & \\
\hline 0 & $\mathrm{CH}_{2}$ & $\mathrm{CH}$ & $\mathrm{OH}$ & & $8.00 \mathrm{E}-12$ & 0.0 & 0.0 & $49, a$ & \\
\hline 0 & $\mathrm{CH}_{2}$ & $\mathrm{HCO}$ & $\mathbf{H}$ & & $1.00 \mathrm{E}-11$ & 0.0 & 0.0 & $49, a$ & \\
\hline $\mathrm{CH}_{2}$ & $\mathbf{N}$ & $\mathrm{HCN}$ & $\mathbf{H}$ & & $4.00 \mathrm{E}-11$ & 0.0 & 0.0 & $49, a$ & \\
\hline 0 & HCO & co & OH & & $1.23 E-10$ & 0.0 & 0.0 & 30 & \\
\hline 0 & $\mathrm{HCO}$ & $\mathrm{CO}_{2}$ & H & & $8.79 E-11$ & 0.0 & 0.0 & 30 & \\
\hline $\mathrm{HCO}$ & $\mathbf{H}$ & $\mathrm{CO}$ & $\mathrm{H}_{2}$ & & $7.38 \mathrm{E}-10$ & 0.0 & 0.0 & 30 & \\
\hline 0 & $\mathrm{OH}$ & $\mathrm{H}$ & $\mathrm{O}_{2}$ & & $4.15 \mathrm{E}-11$ & 0.0 & 0.0 & 51 & \\
\hline $\mathbf{N}$ & NH & $\mathrm{N}_{2}$ & H & & $4.00 E-11$ & 0.0 & 0.0 & $13, a$ & \\
\hline NH & $\mathrm{C}$ & $\mathrm{CN}$ & $\mathbf{H}$ & & $4.00 \mathrm{E}-11$ & 0.0 & 0.0 & $13, a$ & \\
\hline N & $\mathrm{CH}$ & $\mathrm{CN}$ & $\mathbf{H}$ & & $4.00 \mathrm{E}-11$ & 0.0 & 0.0 & $13, a$ & \\
\hline NH & 0 & NO & $\mathbf{H}$ & & $4.00 \mathrm{E}-11$ & 0.0 & 0.0 & $13, a$ & \\
\hline $\mathrm{NH}_{2}$ & 0 & $\mathrm{OH}$ & NH & & $1.75 \mathrm{E}-12$ & 0.0 & 0.0 & 50,1 & \\
\hline
\end{tabular}


Table IV (cont)

\begin{tabular}{|c|c|c|c|c|c|c|c|c|c|}
\hline \multicolumn{3}{|c|}{ Reactants } & \multicolumn{2}{|c|}{ Products } & \multirow{2}{*}{$\frac{A}{1.75 E-12}$} & \multirow{2}{*}{$\frac{B}{0.0}$} & \multirow{2}{*}{$\frac{\mathrm{c}}{0.0}$} & \multirow{2}{*}{$\frac{\operatorname{Refs} \text {. and }}{50,1}$} & \multirow{2}{*}{ d Noter } \\
\hline $\mathrm{NH}_{2}$ & 0 & & HNO & H & & & & & \\
\hline No & $\mathbf{N}$ & & $\mathrm{N}_{2}$ & 0 & $2.66 E-11$ & 0.0 & 0.0 & 30 & \\
\hline c & $\mathrm{O}_{2}$ & & co & 0 & $3.30 \mathrm{E}-11$ & 0.0 & 0.0 & 29 & \\
\hline C & No & & $\mathrm{CN}$ & 0 & $7.30 \mathrm{E}-11$ & 0.0 & 0.0 & 29 & \\
\hline $\mathrm{N}$ & $\mathrm{OH}$ & & no & $\mathbf{H}$ & $5.81 \mathrm{E}-11$ & 0.0 & 0.0 & 51 & \\
\hline $\mathrm{OH}$ & $\mathrm{OH}$ & & 0 & $\mathrm{H}_{2} \mathrm{O}$ & $1.05 E-11$ & 0.0 & 550. & 30 & \\
\hline co & $\mathrm{OH}$ & & $\mathrm{CO}_{2}$ & H & $9.30 E-13$ & 0.0 & 540 & 11 & \\
\hline $\mathbf{C H}$ & 0 & & $\mathrm{co}$ & H & $4.00 \mathrm{E}-11$ & 0.0 & 0.0 & $13, a$ & \\
\hline $\mathrm{CH}$ & 0 & & $\mathrm{HCO}^{+}$ & e & $1.00 \mathrm{E}-11$ & 0.0 & 0.0 & $47, m$ & \\
\hline $\mathrm{CH}$ & c & & $\mathrm{c}_{2}$ & $\mathbf{H}$ & $4.00 E-11$ & 0.0 & 0.0 & $33, a$ & \\
\hline$c_{2}$ & 0 & & co & C & $3.00 \mathrm{E}-11$ & 0.0 & 0.0 & $33, a$ & \\
\hline $\mathrm{CN}$ & $\mathrm{O}_{2}$ & & NCO & 0 & $7.00 \mathrm{E}-12$ & 0.0 & 0.0 & 46 & \\
\hline $\mathrm{CH}$ & H & & C & $\mathrm{H}_{2}$ & $1.00 \mathrm{E}-14$ & 0.0 & 0.0 & $33, a$ & \\
\hline $\mathrm{N}$ & $\mathbf{N}$ & $\mathrm{CO}_{2}$ & $\mathrm{~N}_{2}$ & $\mathrm{CO}_{2}$ & $1.30 \mathrm{E}-33$ & 0.0 & -500 & 30 & \\
\hline $\mathrm{N}$ & 0 & $\mathrm{H}_{2} \mathrm{O}$ & no & $\mathrm{H}_{2} \mathrm{O}$ & $6.90 \mathrm{E}-32$ & -0.5 & 0.0 & 30 & \\
\hline $\mathrm{N}$ & 0 & $\mathrm{CO}_{2}$ & No & $\mathrm{CO}_{2}$ & $3.00 E-32$ & -0.5 & 0.0 & 30 & \\
\hline H & н & M & $\mathrm{H}_{2}$ & M & $8.00 E-3 ?$ & -0.6 & 0.0 & 17 & \\
\hline $\mathrm{CH}_{3}$ & $\mathbf{H}$ & м & $\mathrm{CH}_{4}$ & M & $8.50 E-29$ & 0.0 & 0.0 & 17 & \\
\hline $\mathrm{CH}_{3}$ & $\mathrm{CH}_{3}$ & M & $\mathrm{C}_{2} \mathrm{H}_{6}$ & M & $6.00 \mathrm{E}-29$ & 0.0 & 0.0 & 17 & \\
\hline $\mathrm{C}_{2} \mathrm{H}_{2}$ & H & M & $\mathrm{C}_{2} \mathrm{H}_{3}$ & $M$ & $2.50 E-30$ & 0.0 & 0.0 & 17 & \\
\hline 0 & H & & $\mathrm{OH}$ & & $1.14 \mathrm{E}-19$ & 0.807 & 0.0 & $36, a, n$ & \\
\hline C & 0 & & Co & & $1.00 E-16$ & 0.0 & 0.0 & 31,2 & \\
\hline c & c & & $c_{2}$ & & $1.00 \mathrm{E}-16$ & 0.0 & 0.0 & $31, a$ & \\
\hline c & н & & $\mathrm{CH}$ & & $8.00 E-19$ & -1.2 & 0.0 & $33, a$ & \\
\hline C & $\mathbf{N}$ & & CN & & 2.0OE-17 & 0.0 & 0.0 & 11 & \\
\hline$O(1 D)$ & & & 0 & & $6.80 \varepsilon-03$ & 0.0 & 0.0 & 11 & \\
\hline$O(1 D)$ & $\mathrm{H}_{2} \mathrm{O}$ & . & $\mathrm{OH}$ & $\mathrm{OH}$ & 2. $10 E-10$ & 0.0 & 0.0 & 11,26 & \\
\hline$O(1 D)$ & $\mathrm{CO}_{2}$ & & 0 & $\mathrm{CO}_{2}$ & $1.20 E-10$ & 0.0 & 0.0 & 11 & \\
\hline$o(1 D)$ & $\mathrm{NH}_{3}$ & & $\mathrm{NH}_{2}$ & $\mathrm{OH}$ & $3.40 \mathrm{E}-10$ & 0.0 & 0.0 & 11 & \\
\hline$O(1 D)$ & $\mathrm{CH}_{4}$ & & $\mathrm{CH}_{3}$ & $\mathbf{O H}$ & $1.20 \mathrm{E}-10$ & 0.0 & 0.0 & 11 & \\
\hline$O(1 D)$ & $\mathrm{CH}_{4}$ & & $\mathrm{H}_{2} \mathrm{CO}$ & $\mathrm{H}_{2}$ & $1.30 E-11$ & 0.0 & 0.0 & 11 & \\
\hline$O(1 D)$ & co & & 0 & Co & $5.50 E-10$ & 0.0 & 625 & 53 & \\
\hline$O(1 D)$ & $\mathrm{H}_{2}$ & & OH & н & $1.30 \mathrm{E}-10$ & 0.0 & 0.0 & 11 & \\
\hline $\operatorname{co}(3)$ & & & co & & $3.10 E+02$ & 0.0 & 0.0 & 55 & \\
\hline
\end{tabular}


Table IV (cont)

\begin{tabular}{|c|c|c|c|c|c|c|}
\hline Reactants & Produ & $\operatorname{cts}$ & $\underline{\mathbf{A}}$ & $\underline{B}$ & C & Refs. and Notes \\
\hline $\operatorname{co}(3) \mathrm{M}$ & $\mathrm{CO}$ & M & $2.00 \mathrm{E}-10$ & 0.0 & 0.0 & $\mathbf{a}$ \\
\hline$C(1 D)$ & $\mathrm{C}$ & & $3.10 E-04$ & 0.0 & 0.0 & 11 \\
\hline $\mathrm{C}$ (1D) $\mathrm{CH}_{4}$ & $\mathrm{C}_{2} \mathrm{H}_{2}$ & $\mathrm{H}_{2}$ & $3.20 \mathrm{E}-11$ & 0.0 & 0.0 & 54 \\
\hline$C(1 D) H_{2}$ & $\mathrm{CH}$ & $\mathbf{H}$ & $4.15 E-11$ & 0.0 & 0.0 & 54 \\
\hline$C(1 D)$ NO & $\mathrm{CN}$ & 0 & $9.20 \mathrm{E}-11$ & 0.0 & 0.0 & 54 \\
\hline$C(1 D) M$ & $c$ & $M$ & $2.00 \mathrm{E}-10$ & 0.0 & 0.0 & a \\
\hline
\end{tabular}

a Estimate.

b From extrapolation to low temperature of Bates's Table 2 (Ref. 35).

c From detafled balance of reverse reaction.

d $\sim 10^{-10} \mathrm{~cm}^{3} \mathrm{~s}^{-1}$ at $100 \mathrm{~K}$ is considered an upper limit by rrauss and Julienne (Ref. 20).

e A11 branches observed with $\mathrm{HCO}^{+}$being the major product ion; branching ratios are our estimate.

f Preliminary result.

$g$ These branches in addition to branches proposed by Herbst and Klemperer (Ref. 13), which doubles the overall rate.

$\mathrm{h}$ Assumes. Herbst and Klemperer (Ref. 13) rate was estimated for $30 \mathrm{~K} ; \mathrm{T}^{-0.5}$ variation.

1 Effect of three-body association removed.

j Huntress (Ref. 39) gives stmilar preliminary result in which the rate coefficient is $9.6 \times 10^{-10} \mathrm{~cm}^{3} \mathrm{~s}^{-1}$.

$k$ Our estimate for temperature dependence.

1 Branching ratios are our estimate.

m Calculated; perhaps a lower $1 \mathrm{imit}$, especially for our coma temperatures.

n From fit, $T=200-300 \mathrm{~K}$.

C. Combining Physics and Chemistry

In the computer model the physical and chemical processes are analyzed in a thin spherical shell of coma gas as it expands and moves radially outward. Chemical reactions take place in a continually diluting gas exposed to a continually less attenuated solar ultraviolet flux. Because of the motion and progressive dilution of the gas, many reactions will not reach steady-state conditions so that the abundances of some species, that otherwise might have undergone further reactions, will "freeze-in." 


\section{PARAMETERS}

The size of the nucleus, its albedo, and infrared emissivity are not known for $\mathrm{P} / \mathrm{Halley}$ and most other comets. We have adopted a standard set of values in agreement with other investigators (Ref. 2). For this standard nucleus the mean radius is $R_{0}=2.5 \mathrm{~km}$, its visual albedo $A=0.3$, and 1 ts infrared emissivity is assumed to be $\varepsilon=1-A=0.7$. Extreme values for the nuclear mean radius are thought to be $0.5 \mathrm{~km}$ and $5.0 \mathrm{~km}$. Extremes for the visual albedo are assumed to be $A=0.1$ and $A=0.7$. The corresponding values for the infrared enissivity are assumed to be $\varepsilon=1-A$. Gas production rates, $Z$, consistent with chemical composition $6 a$ are given in Table V. For this composition and the standard nucleus the total gas production rate at $r=1$ AU is $Q=4 \pi R_{0}^{2} \mathrm{Z}=2.8 \times 10^{29}$ molecules $\mathrm{s}^{-1}$. This is about $2 / 3$ more than the predicted nominal gas production of $1.7 \times 10^{29} \mathrm{~s}^{-1}$ (see Ref. 2).

Table V

Gas Production Rates, $\mathrm{Z}\left(10^{17}\right.$ Molecules $\left.\mathrm{cm}^{-2} \mathrm{~s}^{-1}\right)$

\begin{tabular}{lcccc} 
& \multicolumn{3}{c}{$\mathrm{A}$} \\
\cline { 2 - 5 } $\mathbf{n}$ & 0.1 & 0.3 & 0.5 & 0.7 \\
\hline & & & & \\
0.6 & 13.5 & 10.5 & 7.53 & 4.53 \\
1.0 & 4.60 & 3.59 & 2.58 & 1.56 \\
1.5 & & 1.47 & & \\
2.0 & & 0.715 & & 0.0997 \\
3.0 & 0.262 & 0.211 & 0.157 & \\
5.0 & & 0.00843 & &
\end{tabular}


In trying to assimflate a consistent picture of comet Halley from the model presented here, establishment of some type of systematic criterion is required for comparison with the 1910 observations. It is difficult at best to make any direct correlations between Intensities of spectral images and colum densities. An attempt to compare observational plates from the 1910 apparition (and inferences made thereof) to the computer model's column density predictions would be particularly difficult because of the suspect quality of the 1910 observations as discussed in Sec. I. However, to facilitate understanding and thus determine the correct behavior of the model in relation to the 1910 apparition, a lower cutoff in column derisity of about $10^{11} \mathrm{cN}$ or $\mathrm{C}_{2}$ molecules $\mathrm{cm}^{-2}$ is somewhat arbitrarily imposed for the purpose of comparing with the observed intensities. It is felt that this limit is entirely reasonable in view of observations of more recent comets. Simflar arguments hold for $\mathrm{C}_{3}$ for the unmodifled composition $6 a$ (as given in Table II), assuming the incorrect oscillator strength that is too low by a factor 40 . For the modified composition 6a' (assuming the correct oscillator strength), the cutoff 1 imit for $\mathrm{C}_{3}$ has to be lowered by a factor of about 40. A further, but somewhat arbitrary, criterion is that when not dealing explicitly with envelope extent as delineated by the coma projection distance $\rho$, all column density values are given as averaged out to $\rho=10^{4} \mathrm{~km}$. That this approach is meaningful can be seen from the fact that most of the physics and chemistry occurs within this distance, thereby giving credence to this value of $\rho$ and the averaged column densities.

To discuss the results in a logically ordered manner, attention will be focused on the "standard" model of comet Halley; 1.e. -- a 2.5-km nuclear radius and visual albedo $A=0.3$, and $\mathrm{z}$ of $\sim 10^{17}-10^{18}$ molecules $\mathrm{cm}^{-2} \mathrm{~s}^{-1}$ at $1 \mathrm{AU}$. The extremes considered in this study can then be viewed in relation to this standard, allowing for a better picture of $\mathrm{P} / \mathrm{Halley}$ and thus refinements in the model. As an ald in comparing the model's results to the 1910 observational sketch as given in Table I, a similar, though discussion orientated, trace will be made here.

At a comparable hellocentric distance of $3.0 \mathrm{AU}$ (F1g.1) the standard nucleus exhibits a faint, small halo $\left(\sim 4\right.$ to $\left.5 \times 10^{3} \mathrm{~km}\right)$ of $\mathrm{CN}$. Though below the Imposed lower cutoff in colum density, $C_{3}$ is such that a faint observation is 
possible. $C_{2}$ on the other hand, being an order of magnitude lower than $C_{3}$, should not be visible, supporting the argument ${ }^{2}$ that $C_{2}$ was only doubtfully seen at this distance.

Coming to 2.0 AJ (Fig.2) one finds $\mathrm{CN}$ and $\mathrm{C}_{3}$ vistble with envelope extensions of $\sim 1$ to $2 \times 10^{5} \mathrm{~km}$ and $\sim 2$ to $3 \times 10^{4} \mathrm{~km}$, respective1y. $\mathrm{C}_{2} 1 \mathrm{~s}$ sti11 below cutoff by roughly a factor of 4 .

By $1.0 \mathrm{AU}(\mathrm{Fig} .2) \mathrm{C}_{2}$ becomes visible with a coma dimension of $\sim 2 \times 10^{5} \mathrm{~km}$. For $C N$ and $C_{3}$ we have envelope slzes of $\sim 4$ and $2 \times 10^{5} \mathrm{~km}$, respectively. In the inner coma out to approximately $6 \times 10^{4} \mathrm{~km}, C_{3}$ has clear dominance over $C_{2}$, thereafter $\mathrm{C}_{2}$ becomes dominant and approaches (within a factor of 2 to 3 ) $\mathrm{CN}$ abundaces in the far outer regions. One can easily notice (FIgs.3 and 4) that for larger nuclear radil this crossing distance $\left(c_{3}\right.$ to $c_{2}$ dominance) diminishes, the reverse being true for smaller radil. $C_{2}$ production has thus increased in relation to $\mathrm{CN}, \mathrm{C}_{3}$, and $\mathrm{OH}$ as can be seen in Fig.5. Up to this point, $\mathrm{CN}_{3} \mathrm{C}_{3}$, and $\mathrm{OH}$ have exhibited similar behavior, and readily seen is the steepening slope of $C_{2}$ In relation to these specles; $C_{2}$ is on a faster $r$ ise, giving some credence to the argument ${ }^{1}$ that an actual spectral change occurred around 1 to $1.2 \mathrm{AU}$. Fig.6 exhibits this rise in $\mathrm{C}_{2}$ for the extremes in nuclear radil. Note the slightly more acute slope for the larger nuclear radil on approach to the sun. $\mathrm{C}_{2}$ production is thus apparently favored by larger nuclear sizes. Referring to the sequential plots (FIgs.7 through 10) shows this effect quite nicely, with $\mathrm{OH} / \mathrm{CN}, \mathrm{OH} / \mathrm{C}_{3}$, and $\mathrm{CN} / \mathrm{C}_{3}$ ratios remaining roughly constant and $\mathrm{C}_{2}$ rising (gharper for larger $R_{0}$ ) in relation.

Perthelion being at approximately 0.6 AU for P/Halley (Fig.1) shows the standard model exhibiting strong $\mathrm{CN}$ and $\mathrm{C}_{2}$ (comparable) in the outer coma (envelope dimensions both $\leq 5 \times 10^{5} \mathrm{~km}$ ). The $\mathrm{C}_{3}-\mathrm{C}_{2}$ dominance crossing distance has decreased to $\sim 10^{4} \mathrm{~km}$ with the $C_{3}$ halo having an $\sim 10^{5}-\mathrm{km}$ size.

Thus far discussion has been made concerning only the species $C_{1}, C_{2}, C_{3}$, and $\mathrm{OH}$, but what of the ionic behavior? of particular interest, two lonic species, $\mathrm{H}_{2} \mathrm{O}^{+}$and $\mathrm{CO}^{+}$should be noted. $\mathrm{FIg.11}$ shows these species' varlations with heliocentric distance for three nuclear dimensions; $5.0 \mathrm{~km}, 2.5 \mathrm{~km}$, and $0.5 \mathrm{~km}$. Note the changes in slope and dominance with respect to nuclear size. In relation to similar plots ( $F 1 g s .5$ and 6 ) for the neutrals the lonic behavior is noticeably different. 
The standard model in these lons is shown in Fig.12 for these hellocentric distances. Again, with approach to the Sun, the dominance crossing distance decreases (a limit being reached at $\sim 1.0 \mathrm{AU}$ ), $\mathrm{CO}^{+}$the stronger in the outer regions. A rather strange effect can easily be seen in this plot; with solar approach $\mathrm{CO}^{+}$and $\mathrm{H}_{2} \mathrm{O}^{+}$are significantly depleted in the far coma yet increasingly abundant in the inner regions. This could possibly be explained if solar wind loading and lonlc sweep out were taken into account, however, in this present model they are not. Evidently, this then 1 s an ini insic effect, explainable on physio-chemical grounds.

At a given helfocentric distance (such as $1.0 \mathrm{AU}$ in Fig.13) one sees roughly the same behavior in these lons over the ranges of nuclear sizes considered, the only difference being the crossing distance of dominance shift in $\mathrm{H}_{2} \mathrm{O}^{+}$and $\mathrm{CO}^{+}$; larger nuclear radif yielding increasing crossing distance (the opposite effect of the neutrals considered).

Figs.14-18 give information cn variation with albedo for the neutrals mentioned above. One can see that similar behavior is exhibited by all of these species. No significant variation is apparent with respect to either nuclear size or helfocentric distance. At a given heliocentric distance and for a particular nuclear radius a variation in albedo of 0.1 to $0.7 \mathrm{simply}$ decreases the abundance by roughly a factor of 3 (about the same for all species shown).

The first 18 figures are based on composition $6 \mathrm{a}$. Fig.19 is for composition $6 \mathrm{a}^{\circ}$. Since the relative abundances of $\mathrm{C}_{2} \mathrm{H}_{2}$ and $\mathrm{H}_{2} \mathrm{C}_{3} \mathrm{H}_{2}$ are very small, the changes in these abundances affect only the production of $c_{3}$; the other observable species remain essentially unchanged. The $C_{3}$ curves could thus be scaled down by a factor of 40 in Figs. 1-10 and 14-18 without affecting the other curves. This is borne out by the results of test calculations presented in Fig.19 that can be compared with FIg.5.

\section{v. CONCLUSIONS}

The model with the standard size nucleus, $R_{0}=2.5 \mathrm{~km}$, compares favorably with the observations from 1910. The smallest nucleus, $R_{0}=0.5 \mathrm{~km}$, compares least favorably, giving column densities which are too low even for the relatively volatile composition assumed here. Results from the model are too insensitive to indicate a preference for albedo and emissivity. The column 
density profiles are nearly flat, increasing only very slowly with decreasing projected distance from the nucleus. Thus, a spacecraft approaching the nucleus from a distance 1 ess than a few $10^{4} \mathrm{~km}$ will "see" only very small changes in the intensity of $\mathrm{C}_{2}, \mathrm{C}_{3}, \mathrm{CN}, \mathrm{OH}$, and most other molecular radicals.

\section{ACKNOWDLEDAMENTS}

We are indebted to $P$. T. Giguere for his dedication in checking reaction rates and references and to J. J. Keady for helpful discussions. 


\section{REFERENCES}

1. N. T. Bobrovnikoff, "On the Spectrum of Halley's Comet," Astrophys. J. $\underline{66}$, 145-169 (1927).

2. R. L. Newburn, Jr., and D. K. Yeomans, "Halley's Comet," Jet Propulsion Laboratory report 900-999 (July 1981). Also condensed version: Ann. Rev. Earth Planetary Sclences, to be published (1982).

3. N. T. Bobrovnikoff, "Physical Theory of Comets in the Light of Spectroscopic Data," Rev. Mod. Phys. 14, 164-178 (1942).

4. R. von Mises, Mathematical Theory of Compressible Fluid Flow (Academic Press, New York, 1958), pp. 73-77.

5. W. F. Huebner and P. T. Giguere, "A Model of Comet Comae. II. Effects of Solar Photodissociative Ionization," Astrophys. J. 238, 753-762 (1980).

6. W. F. Huebner, "Chemical Kinetics in the Coma," in Comets and the Origin of Life, C. Ponnamperuma, Ed. (D. Reldel Publishing Company, Dorderecht, Ho11and, 1981), pp. 91-103.

7. W. F. Huebner, P. T. Giguere, and W. L. Slattery, "Photochemical Processes In the Inner Coma," in Comets, L. L. Wilkening, Ed. (University of Arizona Press, 1982), pp. 496-515.

8. L. Blermann, P. T. Giguere, and W. F. Huebner, "A Model of a Comet Coma with Interstellar Molecules in the Nucleus," Astron. Astrophys. In press (1982).

9. Michae1 F. A'Hearn, Un1v. of Maryland, letter to Huebner (September 1981 ).

10. W. T. Huntress, Jr., "Laboratory Studies of Bimolecular Reactions of Positive Ions in Interstellar Clouds, in Comets, and in Planetary Atmospheres of Reducing Composition," Astrophys. J. Suppl. 33, 495-514 (1977).

11. T. Baurer and M. H. Bortner, "Summary of Suggested Rate Constants," in Defense Nuclear Agency Reaction Rate Handbook, M. H. Bortner, T. Baurer,

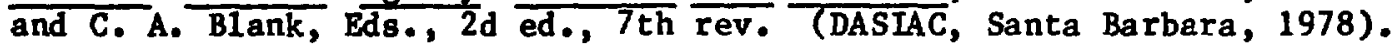

12. E. E. Ferguson, "Rate Constants of Thermal Energy Binary Ion-Molecule Reactions of Aeronomic Interest," Atomic Data Nucl. Data Tables 12, 159-178 (1973).

13. E. Herbst and W. Klemperer, "The Formation and Depletion of Molecules in Dense Interste1lar Clouds," Astrophys. J. 185, 505-533 (1973).

14. M. Oppenheimer, "Gas Phase Chemistry in Comets," Astrophys. J. 196, 251-259 (1975).

15. Z. Karpas and W. T. Huntress, Jr., "Reactions of $\mathrm{OH}^{+}$and $\mathrm{H}_{2} \mathrm{O}^{+}$Ions with Some Diatomic and Simple Polyatomic Molecules," Chem. Phys. Letters 59, 87-89 (1978). 
16. F. C. Fehsenfeld, "Ion Reactions wIth Atomic 0xygen and Atomic Nitrogen of Astrophysical Importance," Astrophys. J. 209, 638-639 (1976).

17. L. A. Capone, R. C. Whitten, J. Dubach, S. S. Prasad, and W. T. Huntress, Jr., "The Lower Ionosphere of Titan," Icarus 28, 367-378 (1976).

18. R. Johnsen and M. A. Biond1, "Measurements of Positive Ion Conversion and Removal Reactions Relating to the Jovian Ionosphere," Icarus 23, 139-143 (1974).

19. E. Herbst, J. G. Schubert, and P. R. Certain, "The Radiative Association of $\mathrm{CH}_{2}{ }^{+}$," Astrophys. J. 213, 696-704 (1977).

20. M. Krauss and P. S. Julienne, "Dissociative Recombination of e $+\mathrm{CH}^{+}(\mathrm{X}$ $\left.1_{\Sigma}^{+}\right), "$ Astrophys. J. (Letters) 183, L139-L141 (1973).

21. R. E. Rebbert, S. G. Llas, and P. Ausloos, "Pulse Radiolysis of Methane," J. Res. NBS 77A, 249-257 (1973).

22. F. C. Fehsenfeld, W. Lindinger, A. L. Schmeltekopf, D. L. Albriţton, and E. E. Ferguson, "Energy Dependence of the Reaction $\mathrm{NH}_{3}^{+}+\mathrm{H}_{2}+\mathrm{NH}_{4}^{+}+\mathrm{H}$," J. Chem. Phys. 62, 2001-2003 (1975).

23. R. E. Rebbert and P. Ausloos, "Radiolysis of Methane. Formation of Acetylene," J. Res. NBS 77A, 109-114 (1973).

24. E. Herbst, D. K. Bohme, J. D. Payzant, and H. I. Schiff, "Rate of the Reaction $\mathrm{N}_{2} \mathrm{H}^{+}+\mathrm{CO}+\mathrm{HCO}^{+}+\mathrm{N}_{2}$ and Its Significance for the Interstellar Chemistry of $\mathrm{N}_{2} \mathrm{H}^{+}$," Astrophys. J. 201, 603-606 (1975).

25. W. F. Huebner and C. W. Carpenter, "Solar Photo Rate Coefficlents," Los Alamos Scientiflc Laboratory report LA-8085-MS (1979).

26. R. F. Heidner and D. Husain, "Electrontcally Excited Oxygen Atons, $O\left(2^{l} D_{2}\right)$. A Time-Resolved Study of the Collisional Quenching by the Gases $\mathrm{H}_{2}, \mathrm{D}_{2}$, $\mathrm{No}$, $\mathrm{N}_{2} \mathrm{O}, \mathrm{CH}_{4}$, and $\mathrm{C}_{3} \mathrm{O}_{2}$ Using Atomic Absorption Spectroscopy in the Vacuum Ultraviolet," Int. J. Chem. Kinet. 5, 819-831 (1973).

27. W. Braun, A. M. Bass, and M. P1lling, "Flash Photolysis of Ketene and Diazomethane: The Production and Reaction Kinetics of Triplet and Singlet Methylene," J. Chem. Phys. 52, 5131-5143 (1970).

28. Slanger, T. G., "Studies of Vibrationally Excited $\mathrm{H}_{2}, "$ 3rd Annual NASA Planetary Atmospheres Principal Investigators Conf., Greenbelt, ${ }^{.}$, , Feb. 9-12, 1981, Pp. XIV-6--XIV-7.

29. D. Husain and L. J. Kirsch, "Reactions of Atonic Carbon $C\left(2{ }^{3} P_{J}\right)$ by KInetic Absorption Spectroscopy in the Vacuum U1tra-Violet," Trans. Faraday Soc. 67, 2025-2035 (1971).

30. D. L. Baulch, D. D. Drysdale, D. G. Horne, and A. C. Lloyd, Evaluated Kinetic Data for High Temperature Reactions, (Chemical Rubber Co. Press, Cleveland, $1 9 7 2 \longdiv { \text { . } }$ 
31. W. D. Watson, "Physical Processes for the Formation and Destruction of Interstellar Molecules," in Atomic and Molecular Physics and the Interstellar Matter, R. Balian, P. Encrenaz, and J. Lequeux, Eds. (North Holland, Amsterdan, 1975), pp. 177-324.

32. M. T. Leu, M. A. Biond1, and R. Johnsen, "Measurements of Recombination of Electrons with HCO ${ }^{+}$Ions," Phys. Rev. A8, 420-422 (1973).

33. W. Klemperer, "Interstellar Molecile Formation, Radiative and Exchange Reactions," in Highlights of Astronomy, Vol. 2, C. de Jager, Ed. (D. Reidel Publishing Co., Dordrecht, Holland, 1971), pp. 421-428.

34. J. N. Bardsley and B. R. Junker, "Di ssoclative Recombination of $\mathrm{CH}^{+}$Ions," Astrophys . J. (Letters) 183, L135-L137 (1973).

35. D. R. Bates, "Rate of Formation of Molecules by Radiative Association," MNRAS 111, 303-314 (1951).

36. P. S. Julienne and M. Krauss, "Molecule Formation by Inverse Predissociation," In Molecules in the Galactic Environment, M. A. Gordon and L. E. Snyder, Eds. (John wiley and Sons, New York, 1973), pp. 354-373.

37. Z. Karpas, V. G. AnIcIch, and W. T. Huntress, Jr., "An Ion Cyclotron Resonance Study of Reactions of Ions with Hydrogen Atoms," J. Chem. Phys. 70, 2877-2881 (1979).

38. D. Soith and N. G. Adams, "Molecular Synthesis in Interstellar Clouds: Some Relevant Laboratory Measurements," Astrophys. J. 217, 741-748 (1977).

39. W. T. Huntress, Jr., Jet Propulsion Laboratory, Pasadena, Cal., memo to P. T. Giguere (1978).

40. W. T. Huntress, Jr., and V. G. Anicich, "Laboratory Studies of Ion-Neutral Reactions in Interstellar Regions: Gas-Phase Equilibrium Between HCN and $\mathrm{NH}_{3}$ In Dense Clouds," Astrophys. J. 208, 237-244 (1976).

41. D. Smith, N. G. Adems, and $T_{f}$ M. Miller, "A Laboratory Study of the Reactions of $\mathrm{N}^{+}, \mathrm{N}_{2}^{+}, \mathrm{N}_{3}^{+}, \mathrm{N}_{4}^{+}, \mathrm{O}^{+}, \mathrm{O}_{2}^{+}$, and $\mathrm{NO}^{+}$Ions with Several Molecules at $300 \mathrm{~K}, " \mathrm{~J}$. Chem. Phys. 69, 308-318 (1978).

42. D. Smith and N. G. Adams, "Reaction of Simple Hydrocarbon Ions with Molecules at Thermal Energtes," Int. J. Mass Spectr. Ion Phys. 23, 123-135 (1977).

43. W. T. Huntress, Jet Propulsion Laboratory, Pasadena, Cal., letter to Huebner (1978).

44. Z. Karpas, V. G. Antcich, and W. T. Huntress, Jr., "An Ion Cyclotron Resonance Study of Reactions of Some Atomlc and Simple Polyatomic Ions with Water," Chen. Phys. Letter 59, 84-86 (1978).

45. F. C. Fehsenfeld, A. L. Schmeltekopf, and E. E. Ferguson, "Thermal Energy Ion-Neutral Reaction Rates. V. Measured Rate Constants for $\mathrm{C}^{+}$and $\mathrm{CO}^{{ }^{7}}$ Reactions with $\mathrm{O}_{2}$ and $\mathrm{CO}_{2}$," J. Chem. Phys. 45, 23-25 (1966). 
46. E. Igles1as, "The Chemical Evolution of Molecular Clouds," Astrophys. J. 218, 697-715 (1977).

47. M. MacGregor and R. S. Berry, "Formation of $\mathrm{HCO}^{+}$by the Associative Iontzation of $\mathrm{CH}+0$," J. Phys. B6, 181-196 (1973).

48. D. F. Strobel, "The Photochemistry of Hydrocarbons, in the Jovian Atmos phere," J. Atmos. Sc1. 30, 489-498 (1973).

49. J. H. Black, Diffuse Interstellar Clouds, Ph.D. Thesis, Harvard University (Apri1, 1975).

50. M. Gehring, K. Hoyermann, H. Schacke, and J. Wolfrum, "Direct Studies of Some Elementary Steps for the Formation and Destruction of Nitric Oxide in the H-N-O System," in Fourteenth Symposium (International) on Combustion (P1ttsburgh, Combustion Institute, 1973), pp. 99-105.

51. W. E. Wilson, Jr., "A Critical Review of the Gas-Phase Reaction Kinetics of the Hydroxyl Radical," J. Phys. Chem. Ref. Data 1, 535-573 (1972).

52. J. W. McGowan, P. M. Mul, V. S. D'Angelo, J. B. A. Mitchell, P. Defrance, and H. R. Froelich, "Energy Dependence of Dissociative Recombination Below $0.08 \mathrm{eV}$ Measured with (Electron-Ion) Merged-Beam Technique," 'Sys. Rev. Letters 42, 373-375 (1979) and 42, 1186 (1979).

53. R. Simonaitis and J. Heicklen, "The Reaction of $\mathrm{OH}$ with $\mathrm{NO}_{2}$ and the Deactivation of $O\left({ }^{1} \mathrm{D}\right)$ by CO," Int. J. Chem. Kin. 4, 529-540 (1972).

54. W. Braun, A. M. Bass, D. D. Davis, and J. D. Simmons, "Flash Photolysis of Carbon Suboxide: Absolute Rate Constants for Reactions of $C\left({ }^{3} \mathrm{P}\right)$ and $\mathrm{C}\left({ }^{1} \mathrm{D}\right)$ with $\mathrm{H}_{2}, \mathrm{~N}_{2}, \mathrm{CO}, \mathrm{NO}, \mathrm{O}_{2}$, and $\mathrm{CH}_{4}, "$ Proc. Roy. Soc. A312, 417-434 (1969).

55. L. A. Kuznetsova, N. E. Kuz'menko, Yu. Ya. Kuzyakov, and Yu. A. Plestinin, "Probabilities of Optical Transitions in Electronic Vibration-Rotation Spectra of Diatomic Molecules," Sov. Phys. Usp. 17, 405-423 (1974). 


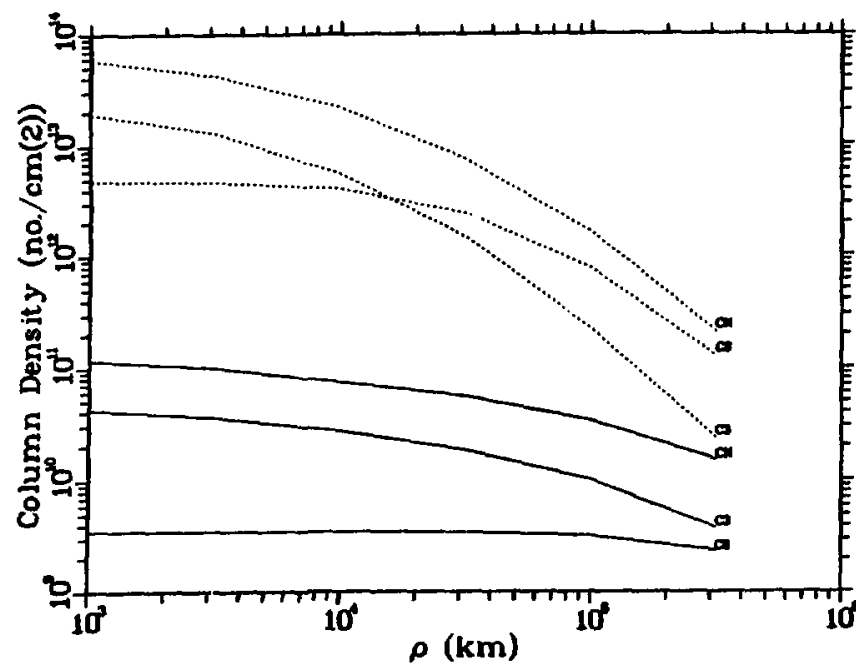

\section{Fig.1}

Column density vs. projected distance in coma for the standard model $(2.5 \mathrm{~km}$ nuclear radius, $A=0.3)$ in $\mathrm{CN}, \mathrm{C}_{2}$, and $\mathrm{C}_{3}$ at two heliocentric distances: solid line $r=3.0 \mathrm{AU}$, dashed line $r=0.6 \mathrm{AU}$.

F18.2

Column density v8. projected distance in coma for the standard mode1 $(2.5 \mathrm{~km}$ nuclear radius, $A=0.3)$ in $\mathrm{CN}, C_{2}$, and $C_{3}$ at two heliocentric distances: solid line $r=2.0 \mathrm{AU}$, dashed line $r=1.0 \mathrm{AU}$.
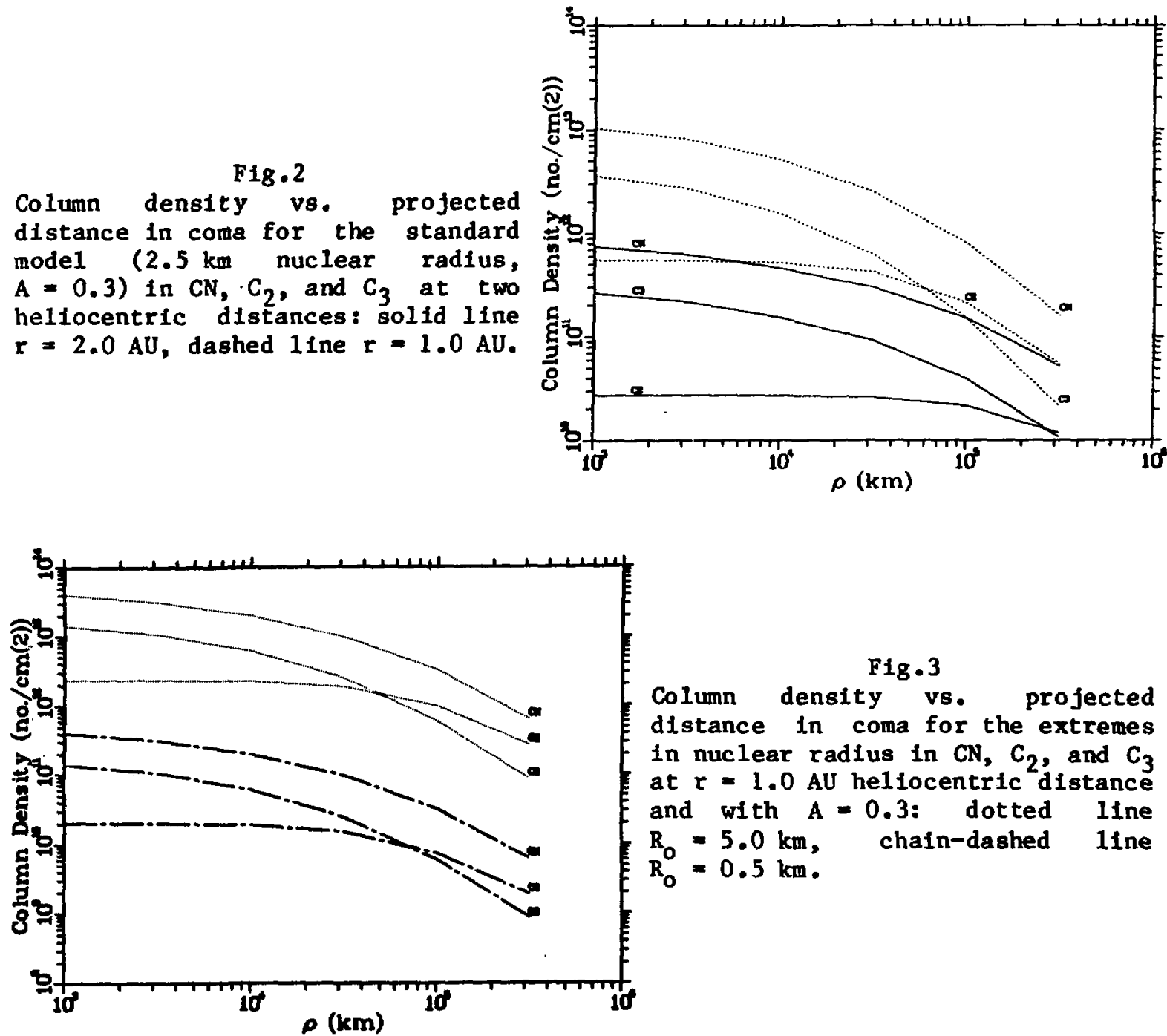

F1g.3

Column density vs. projected distance in coma for the extremes in nuclear radius in $C N, C_{2}$, and $C_{3}$ at $r=1.0 \mathrm{AU}$ heliocentric distance and with $A=0.3:$ dotted ilne $R_{0}=5.0 \mathrm{~km}$, chain-dashed line $R_{0}=0.5 \mathrm{~km}$. 


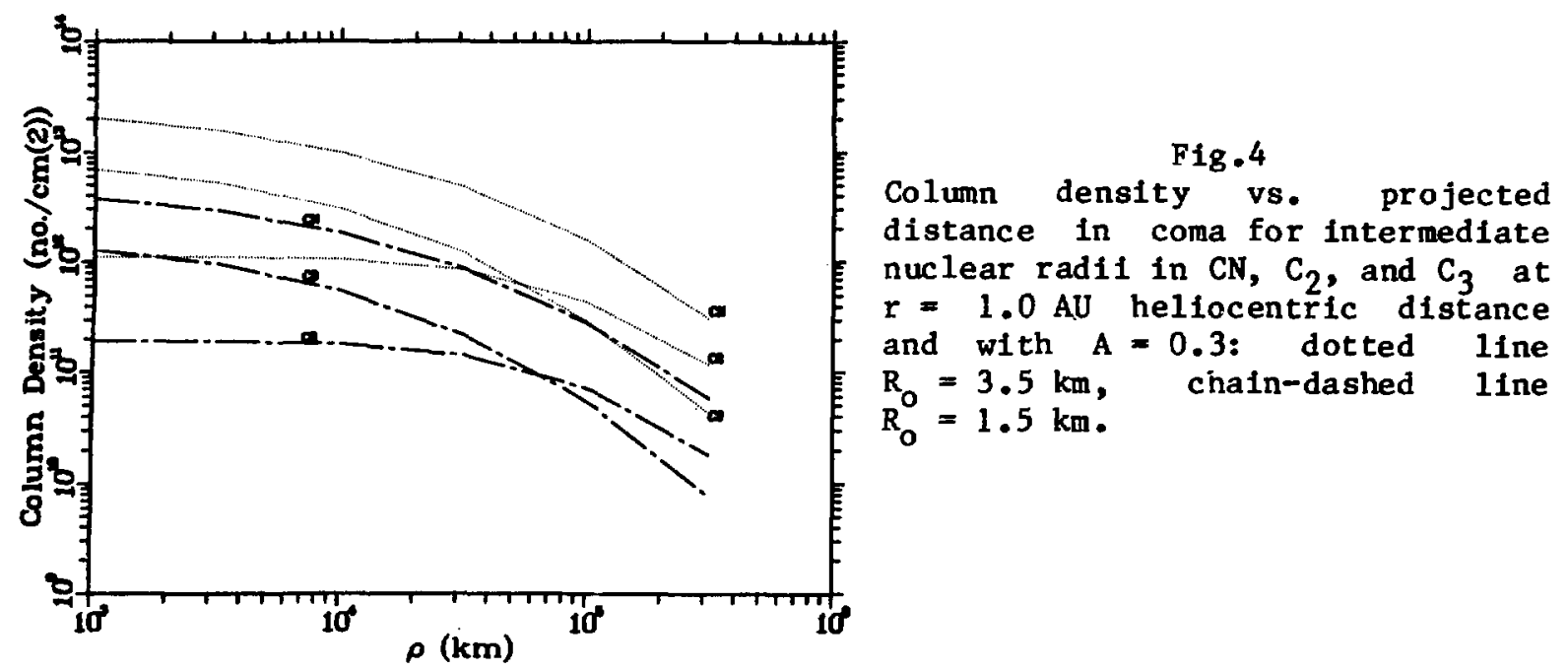

Fig.5

Column densities averaged to $\rho=10^{4} \mathrm{~km}$ of $\mathrm{CN}, \mathrm{C}_{2}, \mathrm{C}_{3}$, and $\mathrm{OH}$ as a function of heliocentric distance for the standard model. Note the steepening slope of $\mathrm{C}_{2}$ relative to the other constituents at small heliocentric distances.
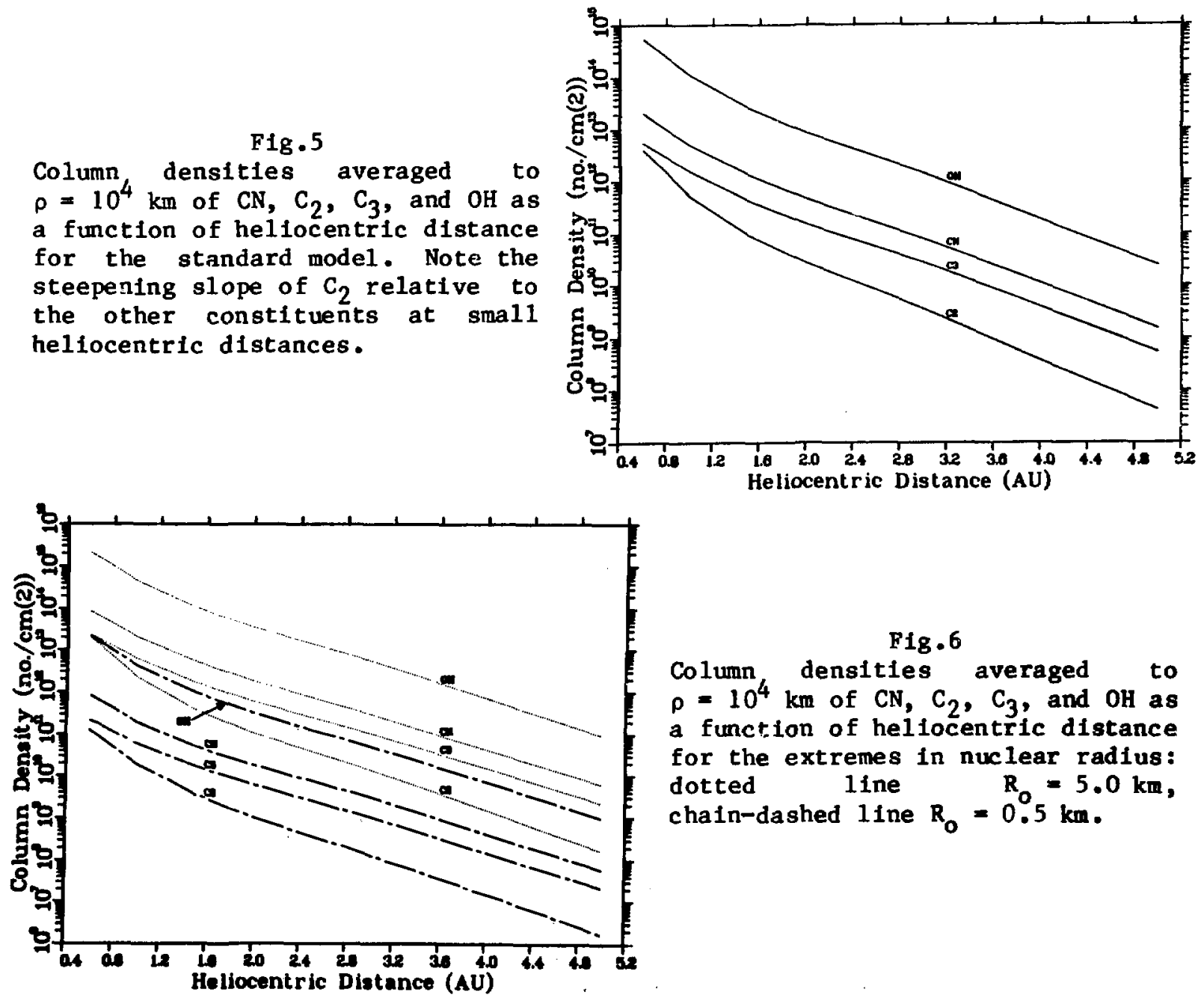

Fig.6

Column densities averaged to $\rho=10^{4} \mathrm{~km}$ of $\mathrm{CN}, \mathrm{C}_{2}, \mathrm{C}_{3}$, and $\mathrm{OH}$ as a function of hellocentric distance for the extremes in nuclear radius: dotted line $R_{0}=5.0 \mathrm{~km}$, cha1n-dashed 1 ine $R_{0}=0.5 \mathrm{~km}$. 


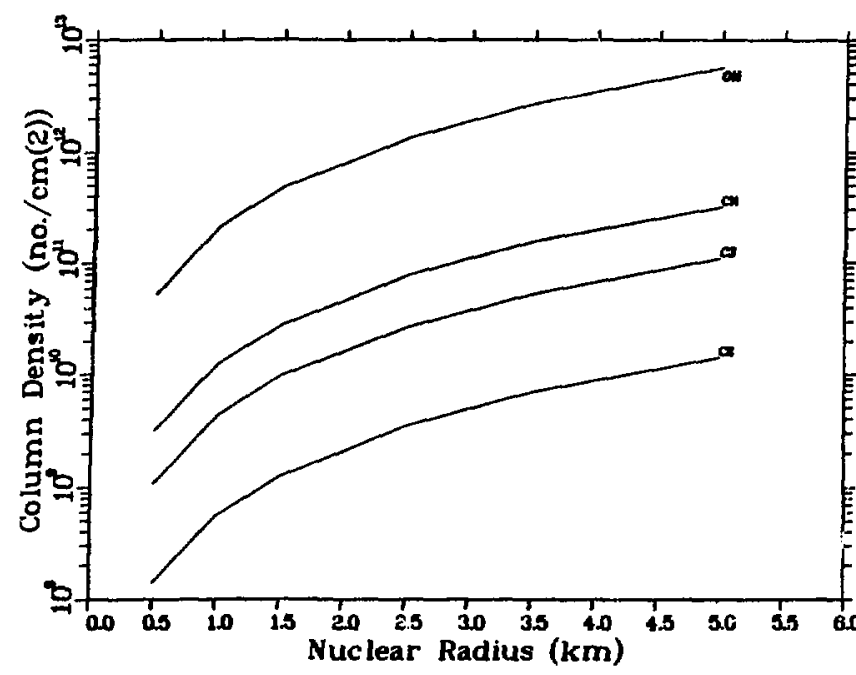

Fig.7

Column densities averaged to $\rho=$ $10^{4} \mathrm{~km}$ of $\mathrm{CN}, \mathrm{C}_{2}, \mathrm{C}_{3}$, and $\mathrm{OH}$ as a function of nuclear radius with $A=0.3$ at a helfocentric distance $r=3.0 \mathrm{AU}$.

Fig.8

Column densities averaged to $\rho=10^{4} \mathrm{~km}$ of $\mathrm{CN}, \mathrm{C}_{2}, \mathrm{C}_{3}$, and $\mathrm{OH}$ as a function of nuclear radius with $A=0.3$ at a hellocentric distance $r=2,0 \mathrm{AU}$.
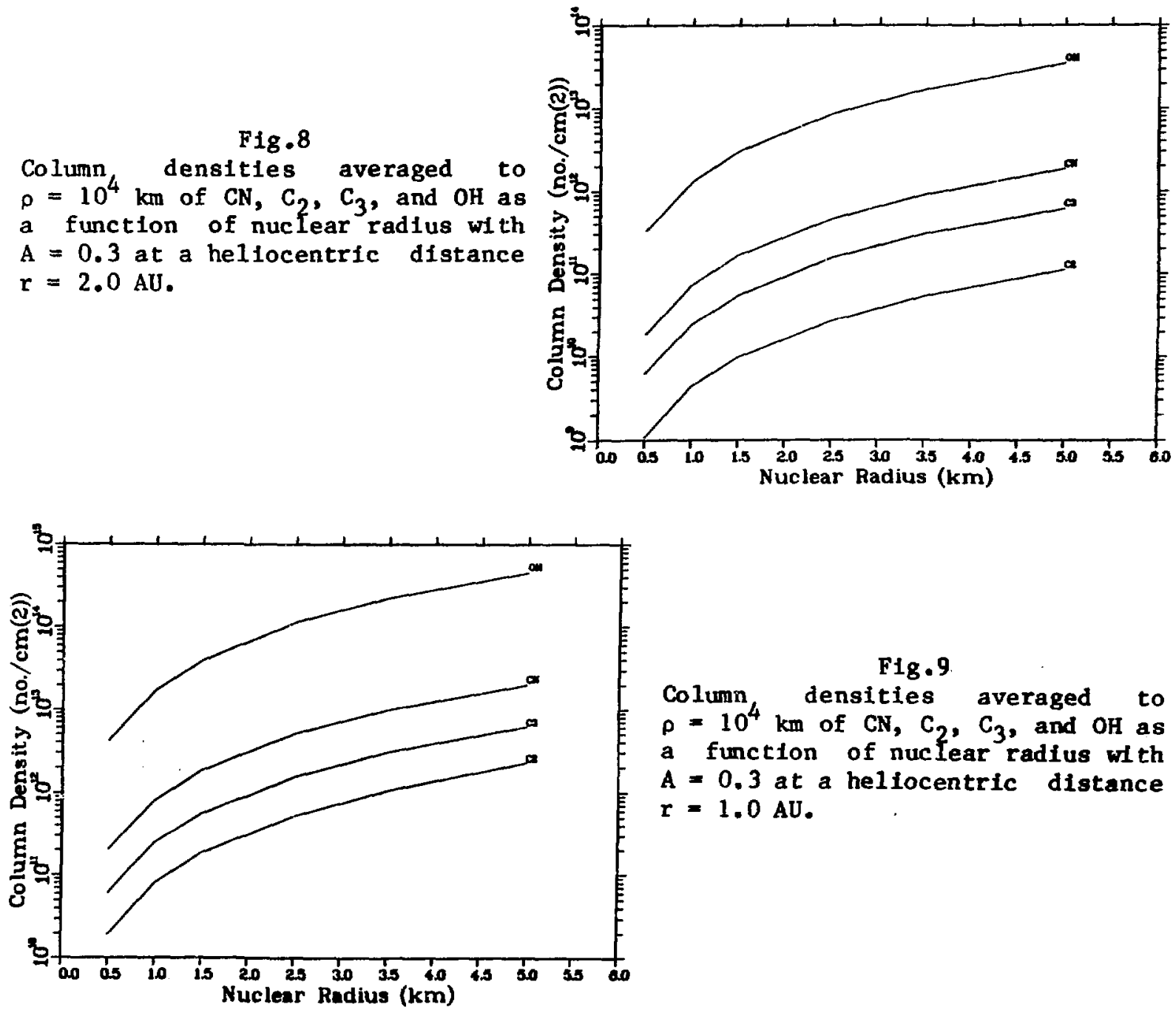

Fig.9

Column densities averaged to $\rho=10^{4} \mathrm{~km}$ of $\mathrm{CN}, \mathrm{C}_{2}, \mathrm{C}_{3}$, and $\mathrm{OH}$ as a function of nuclear radius with $A=0.3$ at a heliocentric distance $r=1.0 \mathrm{AU}$. 

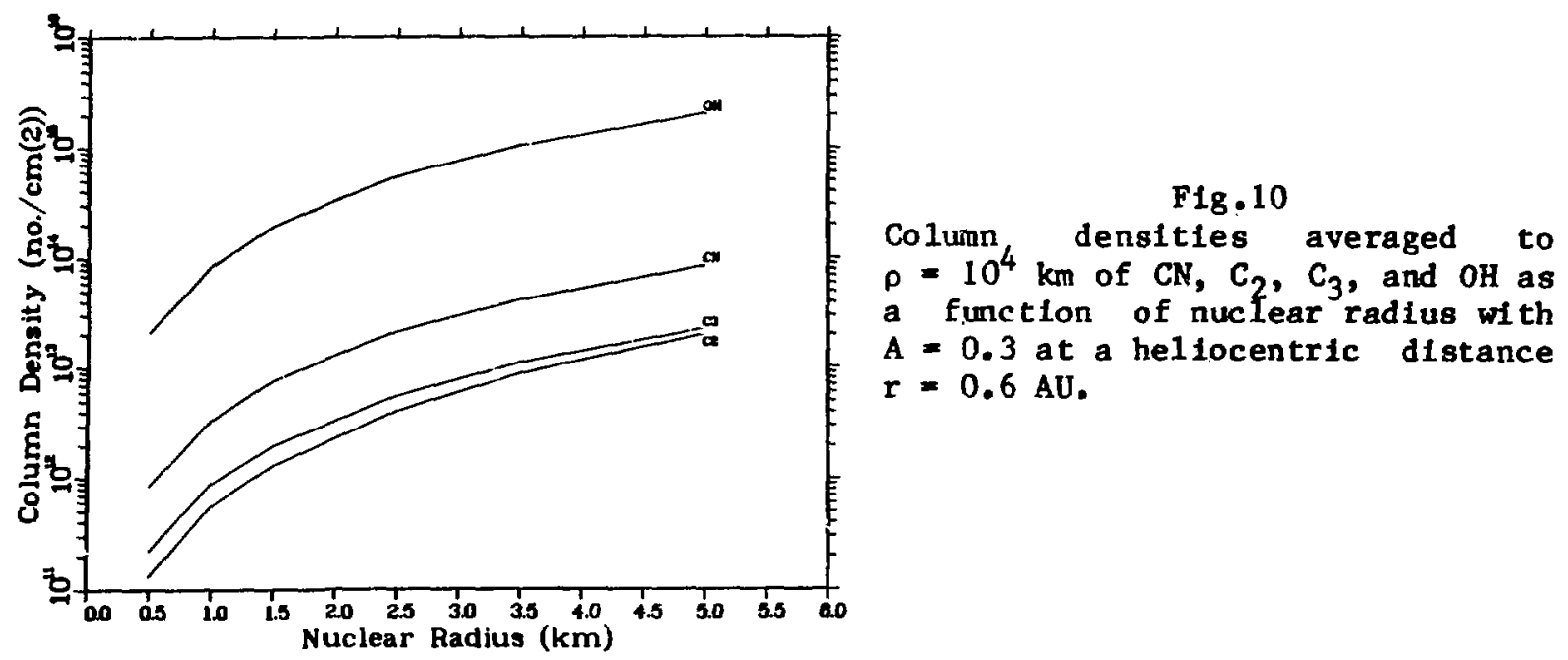

Fig.11

Column densities averaged to $\rho=10^{4} \mathrm{~km}$ of $\mathrm{H}_{2} \mathrm{O}^{+}$and $\mathrm{CO}^{+}$as a function of hellocentric distance for three nuclear radil (with $A=0.3)$ : dotted 1 ine $R_{0}=5.0 \mathrm{~km}$, solid IIne $R_{0}=2.5 \mathrm{~km}$, and chain-dashed 1 ine $R_{0}=0.5 \mathrm{~km}$.
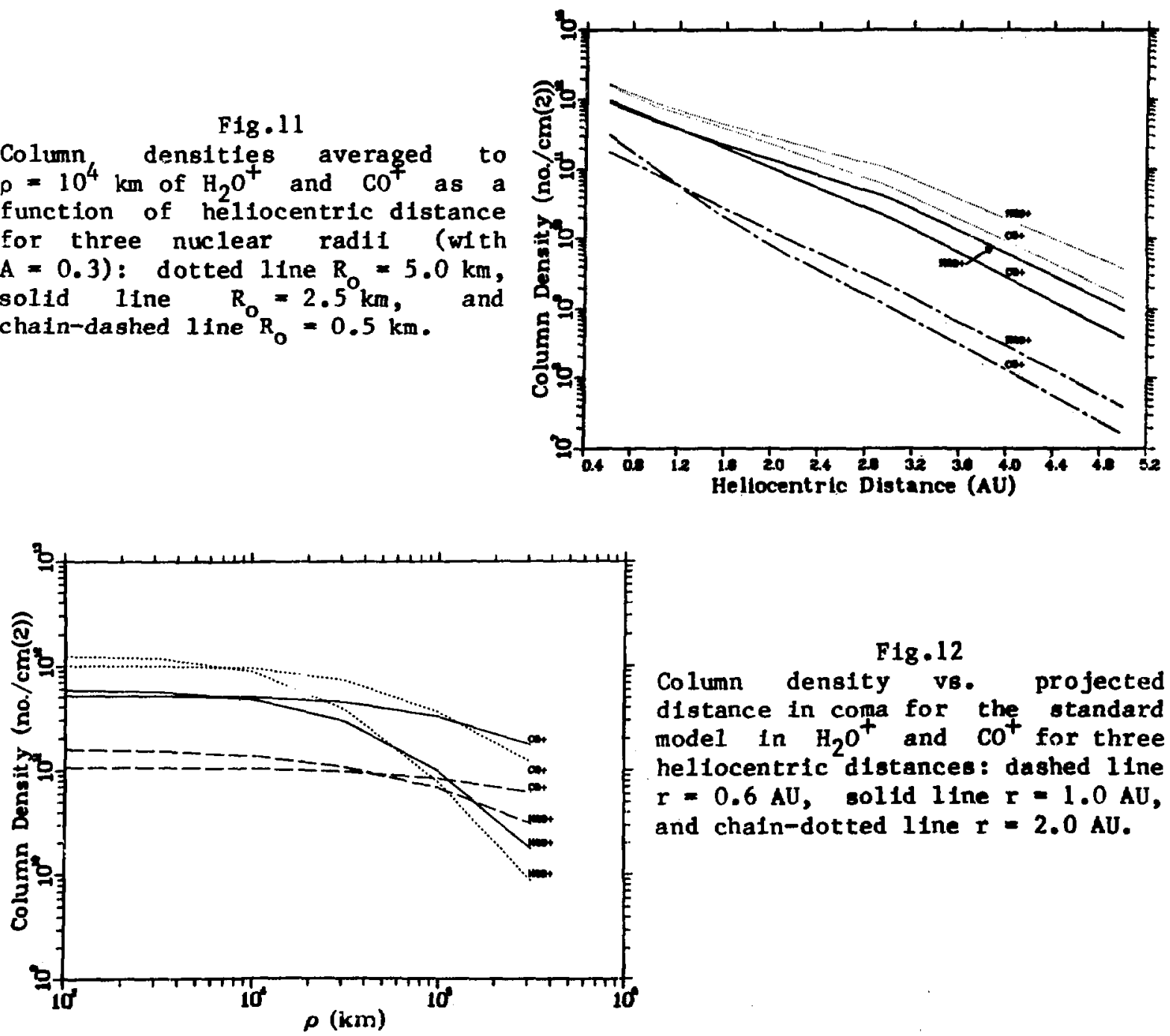

Fig.12

Column density vB. projected distance in coma for the standard model in $\mathrm{H}_{2} \mathrm{O}^{+}$and $\mathrm{CO}^{+}$for three hellocentric distances: dashed I1ne $r=0.6 \mathrm{AU}$, solid line $r=1.0 \mathrm{AU}$, and chaln-dotted line $r=2.0 \mathrm{AU}$. 


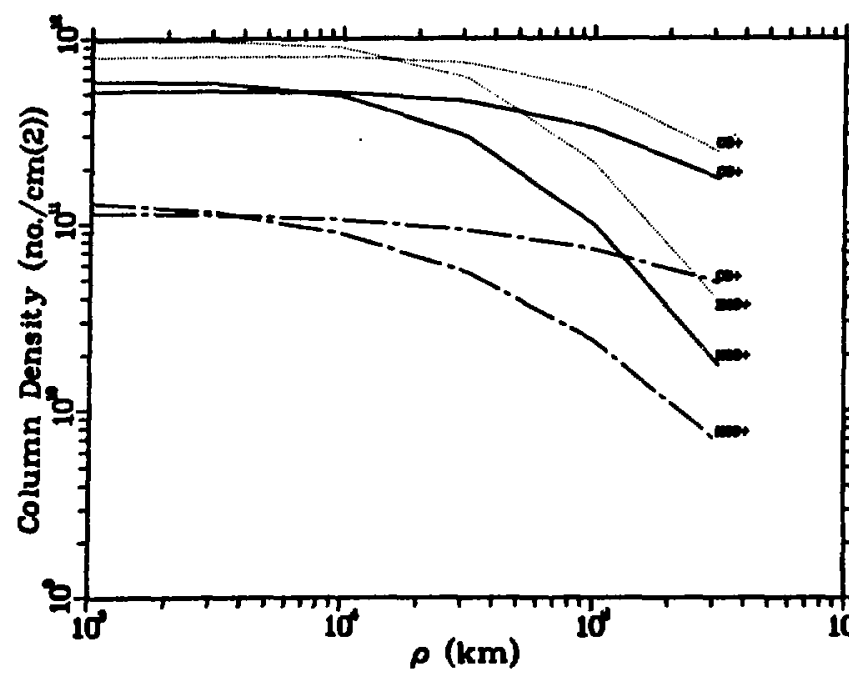

F1g.13

Column density vs. projected distance in coma for the lons $\mathrm{H}_{2} \mathrm{O}^{+}$ and $\mathrm{CO}^{+}$at $\mathrm{r}=1.0 \mathrm{AU}$ heliocentric distance with $A=0.3$ for three nuclear radil: dotted line $R_{0}=5.0 \mathrm{~km}$, solid line $R_{0}^{0}=2.5 \mathrm{~km}$, and chain-dashed line $R_{0}^{0}=0.5 \mathrm{~km}$.

$\mathrm{FIg} \cdot 14$

Column density vs. albedo $A$ for a nucleus with radius $R_{0}=2.5 \mathrm{~km}$ at $r=1.0 \mathrm{AU}$ heliocentric distance. column densities averaged to $\rho=10^{4} \mathrm{~km}$.
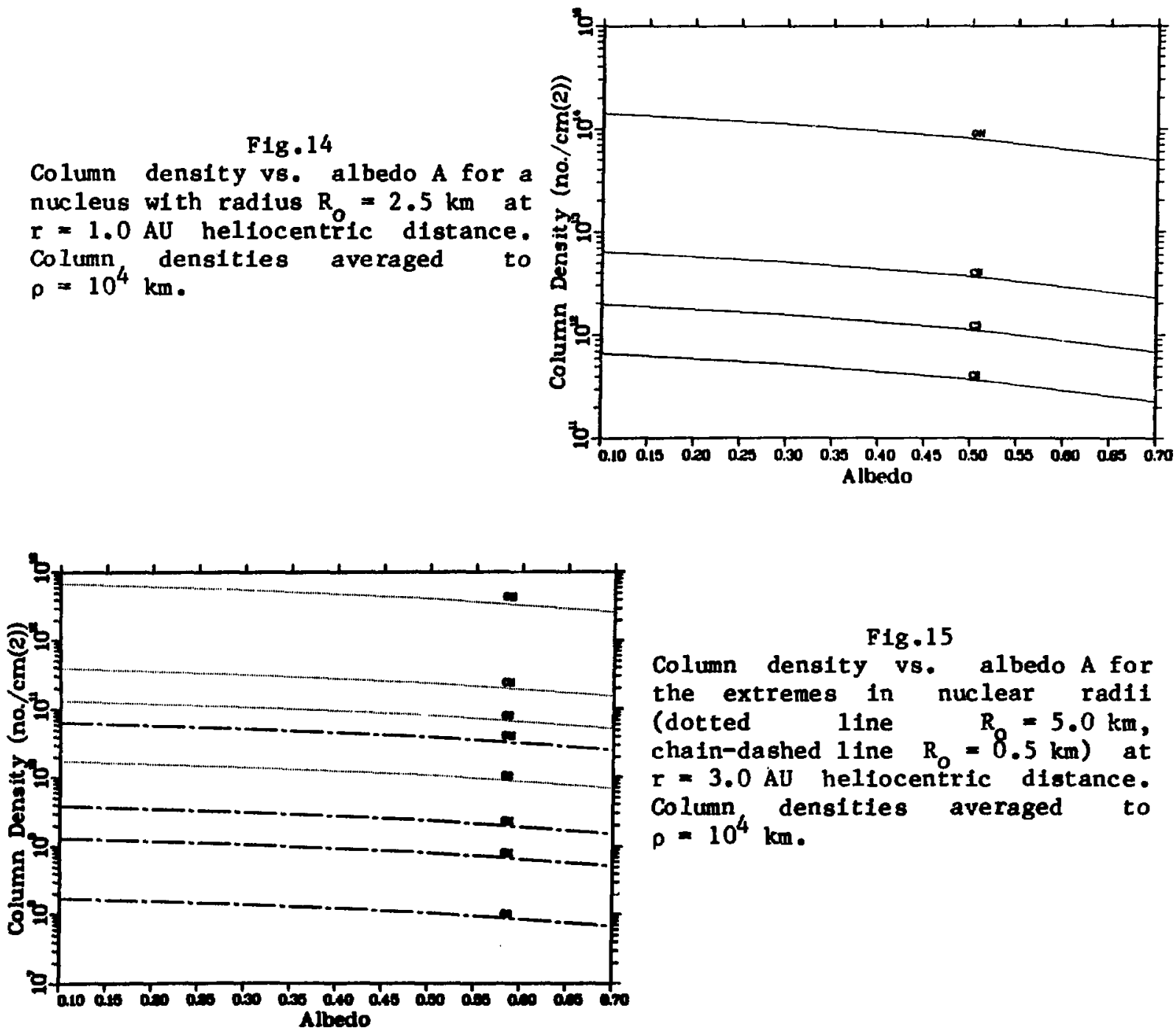

F1g.15

Column density vs. albedo A for the extremes in nuclear radi1 (dotted line $R_{0}=5.0 \mathrm{~km}$, chain-dashed line $R_{o}=8.5 \mathrm{~km}$ ) at $r=3.0 \mathrm{AU}$ hellocentric distance. column densities averaged to $p=10^{4} \mathrm{~km}$. 


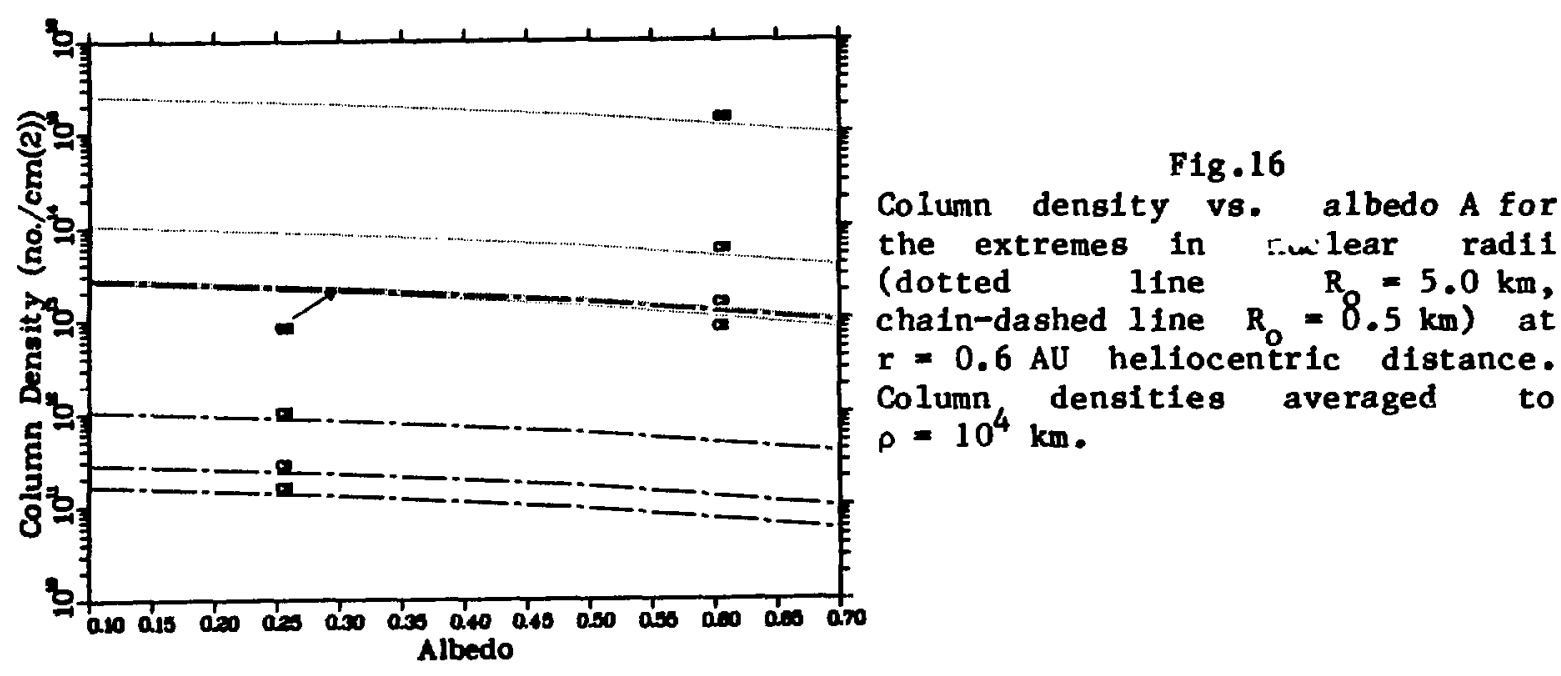

Fig. 17

Column density vs. nuclear radius $R_{0}$ for the extremes in albedo (solid Iine $A=0.7$, chain-dotted line $A=0.1$ at $r=3.0 \mathrm{AU}$ hellocentric distance. Column densities averaged to $p=10^{4} \mathrm{~km}$.

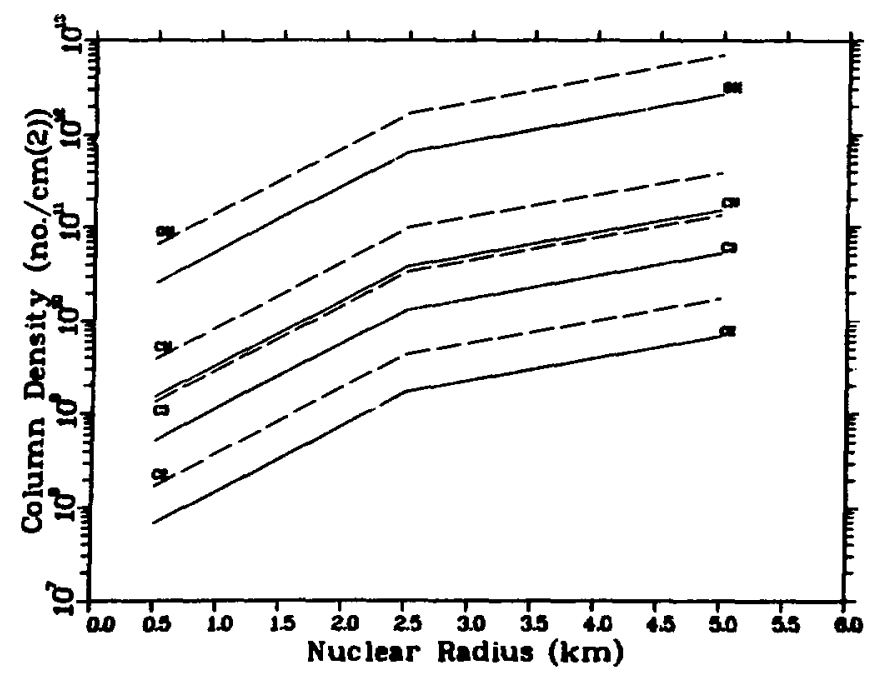




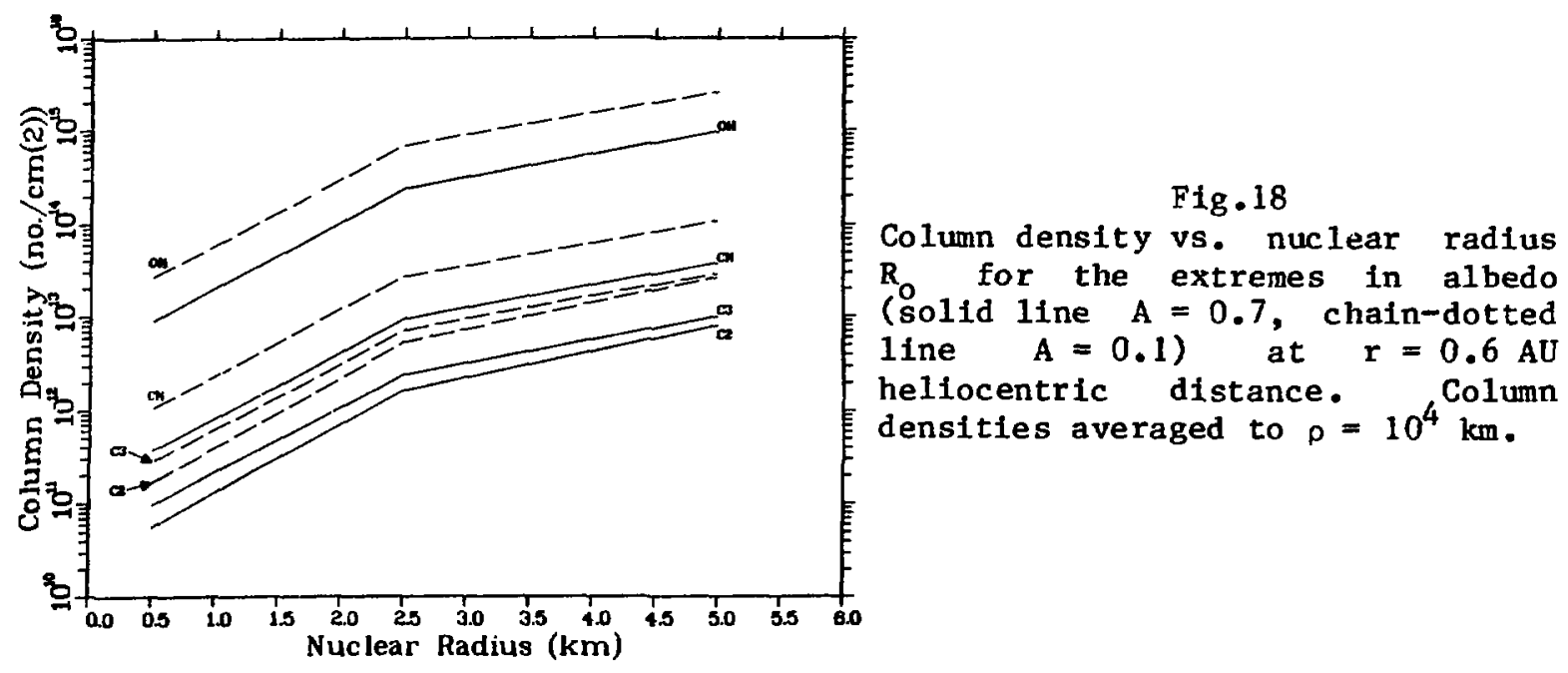

Fig. 19

Column densities averaged to $\rho=10^{4} \mathrm{~km}$ of $\mathrm{CN}, \mathrm{C}, \mathrm{C}_{3}$, and $\mathrm{OH}$ as a function of hellocentric distance for the standard model composition $6 a^{\prime}$. For this, the initial abundance of $\mathrm{C}_{2} \mathrm{H}_{2}$ has been increased from $0.1 \%$ to $0.16 \%$ and $\mathrm{H}_{2} \mathrm{C}_{3} \mathrm{H}_{2}$ has been reduced from $0.2 \%$ to $0.005 \%$ (see Sec. II B). Compare with Fig.5 for the unmodified composition $6 \mathrm{a}$.

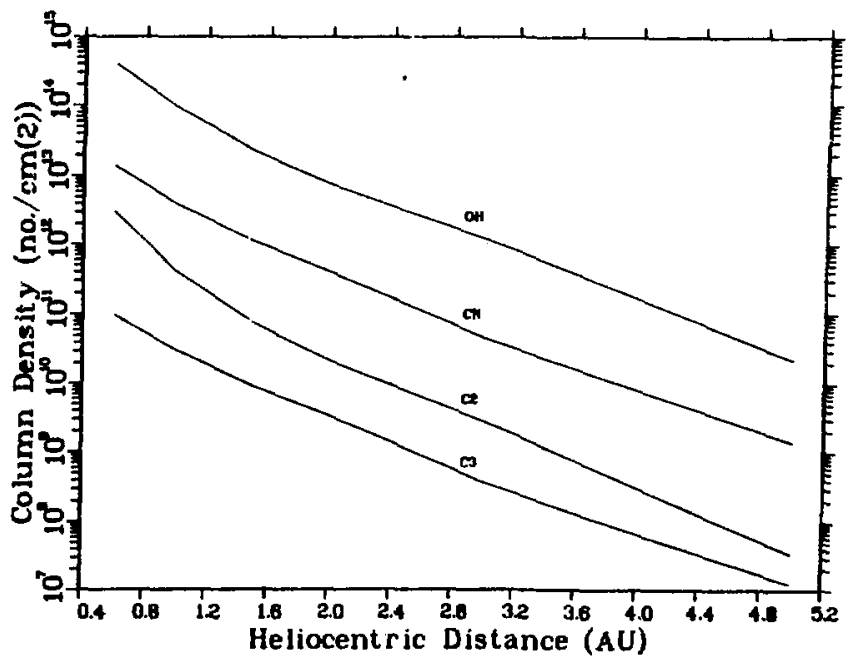

